\title{
MODELS FOR HETEROGENEOUS VARIABLE SELECTION
}

\section{DISSERTATION}

\author{
Presented in Partial Fulfillment of the Requirements for \\ The Degree Doctor of Philosophy in the Graduate \\ School of The Ohio State University
}

\section{By}

Timothy J. Gilbride, M.B.A., M.A

$* * * * *$

The Ohio State University

2004

Dissertation Committee:

Approved by:

Professor Greg M. Allenby

Advisor

Department of Business Administration

Professor Robert P. Leone

Professor H. Rao Unnava 



\begin{abstract}
Marketing managers are interested in knowing how consumers will react to different product configurations. The product manager can change physical attributes through the design of the product and the perception of psychological attributes through promotion strategies. Because consumers are heterogeneous in their tastes and preferences, aggregate level estimates of attribute importance are insufficient to describe the market. New research methods focus on obtaining individual level estimates of attribute importance from a representative sample of consumers.

Marketing researchers have procedural and statistical methods of obtaining measures of attribute importance for each respondent on each attribute. In laboratory or experimental choice settings, studies can be designed to help focus respondents' attention and processing of the product attributes. Bayesian methods of modeling heterogeneity shrink poorly measured individual level parameters to the overall or group level mean. However, it is erroneous to assume that consumers use all the product attributes in all brand choice situations. This thesis demonstrates that improved inference and predictive accuracy can be obtained by modeling which attributes are actually being used by consumers in different discrete choice situations.

This thesis contributes new models for determining, at the individual level, which product attributes are being used by a consumer in a brand choice decision. The
\end{abstract}


heterogeneous variable selection model extends current aggregate level models of Bayesian variable selection. This model assumes a distribution of heterogeneity with mass concentrated at 0 and away from 0 for each parameter. The pooled variable selection model allows the set of attributes used by an individual to vary by choice context. Examples of separate contexts include partial and full profile choice experiments or choice experiments and actual market place transactions. A hybrid model combines the heterogeneous and pooled variable selection models. The threshold variable selection model incorporates insights from an extended model of choice and provides a behavioral explanation of why certain product attributes are used. Tractable algorithms are introduced for estimating the proposed variable selection models. In the two empirical studies presented, a variable selection model fits the data better than baseline models with no variable selection and conventional distributions of heterogeneity. 
Dedicated to my wife, Teresa Minardi Gilbride 


\section{ACKNOWLEDGMENTS}

I wish to thank my advisor, Greg M. Allenby, for the time and effort he put into my doctoral education. I have benefited academically, professionally, and personally from my association with Greg. The other members of my committee, Robert P. Leone and H. Rao Unnava, not only provided guidance and support during my dissertation research but throughout my time at Ohio State. I want to thank the other members of the marketing faculty, especially James L. Ginter, Thomas Otter, and Patricia M. West, for their contribution to my doctoral studies. I want to thank current and past Ph.D. students particularly Yancy Edwards, Ling-Jing Kao, Jaehwan Kim, Kyeong Sam Min, and Priyali

Rajagopal. Cindy Coykendale provided invaluable help on navigating all manner of administrative details while I was at Ohio State. I also want to thank John Rapp of the University of Dayton for the instrumental role he played in leading me to "labor in the vineyards of higher education."

I could not have started or completed my doctoral studies without the support of my family and friends. My wife Teresa sacrificed much and shouldered an enormous burden while I was in graduate school. I am unable to articulate the magnitude of her contribution or the depth of my gratitude. My children, Harrison, Helen, and Hope are a constant source of encouragement, joy, and inspiration. They also made numerous 
sacrifices. My parents, Jerome and Virginia Gilbride, have always supported me and set the example by which I try to live. My father-in-law John Minardi not only offered encouragement but also commiserated on programming in Fortran 77. Finally, I am grateful to my extended family of Gilbrides and Minardis who are too numerous to list.

I chose to pursue doctoral studies to try and create value and serve others through my research and teaching, and ultimately, to strive to be a better person. I hope that my work is worthy of the investment of time and effort of my professors. I pray that my conduct is worthy of the love and support I received from my family and friends. 


\section{VITA}

July 28, 1966

1988

1993

2003
Born - Medina, OH, USA

B.S.B.A., The University of Dayton Economics, Dayton, OH, USA

M.B.A., The Ohio State University, Columbus, OH, USA

M.A., The Ohio State University, Business Administration, Columbus, OH, USA

Graduate Teaching and Research Associate, The Ohio State University

\section{FIELD OF STUDY}

Major Field: Business Administration 


\section{TABLE OF CONTENTS}

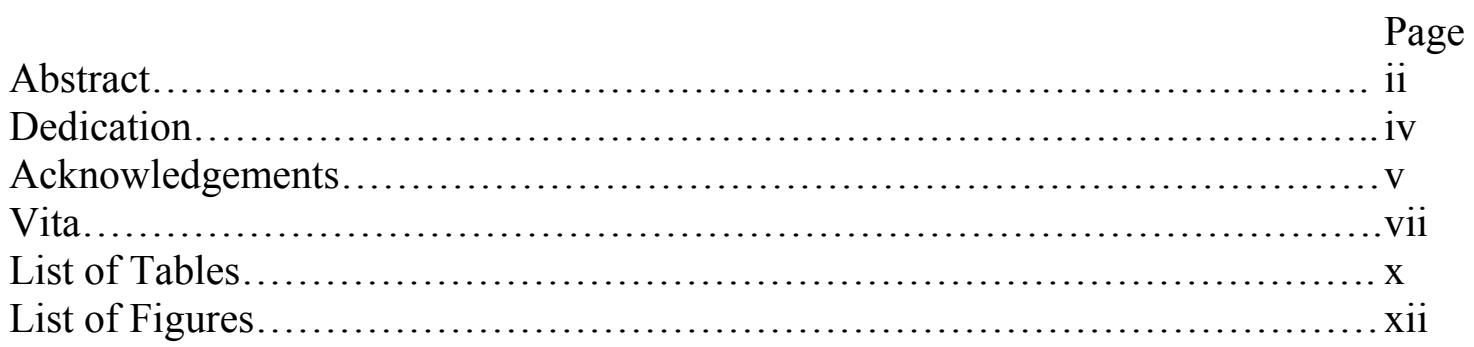

\section{Chapters:}

1. Introduction............................................................ 1

2. Literature Review................................................. 7

2.1 Bayesian variable selection....................................... 8

2.2 Three perspectives on extended models of choice....................... 13

2.2.1 Fennell's model of action........................................ 13

2.2.2 McFadden/Ben-Akiva extended framework for modeling choice.....17

2.2.3 Bagozzi's action theory model of consumption.................... 20

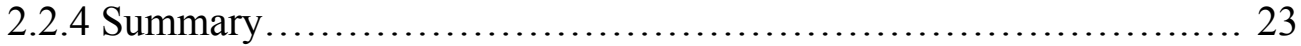

3. The Models............................................................ 32

3.1 Heterogeneous variable selection model f............................. 33

3.1.1 Model derivation ........................................... 34

3.1.2 Estimation algorithm........................................ 36

3.1 .3 Simulation results......................................... 40

3.2 Pooled variable selection model...................................... 41

3.2.1 Model derivation ............................................. 42

3.2.2 Estimation algorithm....................................... 44

3.2.3 Simulation results........................................... 46 
3.3 Hybrid model........................................................ 47

3.3.1 Model derivation ............................................. 48

3.3.2 Estimation algorithm........................................ 50

3.3.3 Simulation results.......................................... 53

3.4 Threshold variable selection model.................................... 54

3.4.1 Model derivation ................................................. 54

3.4.2 Estimation algorithm............................................ 61

3.4.3 Simulation results.............................................. 64

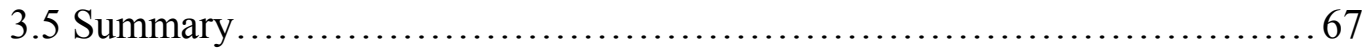

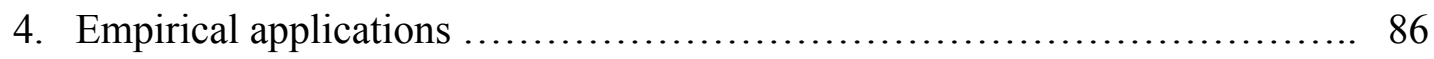

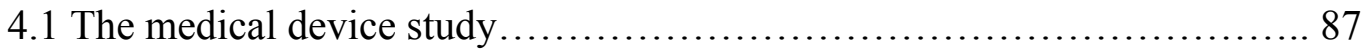

4.1.1 Data and models ................................................ 87

4.1.2 Results...................................................... 90

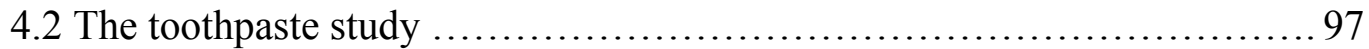

4.2.1 Data and models................................................ 97

4.2.2 Results...................................................... 101

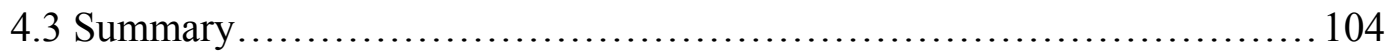

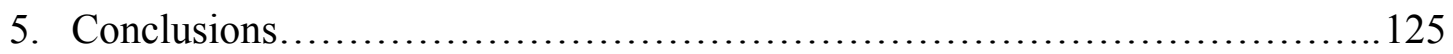

Appendix A: Derivation of conditional probability for the hybrid model ........... 130

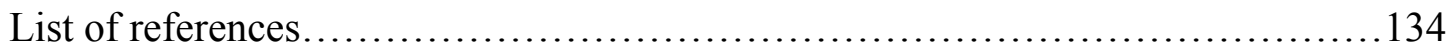




\section{LIST OF TABLES}

Table

Page

3.1 Simulation results for heterogeneous variable selection model compared to standard model

3.2 Simulation results for the pooled variable selection model

3.3 Simulation results from the hybrid variable selection model

3.4 Simulation results for the threshold selection model, diagonal: one-to-one mapping, multiple observations

3.5 Simulation results for the threshold variable selection model, row: one-to-many mapping, multiple observations.

3.6 Simulation results for the threshold variable selection model, column: many-to-one mapping, multiple observations....

3.7 Simulation results for threshold variable selection, diagonal: one-to-one mapping, single observation per respondent, homogeneous scale use.

3.8 Simulation results for threshold variable selection model, row:one-to-many mapping, single observation per respondent, homogeneous scale use...

3.9 Simulation results for threshold variable selection model, column: many-to-one mapping, single observation per respondent, homogeneous scale use.

3.10 Simulation results for threshold variable selection model, diagonal: one-to-one mapping, single observation per respondent, heterogeneous scale use

3.11 Simulation results for the structured variable selection model, row: one-to-many mapping, single observation per respondent, heterogeneous scale use. 
3.12 Simulation results for the threshold variable selection model, column: many-to-one mapping, single observation per respondent, heterogeneous scale use...

4.1 Comparison of model fit and predictive results for the medical device study... 106

4.2 Comparison of $\bar{\beta}$ estimates for the medical device study.

4.3 Posterior estimate of covariance matrix for the medical device study, pooled variable selection model.

4.4 Posterior estimate of covariance matrix for the medical device study, baseline model - all attributes included.

4.5 Comparison of attributes selected: medical device study.

4.6 Comparison of model fit for the toothpaste study

4.7 Comparison of part-worth estimates for the toothpaste study.

4.8 Comparison of part-worth estimates for the toothpaste study, sorted by relative size.

4.9 Posterior estimate of the covariance matrix for threshold variable selection model, column: many-to-one mapping with homogeneous scale use

4.10 Estimates of $\theta$ : Average mapping between concerns/interests and attributes/benefits for threshold selection model, column: many-to-one mapping, with homogeneous scale use.

4.11 Summary of attributes used, sorted by posterior mean for the threshold variable selection model, column: many-to-one mapping, with homogeneous scale use.

4.12 Summary of unmet concerns/interests, sorted by posterior mean for the threshold variable selection model, column: many-to-one mapping, with homogeneous scale use. 


\section{LIST OF FIGURES}

Figure $\quad$ Page

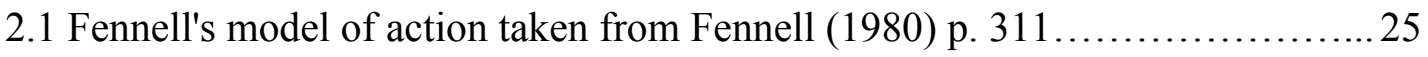

2.2 Fennell's model of brand choice taken from Fennell (1985) pp. 122-123 ........26

2.3 McFadden's model of the choice process taken from McFadden (2001) p. 356..27

2.4 Empirical modeling approach of McFadden/Ben-Akiva taken

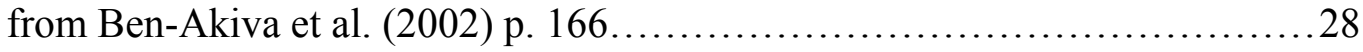

2.5 Bagozzi's action theory model of consumption taken from

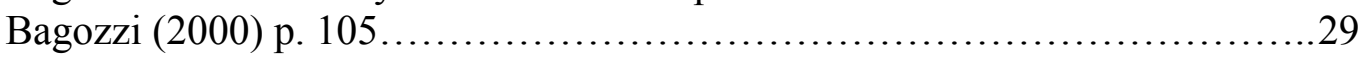

2.6 Bagozzi's model of goal directed behavior taken from

Bagozzi, Gurhan-Canli, and Priester (2002) p. 95 .............................30

2.7 Typical LISREL approach of Bagozzi taken from

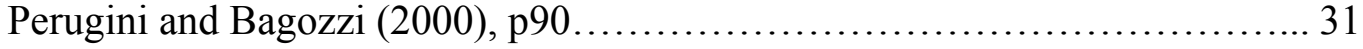

3.1 Comparison of models and substantive issues..............................69

3.2 Distributions of heterogeneity with mass centered at 0 and away from $0 \ldots \ldots \ldots . .70$

3.3 Activated explanatory variables and the map to product attributes..............74

3.4 Different maps, $\Lambda_{\mathrm{h}}$, and vectors of indicator functions $\mathrm{I}\left(\gamma_{\mathrm{h}}>\alpha\right) \ldots \ldots \ldots \ldots \ldots \ldots .75$

3.5 Example showing that $\Lambda_{\mathrm{h}}$ and $\alpha_{\mathrm{h}}$ are not both well identified with one observed choice.................................................. 79

4.1 Attributes, attribute levels, and effects-leveling coding for the medical device study ........................................... 105

4.2 Comparison of selected attribute importance: medical device study............ 115 


\section{CHAPTER 1}

\section{INTRODUCTION}

Differences between consumers and the behavior of any one consumer across contexts have both quantitative and qualitative components. Many behavioral theories involve discrete, qualitative descriptions. For instance, in microeconomics, the choice probability of a good changes abruptly as its price moves from just below, to just above the budget constraint. Behavioral decision theory suggests that if a consumer knows that she will have to justify her choice to a third party, she may use a different decision strategy or focus on a different set of attributes than in a situation without the need to justify. Consumer heterogeneity may be characterized by different consumers using different subsets of product attributes in a brand choice decision. If managers are to use these insights, externally valid methods of measuring these qualitative differences and incorporating them into decision models must be developed.

This study develops statistical models for determining who is using which product attributes and when they use them. Using an extended model of choice, a structural model of why they use them is also proposed. Marketing researchers have used ratings data, conjoint analysis, and various models to infer which product attributes are important. Heterogeneity has been incorporated into these models to identify not only which attributes are important, but also who finds them important. This thesis incorporates 
qualitative differences between consumers and with-in consumers through both the distribution of heterogeneity and structural relationships. It extends the notion of heterogeneity into the realm of qualitative differences where some attributes are important and used in the product choice decision, and others are unimportant and not used. Structural relationships include "regime" shifts across contexts and discrete relationships between explanatory variables and product attributes.

Identifying which attributes are used in a brand choice decision is closely related to the statistical procedure of variable selection. In many statistical analyses there are a large number of potential predictor variables and there is uncertainty about which variables are redundant or irrelevant. Variable selection seeks to identify the best, or most promising subset of variables to include in the model. In recent years there has been a large literature on Bayesian methods of variable selection and the related topic of model averaging. The statistics literature has focused on variable selection at the aggregate level; e.g. selecting the best subset of variables for the whole sample under study.

Research in marketing has focused on explicitly modeling heterogeneity. In marketing data sets we frequently have multiple data points per person in the form of purchase history or multiple observations from choice experiments (e.g. conjoint). The heterogeneous variable selection model proposed in this thesis conducts variable selection at the individual level. As opposed to a continuous distribution of heterogeneity, this model uses a distribution of heterogeneity where each parameter comes from a distribution with mass concentrated at 0 and away from 0 . This represents 
the qualitative situation where different consumers use different subsets of product attributes.

Many studies in marketing collect data from several contexts. For instance, experimental choice data may be supplemented with data from actual market transactions for the same individual. Or a study may include both a partial profile and a full profile discrete choice exercise. As a consumer moves from one context to another, the product attributes used may change. The pooled variable selection model assumes consumers use all the product attributes in one context, but only a subset in the other context. This model pools the data across contexts and identifies for each individual the subset of variables used in the targeted setting. Here a "regime" shift or qualitative change occurs as the consumer moves from an experimental to an actual market setting; or from a situation where only some of the attributes are presented to a setting where all the product attributes are presented. A hybrid model is also proposed that incorporates features of both the heterogeneous variable selection model and the pooled variable selection model.

Extended models of choice provide a conceptual framework for determining which product attributes are important, who they are important to, and why they are important. Extended models of choice involve many variables and rich descriptions of the decision process. A challenge in estimating extended models of choice is that they frequently involve qualitative explanations that involve discontinuities with thresholds, screening rules, selection criteria, etc. Gilbride and Allenby (2003) estimate a model where consumers employ screening rules based on a subset of product attributes in a complex choice environment. In this study, a threshold variable selection model is proposed that relates the set of product attributes used in a brand choice decision to a set 
of explanatory variables. The model of action by Fennell (1985) is used as the conceptual framework for this extended model of choice.

The threshold variable selection model simultaneously represents an extended non-linear choice process and deals with the large number of variables needed to operationalize the theoretical model. Fennell's model of action implicates a large number of potential explanatory variables, referred to as concerns and interests. The model identifies which of these concerns and interests are activated, or exceed some threshold, and how the concerns and interests map to specific product attributes. This discrete mapping determines which attributes are selected and used in the brand choice decision. In this model specific attributes are important because they are responsive to an individual's concerns and interests.

This thesis contributes new models for performing variable selection at the individual level in discrete choice data. The heterogeneous variable selection model extends current aggregate level models of Bayesian variable selection. The pooled variable selection model allows the set of variables used by an individual to vary by choice context. A hybrid model combines the heterogeneous and pooled variable selection models. The threshold variable selection model incorporates insights from an extended model of choice and provides a behavioral explanation of why certain product attributes are used.

Bayesian methods are used to estimate these variable selection models. Hierarchical methods capture parameter heterogeneity and are instrumental in defining the Markov chain Monte Carlo procedures used to navigate the space of models and parameter values. The algorithms are reviewed in detail and are conceptually similar in 
spirit the to Stochastic Search Variable Selection (SSVS) procedure of George and McCulloch (1993).

Two empirical studies are presented. In the Medical Device Study, the heterogeneous variable selection model, pooled variable selection model, and the hybrid model are fit to the data. In the Toothpaste Study, the heterogeneous, pooled, and threshold variable selection models are estimated. In both studies, a variable selection model fits the data better than baseline models with no variable selection and conventional distributions of heterogeneity. The Medical Device Study has data available for hold-out prediction and the variable selection models offer a 7 to $16 \%$ improvement in predictive accuracy. The Medical Device Study also shows that ignoring variable selection leads to biased parameter estimates and different conclusions about the importance of specific product attributes. These differences would result in different optimal product designs. The Toothpaste Study highlights the usefulness of using an extended model of choice. Specifically, the model identifies specific concerns and interests among consumers that are not being met by current product offerings. These unmet concerns and interests represent opportunities to reposition specific brands and/or develop new product offerings.

This thesis will proceed as follows. Chapter 2 reviews literature on Bayesian variable selection and literature from marketing, economics, and psychology on extended models of choice. The review focuses on the extended choice models by Fennell, McFadden/Ben-Akiva, and Bagozzi. The variable selection models are presented in chapter 3. Each model is derived algebraically followed by details on the estimation 
algorithm and simulation results. Chapter 4 presents the two empirical applications of the models. The thesis concludes in chapter 5 with a summary of the models, the empirical results, and possible extensions of this work. 


\section{CHAPTER 2}

\section{LITERATURE REVIEW}

This chapter reviews two disparate streams of research. The first part of this chapter reviews the literature on Bayesian variable selection. In recent years research has focused on taking advantage of modern computing techniques to exploit the integrated approach of Bayesian decision making to the issues of model selection, parameter estimation, and inference. Variable selection is a specialized subset of the broader issue of model selection. This part of the chapter will focus on the general set-up of variable selection, implementation issues, and how this thesis builds upon and extends this stream of research.

In the second section, three different extended models of choice from the marketing, psychology, and economics literature are reviewed. Extended models of choice provide a basis for understanding not only how consumers make specific brand choice decisions, but also why particular processes or variables are used in a particular instance. One of these, Fennell's model of action is used as the basis for the threshold selection model proposed in chapter 3 and used in the second empirical application in chapter 4 . 


\subsection{Bayesian Variable Selection}

The literature on variable selection is vast and in recent years much of the work has focused on Bayesian methods. George (2000) provides a succinct synopsis of recent research in this area and a guide to the literature. In theory, the Bayesian approach offers an integrated and consistent framework for model selection, parameter estimation, and inference. This review focuses on the general model set-up, implementation issues, and how this thesis builds upon and extends this stream of research.

Variable selection is a variant of the more general problem of model selection. If models $M_{1}, \ldots, M_{K}$ are considered, then the posterior probability of model $M_{k}$ is given by:

$$
\operatorname{pr}\left(\mathrm{M}_{\mathrm{k}} \mid \mathrm{Y}\right)=\frac{\operatorname{pr}\left(\mathrm{Y} \mid \mathrm{M}_{k}\right) \operatorname{pr}\left(\mathrm{M}_{\mathrm{k}}\right)}{\sum_{l=1}^{K} \operatorname{pr}\left(\mathrm{Y} \mid \mathrm{M}_{1}\right) \operatorname{pr}\left(\mathrm{M}_{1}\right)}
$$

where Y represents the observed data and

$$
\operatorname{pr}\left(\mathrm{Y} \mid \mathrm{M}_{\mathrm{k}}\right)=\int \operatorname{pr}\left(\mathrm{Y} \mid \theta_{\mathrm{k}}, \mathrm{M}_{\mathrm{k}}\right) \operatorname{pr}\left(\theta_{\mathrm{k}} \mid \mathrm{M}_{\mathrm{k}}\right) d \theta_{\mathrm{k}}
$$

where $\theta_{k}$ is the parameter vector associated with model $M_{k}, \operatorname{pr}\left(\theta_{k} \mid M_{k}\right)$ is the prior placed on $\theta_{\mathrm{k}}, \operatorname{pr}\left(\mathrm{Y} \mid \theta_{\mathrm{k}}, \mathrm{M}_{\mathrm{k}}\right)$ is the conditional likelihood of the data, and $\operatorname{pr}\left(\mathrm{M}_{\mathrm{k}}\right)$ is the prior probability of the model. In variable selection the class of models is typically limited to a common functional form (e.g. linear regression, multinomial logit, etc.) and $\mathrm{M}_{\mathrm{k}}$ is replaced by $\gamma_{\mathrm{k}}$ a vector that indexes the set of selected predictor variables. The object of posterior inference is therefore $\operatorname{pr}\left(\gamma_{\mathrm{k}} \mid \mathrm{Y}\right)$. Analyses that integrate over the model space $\mathrm{M}_{\mathrm{k}}$, or the space of all possible combinations of predictor variables $\gamma_{\mathrm{k}}$ to make inferences about parameters or predictive quantities of interest, are know as model averaging. 
A primary goal of variable selection algorithms is to avoid the enumeration and testing of all possible combinations of variables. If the number of potential variables equals $p$, then the formal evaluation of (1) and (2) would involve $2^{p}$ separate analyses; for a problem with 30 potential variables, this involves over a billion computations of the posterior probability. George and McCulloch $(1993,1997)$ have proposed a Bayesian approach referred to as Stochastic Search Variable Selection (SVSS) that makes use of the Gibbs sampler to simultaneously search over the parameter space and the set of included variables. As opposed to computing the entire posterior distribution of all models, the Gibbs sampler is used to identify promising subsets of models.

SVSS sets-up an irreducible Markov Chain with the posterior density of variables included in the model and parameter estimates as the target density. This is accomplished through the specification of the prior distribution. Let the vector of parameters be represented by $\beta$ and assume the prior is specified as $\operatorname{MVN}\left(0, D_{\gamma} R D_{\gamma}\right)$. R is the correlation matrix and $\mathrm{D}_{\gamma}$ is a diagonal matrix with elements equal to 1 or some small constant; the jj'th element of $D_{\gamma}$ equals 1 if $\gamma_{j}=1$ and the $j$ 'th variable is included in the model, otherwise the $\mathrm{jj}$ 'th element equals some small constant $=\mathrm{c}$. Under this set-up, $\mathrm{D}_{\gamma}$ determines the variable selection process while $\mathrm{R}$ contains any substantive assumptions about the a priori magnitude of the variance or relationship between the $\beta$ 's. If we assume a priori that the $\gamma_{\mathrm{j}}$ 's are independent and $\operatorname{pr}\left(\gamma_{\mathrm{j}}=1\right)=\mathrm{p}_{\mathrm{j}}$, then George and McCulloch (1993) demonstrate how a straightforward Gibbs sampler can be set-up for the case of aggregate level multiple regression. The multiple regression model is represented as: 


$$
\begin{aligned}
& \mathrm{Y} \mid \beta, \sigma^{2} \sim \operatorname{MVN}\left(\mathrm{X} \beta, \sigma^{2} \mathrm{I}\right) \\
& \beta \mid \gamma \quad \sim \operatorname{MVN}\left(0, \mathrm{D}_{\gamma} \mathrm{RD}_{\gamma}\right) \\
& \sigma^{2} \quad \sim \operatorname{IG}(\nu, V) \\
& \operatorname{pr}\left(\gamma_{\mathrm{j}}=1\right)=\mathrm{p}_{\mathrm{j}} \text { for } \mathrm{j}=1, \ldots, \mathrm{P}
\end{aligned}
$$

The Gibbs sampler simply entails successive draws from the full conditionals:

$$
\begin{aligned}
& \pi(\beta \mid \sigma, \gamma, Y) \\
& \pi(\sigma \mid \beta, \gamma, Y)=\pi(\sigma \mid \beta, Y) \\
& \pi\left(\gamma_{j} \mid \beta, \sigma, \gamma_{-j}, Y\right)=\pi\left(\gamma_{j} \mid \beta, \gamma_{-j}\right) \quad \text { for } j=1, \ldots, P
\end{aligned}
$$

where the notation $-\mathrm{j}$ indicates all other elements of the vector besides $\mathrm{j}$. As noted by George and McCulloch (1997), if $c=0$ then the chain (7) - (9) is irreducible; if $\gamma_{j}=0$, then the chain never leaves this state. George and McCulloch provide guidance on the choice of a "small constant" for use in $\mathrm{D}_{\gamma}$.

George and McCulloch (1997) extend this approach and provide analytical and computational improvements particularly in the conjugate case where $\beta$ is assumed a priori distributed $\mathrm{MVN}\left(0, \sigma^{2} \mathrm{D}_{\gamma} \mathrm{RD}_{\gamma}\right)$. In the conjugate linear regression case, it is straightforward to obtain:

$$
\pi(\gamma \mid \mathrm{Y})=\int \pi(\beta, \sigma, \gamma \mid \mathrm{Y}) d(\beta) d(\sigma)
$$

and alternative algorithms can be constructed to sample from $\mathrm{g}(\gamma) \propto \pi(\gamma \mid \mathrm{Y})$.

The George and McCulloch approach maintains a MVN prior distribution for all parameters. For some parameters, however, the density is very concentrated around 0 
(parameters which are not "included" in the model) and for others a rather diffuse prior is specified.

An alternative Bayesian approach is offered by Raftery, Madigan, and Hoeting (1997) which assumes that $\beta_{\mathrm{j}}=0$ with some probability otherwise $\beta_{\mathrm{j}}$ has some other appropriate distribution with mass away from zero. This prior distribution with a degenerate mass point at zero contrasts with the George and $\mathrm{McCulloch}$ approach where the prior distribution is specified as a mixture of two continuous distributions. Raftery, et al. propose a special MCMC sampler to deal with the discreteness of the prior distribution. For a given set of covariates included in a model at a particular point in the chain, a neighborhood is defined that includes one variable more, and one variable less than the current model. A Metropolis-Hastings type step is then used to choose the next model based on $\mathrm{g}(\gamma)$ as defined above. This method has also been used in Madigan and Raftery (1994) and other applications. A refinement on this approach, referred to as Occam's Window, restricts the set of neighborhoods to only those that are the most promising. Although the authors' admit the method is somewhat ad hoc, they have had success in several applications. For a comprehensive overview of these methods and applications, see Hoeting, Madigan, Raftery, and Volinsky (1999).

An important technical consideration in setting-up a MCMC method to do variable selection or model search is to ensure the chain is irreducible. As George and McCulloch (1997) state, the sampler must not get "stuck when it generates a value $\beta_{\mathrm{j}}=$ 0." George and McCulloch's algorithm achieves this through the use of a mixture of two proper priors and specifying $\mathrm{c} \neq 0$; Raftery, et. al. ensures the chain is irreducible via the definition of the "sampling neighborhood" and using $\mathrm{g}(\gamma)$ to choose the next set of $\gamma$. 
Geweke (1996) offers an algorithm wherein both the indicator and the parameters of interest $\left(\gamma_{j}, \beta_{\mathrm{j}}\right)$ are drawn jointly conditional on all the other parameters; this method also requires integrating out $\beta_{\mathrm{j}}$ from the appropriate posterior distribution. Green (1995) and Phillips and Smith (1995) offer theoretical representations and practical algorithms in the most general cases where proposed models may have different dimensions. In these cases it is generally necessary to calculate normalizing constants (e.g. use $\pi(\gamma \mid \mathrm{Y})$ and not $\mathrm{g}(\gamma)$ ) in assessing the probability of moving from one model to another in the sampler. Much of the recent literature in Bayesian variable selection and/or model averaging has focused on technical details associated with constructing efficient algorithms.

This thesis extends the previous literature by proposing variable selection models at the individual level. Much work has been done in marketing to explicitly account for heterogeneity across consumers (Allenby and Rossi, 1999). This thesis introduces methods to allow individuals to use different subsets of product attributes. Past research in variable selection has focused only on aggregate level analyses, e.g. selecting the best set of predictor variables for the entire sample under study. The variable selection models described in the next chapter are a new contribution to the literature.

The algorithms used to estimate the models are conceptually similar in spirit to the chain (7) - (9) and do not integrate out parameters and sample from $\pi(\gamma \mid \mathrm{Y})$. In this thesis, multinomial logit likelihoods at the individual level are paired with a multivariate normal distribution to capture heterogeneity; this is different than the conjugate models typically proposed in the statistics literature. Simply adding another layer to the hierarchy complicates the evaluation of $\pi(\gamma \mid \mathrm{Y})$ or $\mathrm{g}(\gamma)$. George and McCulloch's method 
of using an MCMC chain to explore the posterior space of both the models and model parameters forms the statistical underpinnings of the current approach.

Previous literature on variable selection used a completely data driven approach for identifying which variables to include in the model. By comparison, the proposed threshold selection model links antecedent conditions to the product attributes included in the brand choice decision. This thesis will use Fennell's extended model of choice to specify the relationship between antecedent conditions and product attributes. The next section compares three different extended models of choice from the marketing, psychology, and economics literature.

\subsection{Three Perspectives on Extended Models of Choice}

Although extended models of choice have been proposed in the marketing literature for over 35 years (Nicosia, 1964; Howard and Sheth, 1969; Engel, Kollat, and Blackwell, 1968, Bettman, 1979), this review focuses on three more recent models proposed by Fennell, McFadden/Ben-Akiva, and Bagozzi. The intent of this research is not to prove which model is correct. Rather, the primary focus of this thesis is how to connect the "boxes and arrows" of an extended model of choice/action in an empirical model. Of particular interest is how to algebraically specify the rich behavioral descriptions, e.g. the choice process.

\subsubsection{Fennell's Model of Action}

For Fennell, action is an individual's attempt to effect the specific counter-change needed to restore equilibrium in person-environment relations. An abbreviated version of 
the model of action based on Fennell (1980) is presented in Figure 2.1; a more detailed version can be found in Fennell (1988). Figure 2.2 presents a version of the model adapted for marketing from Fennell (1985). Characteristics of Fennell's approach are the attention given to the origin of a behavioral episode, identifying the source of heterogeneity in concerns and interests, the specificity of the ensuing process, and the availability of an applied (brand use/purchase) version of the general model of action for managerial use in meeting prospects' wants.

Action must be studied in the context of the relevant unit of analysis. A behavioral episode begins when the personal and environmental systems of an actor intersect, giving rise to the situation as perceived and calling attention to an imbalance between the current state and a desired state, in a particular substantive domain. An above threshold change in domain sensitivity allocates the individual's resources to effecting counterchange in that domain. An individual's personal sensitivities to physical and/or psychological phenomena as informed by his/her history, education, past experiences, and/or genetic make-up are implicated together with the environment to form the situation as perceived by the actor. The relevant unit of analysis is the person in an environment, or the situation as perceived by the actor, as opposed to the actor abstracted from the context and the particular substantive domain.

Heterogeneity is explicitly considered in the origins of the behavioral episode (Fennell 1980). Intersecting personal and environmental systems may take the form of one or more qualitatively different kinds of motivating conditions (Fennell 1978, see also Fennell and Allenby, 2003). There are five simple classes of motivating conditions: current problem, potential problem, normal depletion, interest opportunity, and sensory 
pleasure opportunity. Two complex classes, product-related problem and satisfactionfrustration, are used to represent situations where a consumer is already in the market for a particular product, but in addition to the five simple classes, another confounding element is present. Motivating conditions are operationalized as specific, but heterogeneous concerns and interests (c/i's). C/I's are conceptualized as either being "activated" for an individual and influencing the ensuing decision process or not being "activated". In actual studies, this can result in a large number of potential c/i's, only a subset of which may apply to an actor in a given context.

Fennell postulates a specific process leading to action. The activated c/i's give rise to a directed search for actions believed to be instrumental in bringing about the desired end-state. If the individual generates more than one, $\mathrm{s} / \mathrm{he}$ orders the candidate actions for instrumentality and, if the top candidate survives a test for costworthiness, the individual attempts to act. The action taken is, in the actor's view, the most suitable means of effecting counter-change. Following attempted action, the individual evaluates the outcome, and the behavioral episode closes with an updating of beliefs.

This model of action is applied to marketing when a brand is chosen or could be chosen to effect the necessary counter change and bring about the desired end-state. Figure 2.2 reflects substituting "behaviors \& stimuli" with "offerings" and the insertion of "Instrumental Attributes." "Instrumental Attributes" are the marketers' articulation of the "attributes of goods and services that will bring about customers' desired states." (Fennell, 1985 p.121) Producers must understand conditions as found upstream of market place offerings, in particular the nature of diverse motivations (e.g. concerns and interests) that give rise to the tasks and interests in an individual's life, in order to design goods and 
services that are responsive to those conditions. Producers do this by studying the $\mathrm{c} / \mathrm{i}$ 's with a view to understanding the kind of product attributes that would be instrumental for achieving the desired counter-change.

The threshold variable selection model, described in chapter 1 and in more detail in chapter 3, is an interpretation and application of Fennell's model of action. Product attributes are important, or desirable to the extent that they address the concerns and interests of a consumer in a particular consumption context. As contrasted with other extended models of choice, both $\mathrm{c} / \mathrm{i}$ 's and product attributes are conceptualized as "concrete" variables as opposed to abstract, or latent factors.

Fennell's model of action suggests several natural discontinuities in the decision process that are incorporated into the threshold variable selection model. First, particular concerns and interests are either "activated" and influence the decision process, or they are not. This implies that there is a threshold that must be identified in the model in order to determine which $\mathrm{c} / \mathrm{i}$ 's are influencing the decision process. In this thesis it is hypothesized that the mapping from an individual's c/i's to the corresponding desired product attributes is unique to the individual, discrete, and not necessarily complete (e.g. one may have a c/i that does not correspond to current marketplace offerings). Empirically estimating the map (representing the selection process) from c/i's to product attributes will be a primary contribution of this research. The model deals with the large number of variables used to operationalize the choice process by identifying on the individual level the activated c/i's and the subset of selected product attributes used for brand evaluation. 
The threshold variable selection model will add to and extend other empirical applications of Fennell's model of action. Yang, Allenby, and Fennell (2002) showed in a hierarchical Bayes model that brand preference is related to concerns and interests and product attributes in a regression type set-up. Fennell and Allenby (2003) study concerns and interests, attribute importance, and brand beliefs for toothpaste. Their results indicate that while there is a complex relationship between the three constructs, a simple model incorporating the relative importance of c/i's, attribute importance, and brand beliefs predicts actual brand choice better than a model with just attribute importance and brand beliefs, or a model with just c/i's and brand beliefs. Previous empirical applications have not attempted to model the discontinuities implied by Fennell's model of action that are included in the threshold variable selection model.

\subsubsection{McFadden/Ben-Akiva Extended Framework for Modeling Choice}

As contrasted with Fennell, the McFadden/Ben-Akiva conceptual framework incorporates a role for both concrete variables and abstract or latent factors. The choice process is represented by modifying the standard random utility model to incorporate these abstract factors. As such, the framework does not show why certain product attributes are valued, but does offer a method for incorporating motivating conditions into the final choice.

Figure 2.3 provides an overview of the extended model of choice from McFadden (2001). Other versions of this model have appeared in McFadden (1986), McFadden (1999), Ben-Akiva et al. (1999), and Ben-Akiva et al. (2002). The three boxes with the heavy borders represent the standard economic model of perception, process, and 
preference rationality. Boxes with lighter arrows represent the impact of psychological factors, the antecedent or motivating conditions of the choice process. As McFadden notes "The figure describes one decision-making task in a lifelong sequence, with earlier information and choices operating through experience and memory to provide context for the current decision problem, and the results of this choice feeding forward to influence future decision problems." (McFadden, 2001, p.355) The specific mechanics of how the psychological factors may influence the standard model are discussed in an enumeration of the empirical findings in the Behavioral Decision Theory (BDT) literature. The catalog of effects, while well documented, has not been unified into a single theory. Until "brain science understands how the cognitive mechanism operate" (ibid, p. 363), McFadden advocates modifying the standard random utility model to account for varying consumer perceptions. This approach is evident in the empirical applications of this framework.

Researchers operationalizing the McFadden/Ben-Akiva extended model of choice have focused on a generalized random utility framework estimated via simulated maximum likelihood. This modeling approach is represented graphically in Figure 2.4 (Ben-Akiva, et. al. 2002). At its heart is the standard random utility model. Virtually all applications of these models contain both revealed preferences (RP) from actual market behavior and stated preferences (SP) from a conjoint type of exercise. Heterogeneity and/or correlated error structures are introduced by including an additional random error term in the standard model. The additional error term may be constructed to represent heterogeneity in the parameters, referred to as a random coefficient model; or it may be structured to represent correlation between the choice alternatives, an error components 
model. Brownstone, Bunch and Train (1999) fit an error components model to SP data on choices between hypothetical alternative fueled vehicles and RP data on actual vehicle choice. Bhat and Castelar (2002) extend this model by incorporating both error components and random parameters in studying transportation mode choice.

Psychological variables representing differences in perceptions or attitudes are introduced as latent variables and included in the utility function (cf. Morikawa, BenAkiva, and McFadden, 2002). Additional structural and measurement equations are introduced to augment the standard random utility model. Each individual is hypothesized to have a unique value on one or more abstract factors such as "quality," "impatience," or "self-control." These latent variables may be related to either characteristics of the individual or the products under consideration in a separate structural equation. The latent variables are measured and identified in a factor analytic model of indicator variables, see Walker and Ben-Akiva (2002).

Simulated maximum likelihood is used to estimate the parameters in the model. Simulation methods are necessary since unobserved error terms and latent psychological factors must be integrated out of the likelihood function. Exploiting simulation methodology has allowed researchers to specify and estimate models with latent continuous variables used as explanatory variables.

There are several differences between the proposed threshold variable selection model and the generalized random utility approach used to operationalize the McFadden/Ben-Akiva extended model of choice. The generalized random utility model incorporates motivating conditions as "covariates" as opposed to the rather specific role played by concerns and interests in selecting desired product attributes in the threshold 
variable selection model. The generalized random utility approach is structurally unable to represent sharp behavioral hypotheses such as threshold values, attribute selection, or non-compensatory decision protocols suggested in either the BDT literature or in Fennell's model of action.

\subsubsection{Bagozzi's Action Theory Model of Consumption}

Bagozzi argues that economic models "leave out important mental phenomena essential to decision making in everyday consumption." (Bagozzi, 2000, p. 99). Bagozzi approaches action from a psychological paradigm and uses different theoretical constructs and empirical tools than either Fennell or McFadden/Ben-Akiva. In Bagozzi's framework, virtually all constructs are represented as latent psychological factors and a specific process is hypothesized. Abstract factors are identified through a factor analysis model and relationships between factors are represented as linear functions. Hence, LISREL has been the empirical technique used to operationalize this conceptual framework. Bagozzi's conceptual framework is outlined next.

Figure 2.5 represents an action theory model of consumption taken from Bagozzi (2000). A somewhat abbreviated version of this model, the model of goal directed behavior, from Bagozzi, Gurhan-Canli, and Priester (2002) is presented in Figure 2.6. Each of these models has the theory of reasoned action (Fishbein and Ajzen 1975) at its root and represents the evolution of the theory as new variables and processes have been identified and measured. These models are characterized by the role of goals, anticipated emotion, an extended process leading to action, and somatic marker processes. 
Goals are defined as a specific outcome or outcomes that consumption can produce. "A desired outcome enters the mind of the decision maker and can be defined as a specific type of goal ... Consumers make purchases to produce or yield one or more end-state goals." (Bagozzi and Dholakia, 1999, p.19). Studying goal directed behavior versus "just behavior" can be characterized by the difference in the following two statements: "I intend to do X in order to achieve Y" vs. " I intend to do X" (Perugini and Connor, 2000, p. 712). In order to understand consumer behavior, therefore, it is necessary to understand the goals a consumer is trying to achieve through his/her marketplace transaction. A goal may arise from either external stimuli (e.g. a change in the environment) or internal stimuli (e.g. the mere thought that the person has a need).

Anticipated emotions represent the decision maker's expectation of his/her affective state if the goal is achieved, or if it is not achieved. These represent the primary motives for acting and are viewed as dynamic, changing from "time to time, depending on the context" (Bagozzi et al. 2002, p. 94). In an empirical application, Perugini and Bagozzi (2001) measured 17 anticipated emotions: 7 positive and 10 negative that were considered indicators of 6 latent factors.

An extensive process leading to action and the role of subconscious mental processes are also incorporated into Bagozzi's action theory model of consumption. Action is ultimately the result of several steps: forming desires from attitudes, anticipated emotions, and subjective norms; forming intentions to act; trying; and ultimately acting. Along the way, frequency and recency of past behaviors, perceived behavioral control, and social identity may interact or moderate the process. Bagozzi hypothesizes that very early in the process, non-conscious biases restrict and limit the cognitive reasoning 
process pursued by the actor. These biases are the result of previous emotional experiences in similar decision problems and may highlight certain options and eliminate others from consideration at a subconscious level. Selecting only a subset of product attributes to use in the decision process may be a result of these non-conscious biases. This "somatic marker hypothesis" has support in neuroscience (Tranel, Bechara, and Damasio 2000) but has yet to be implemented in an empirical study in marketing.

Researchers implementing Bagozzi's extended model of action/choice have used LISREL to empirically operationalize and test models. Figure 2.7 represents the LISREL model used to test the model of goal directed behavior from Perugini and Bagozzi (2001). In separate investigations, Perugini and Bagozzi examined body weight regulation and study habits as the target behaviors; Perugini and Conner (2000) studied the same behaviors using a similar framework but did not study the actual performance of the behavior, only the volition to perform the behavior. Dholakia and Bagozzi (2002) looked at the role of decision process importance, decision process effort, and decision process confidence in influencing goal intention and goal realization. In this study, participants were asked to report on a non-routine purchase decision they encountered in their daily lives.

As compared to the generalized random utility model, the LISREL modeling approach represents all constructs as latent and can incorporate relationships between the motivating conditions and the desired product attributes. Depending on the constructs included and the specific model, empirical applications of LISREL can provide insight into why certain product attributes are valued. However, only particular interrelationships can be modeled; specifically, linear relationships with normally distributed 
errors. Heterogeneity is captured by different individual responses to the indicator variables used to measure the latent factors. The structural relationship is hypothesized to be the same across respondents. Both the proposed threshold variable selection model and the structural equation model incorporate relationships between motivating conditions and product attributes. However, the reliance of the structural equation or LISREL type of modeling on linear, compensatory relationships makes it inappropriate to represent behavioral models with discontinuities and/or thresholds.

\subsubsection{Summary}

Three different extended choice/models of action have been presented with different perspectives on how to represent the choice process and the reasons for action. In Fennell's model of action, product attributes are valued because they address specific c/i's of the actor in a particular context. Both motivating conditions and product attributes are represented as concrete variables and discontinuities and thresholds characterize the choice process. A new contribution to the literature, the threshold variable selection model, is proposed to operationalize Fennell's model. Empirical applications of the McFadden/Ben-Akiva extended choice framework do not structurally relate motivating conditions to desired product attributes, but instead include motivating conditions as abstract psychological factors in the utility function. In essence, choice is modeled controlling for the effect of motivating conditions. Bagozzi's conceptual framework is typically modeled empirically by a structural equation model where both motivating conditions and product attributes are conceptualized as abstract factors and interrelationships are represented as continuous linear functions. A discontinuous decision 
process and the representation of all variables as concrete, as opposed to latent factors, necessitates a new modeling framework which departs from existent generalized random utility and structural equation models.

This chapter has reviewed literature from statistics relating to Bayesian variable selection as well as literature from marketing, economics, and psychology on extended models of choice. In the next chapter, models are proposed which extend Bayesian variable selection to identify the best subset of variables at the individual level, incorporate contextual variation in the variables used, and to capture an extended model of choice that incorporates thresholds and discrete relationships to relate explanatory variables to the variables selected. 


\section{Fennell's model of action (1980)}

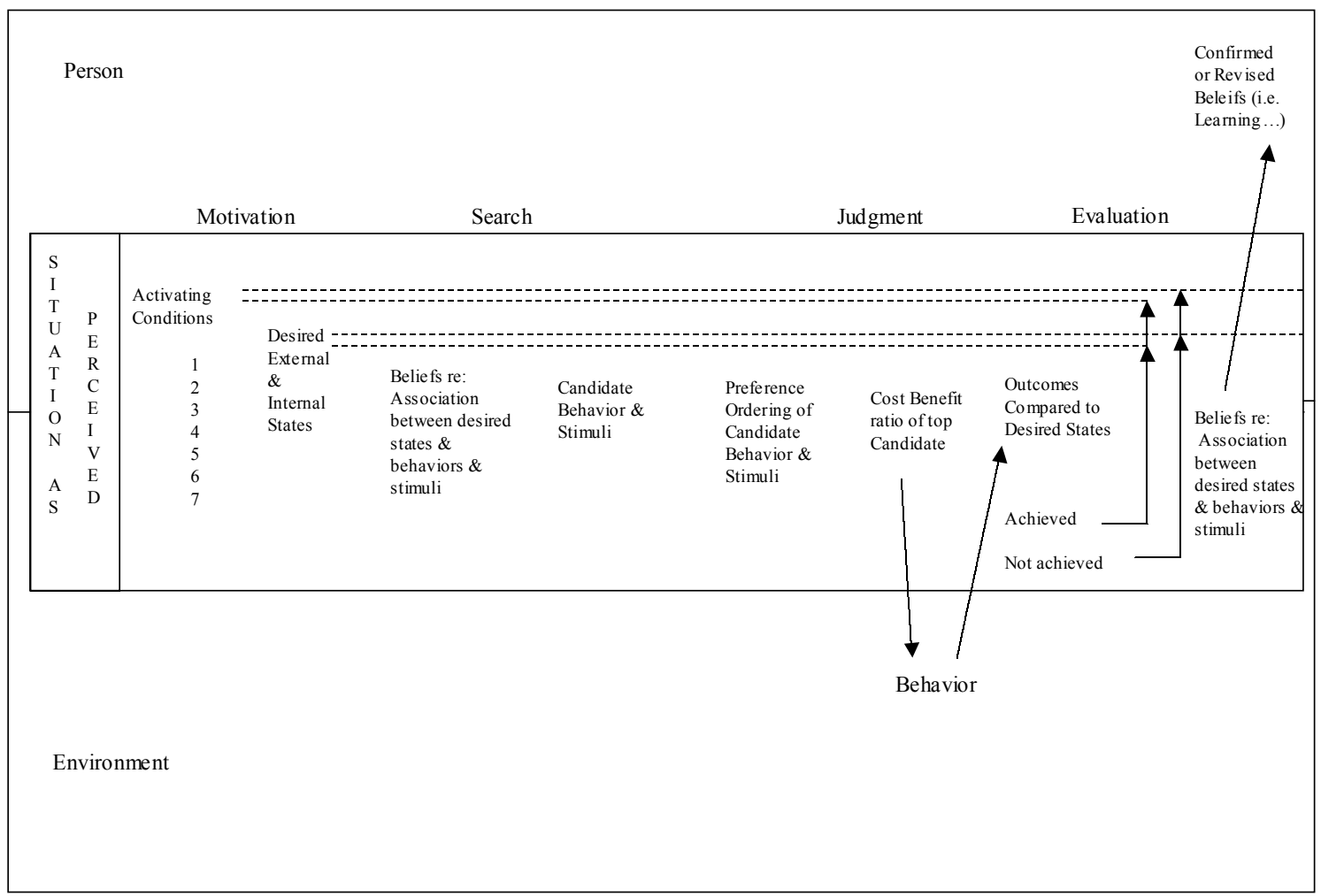

Figure 2.1 Fennell's model of action taken from Fennell (1980) p. 311. 


\section{Fennell's model of brand choice (1985)}

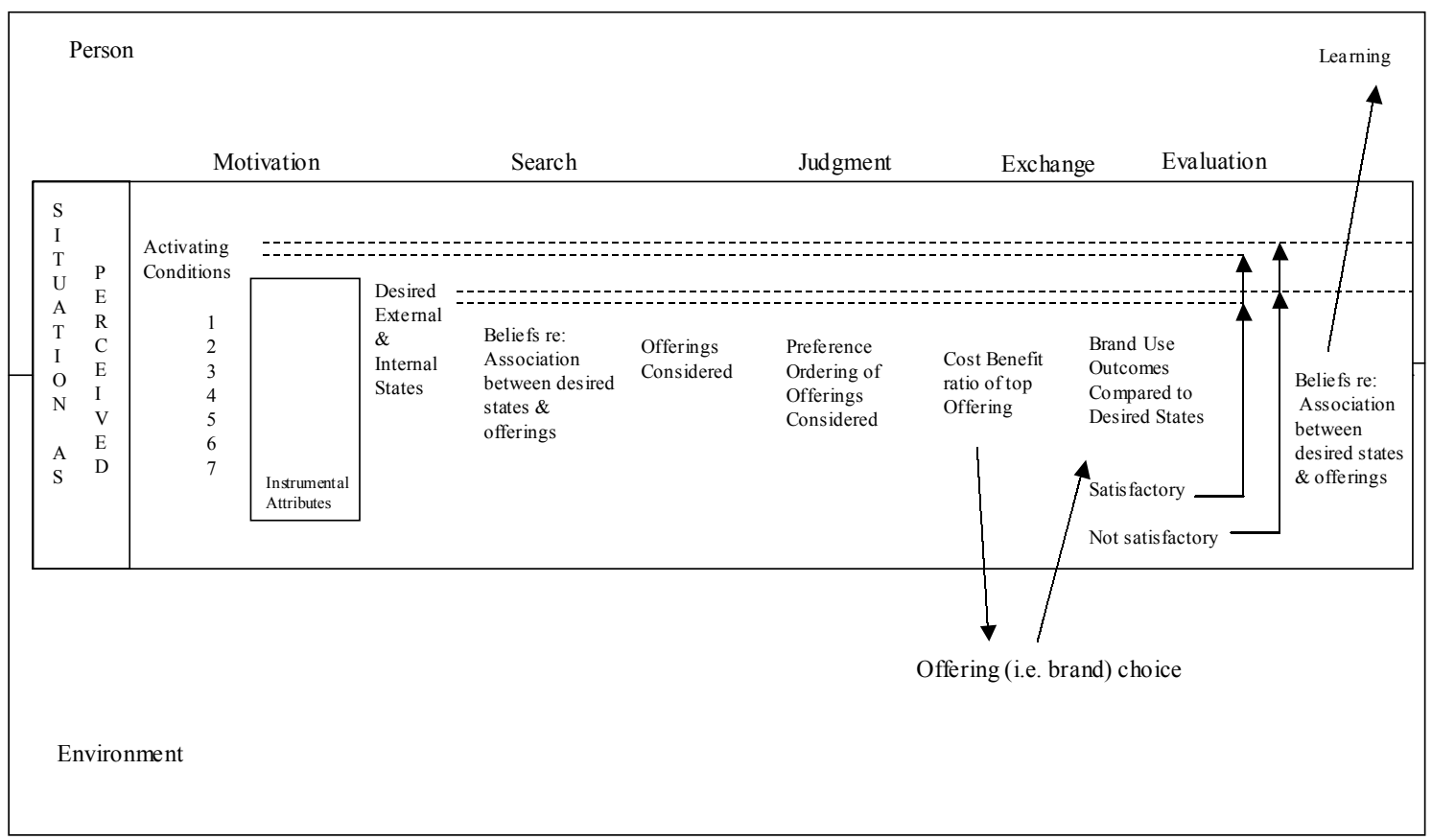

Figure 2.2 Fennell's model of brand choice taken from Fennell (1985) pp. 122-123. 
McFadden's model of the choice process (2001)

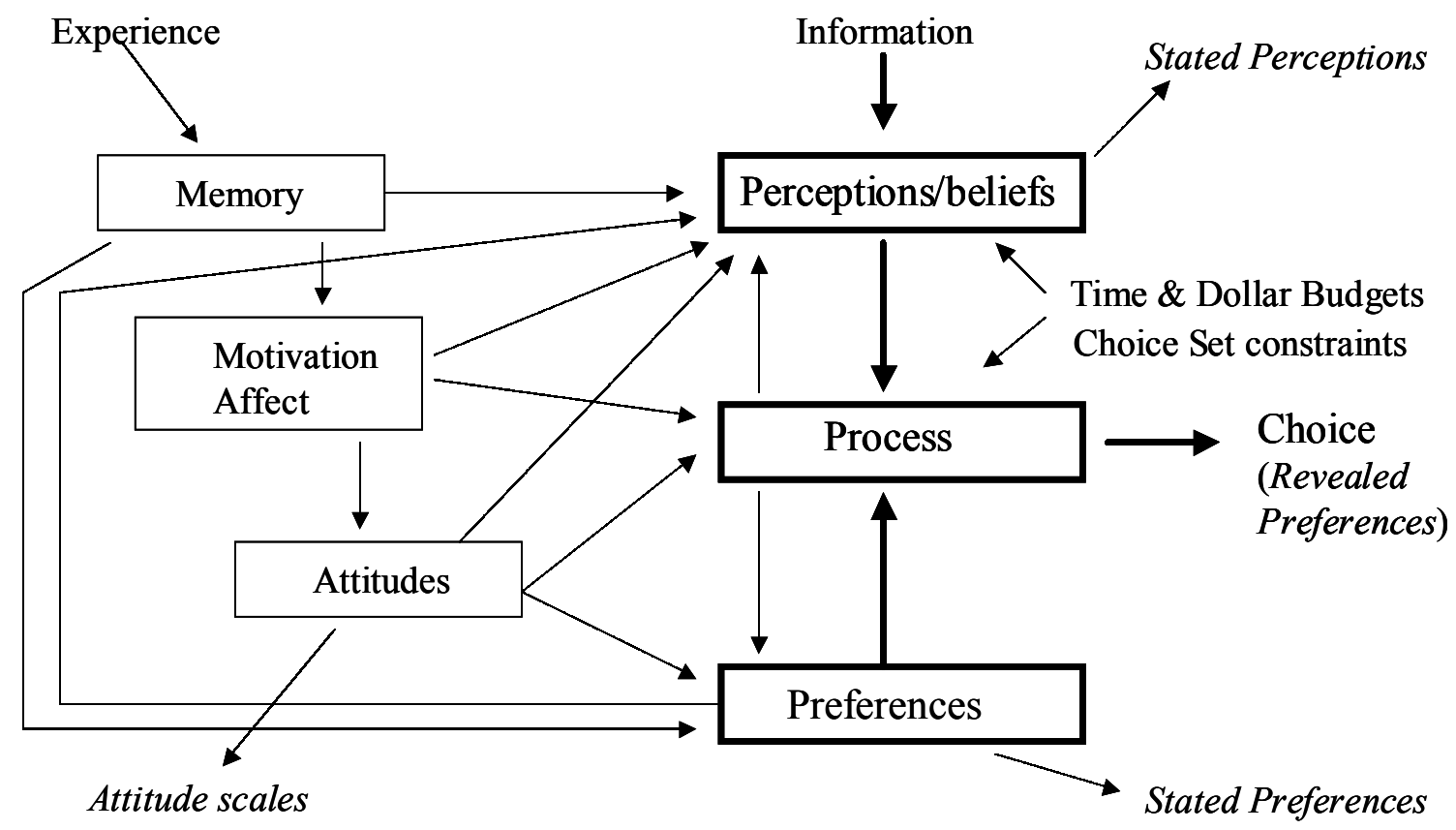

Figure 2.3 McFadden's model of the choice process taken from McFadden (2001) p. 356. 


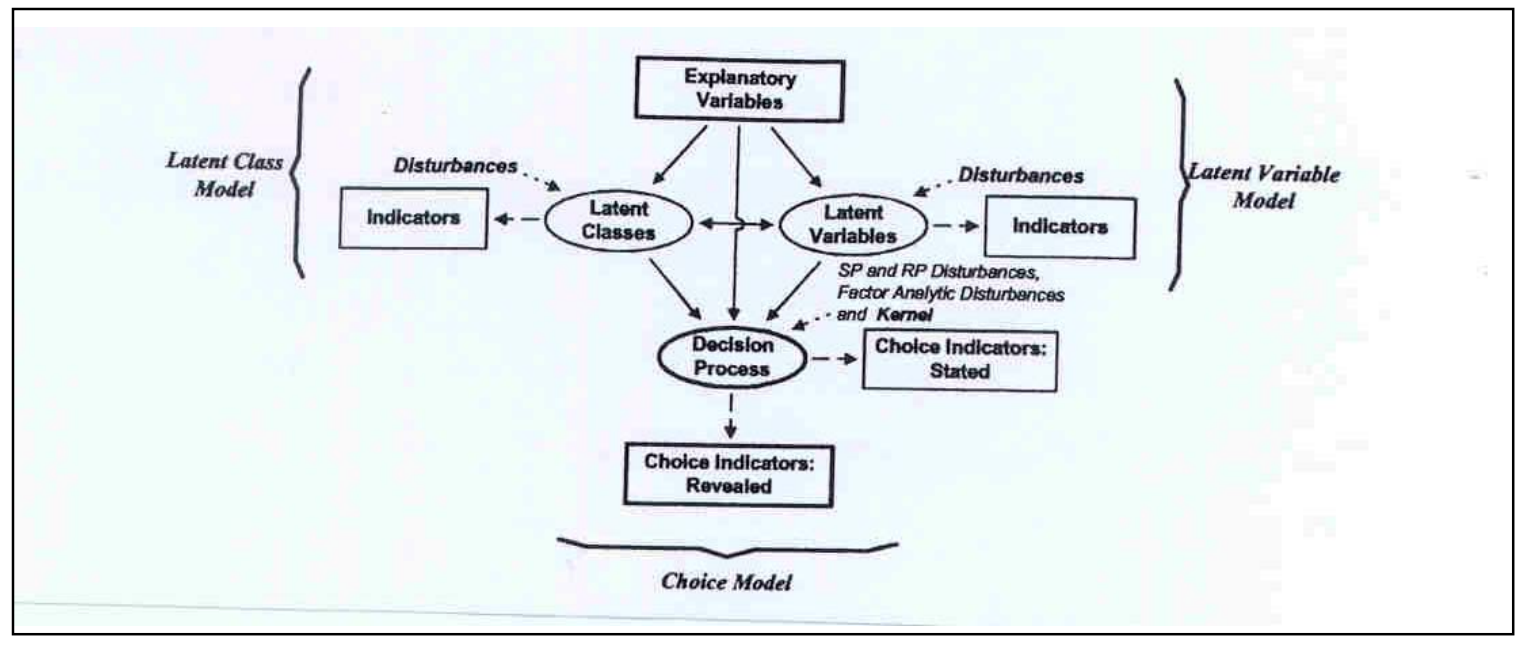

Figure 2.4 Empirical modeling approach of McFadden/Ben-Akiva taken from Ben-Akiva et al. (2002) p. 166. 
Bagozzi's action theory model of consumption (2000)

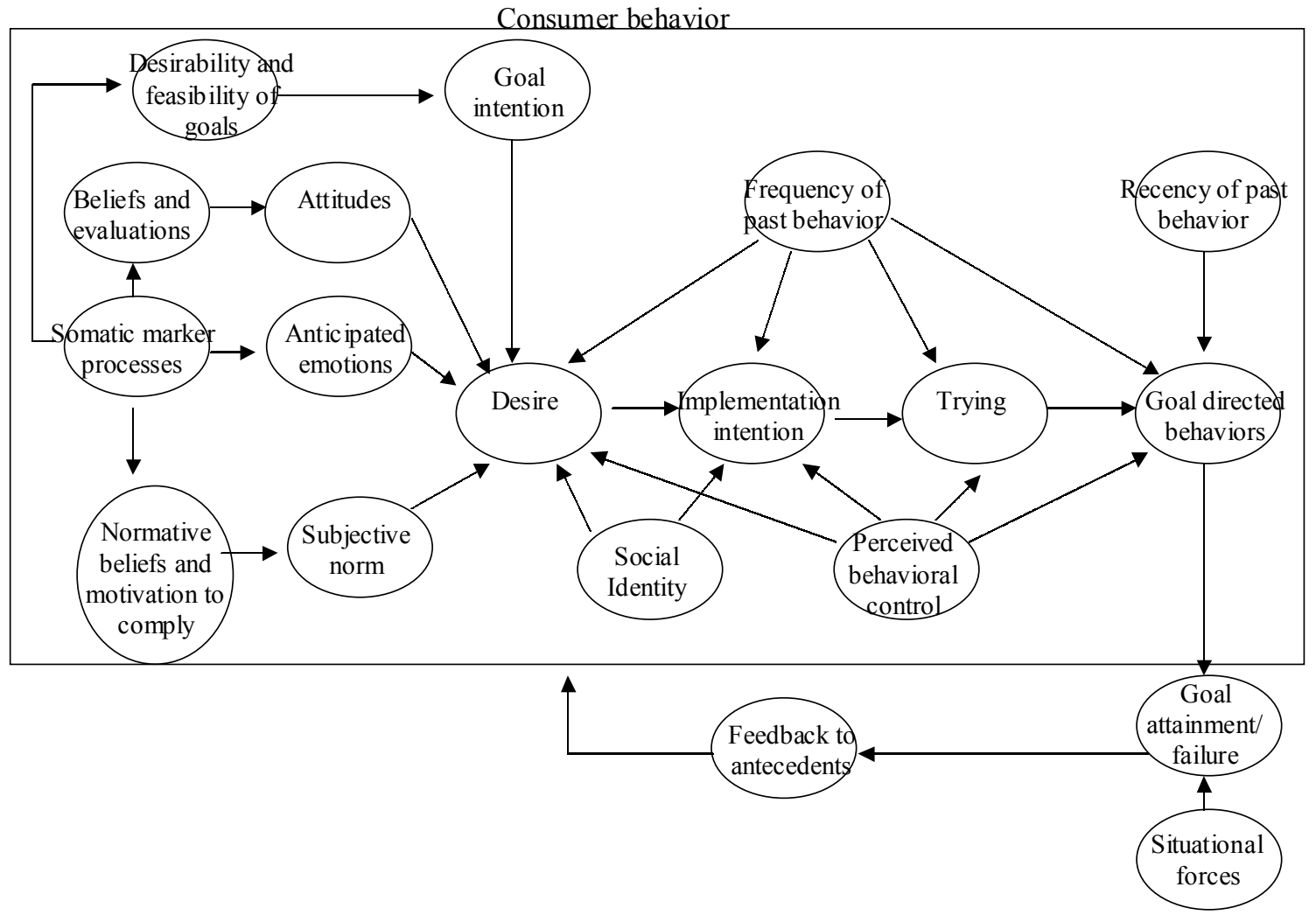

Figure 2.5 Bagozzi's action theory model of consumption taken from Bagozzi (2000) p. 105. 


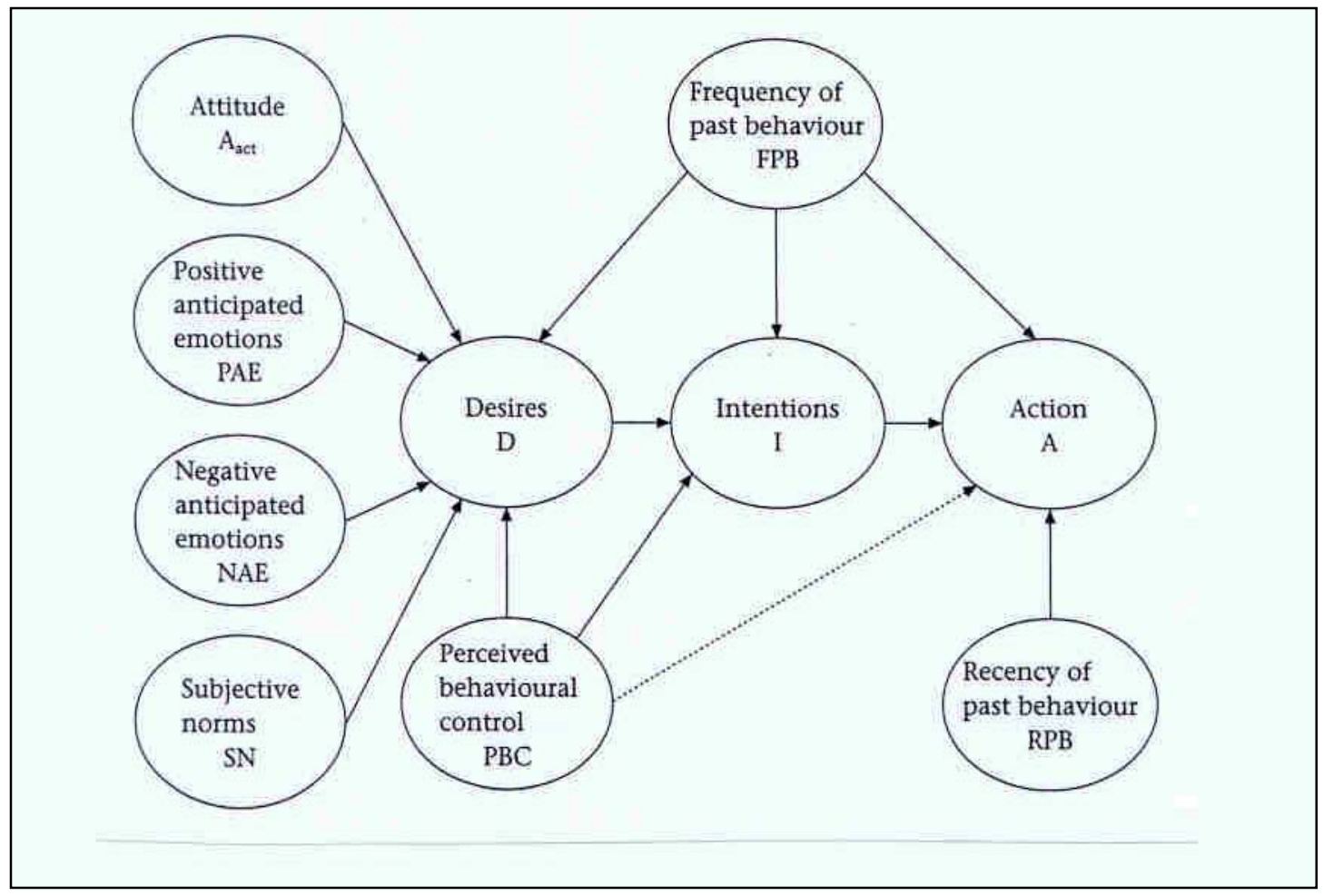

Figure 2.6 Bagozzi's model of goal directed behavior taken from Bagozzi, Gurhan-Canli, and Priester (2002) p. 95. 


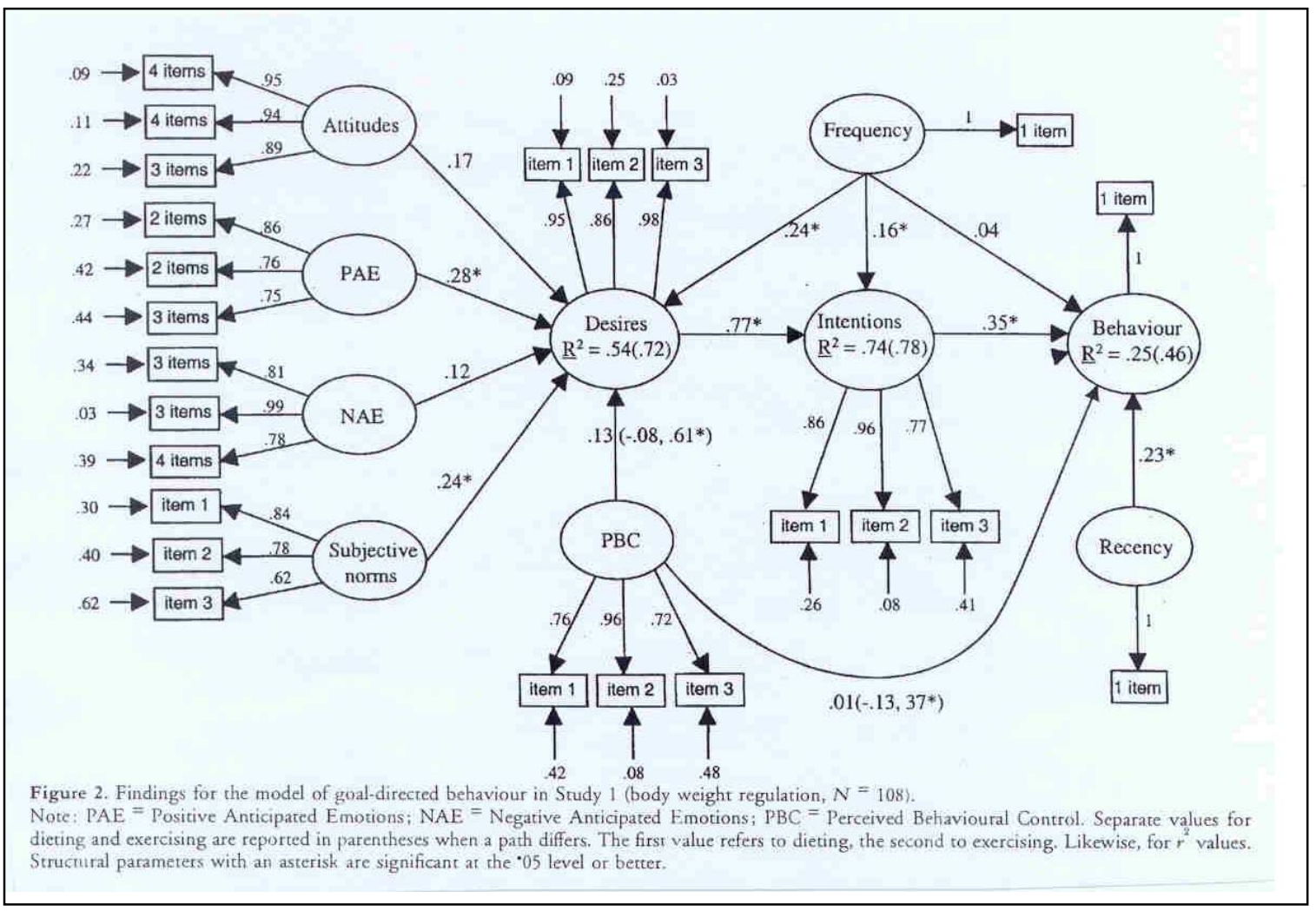

Figure 2.7 Typical LISREL approach of Bagozzi taken from Perugini and Bagozzi (2000), p90. 


\section{CHAPTER 3}

\section{THE MODELS}

This chapter introduces models for individual level variable selection. The first variant, the heterogeneous variable selection model extends the previous literature on Bayesian variable selection to identify at the individual level the most promising subset of variables. The pooled variable selection model uses data from separate contexts and assumes only a subset of variables are used by each individual in one of the contexts. Examples of separate contexts include partial and full profile choice experiments or choice experiments and actual market place transactions. A hybrid model is introduced that incorporates contextual variation into the heterogeneous variable selection model. Finally, the threshold variable selection model incorporates explanatory variables and a specific behavioral structure to determine which variables are selected. Figure 3.1 lists the basic models, their relationship with each other, and the previous literature.

The variable selection models are derived and applied to discrete choice and rank ordered choice data frequently collected in marketing research studies. As is standard, choices or the rank ordering of alternatives is assumed to arise from the latent underlying utility for each alternative. The utility for a choice is assumed to be a linear compensatory function of the product's attributes, and the importance that each individual 
assigns to the attributes. Following random utility theory, this can be represented as $\mathrm{z}_{\mathrm{h}}=$ $\mathrm{X}_{\mathrm{h}} \beta_{\mathrm{h}}+\varepsilon_{\mathrm{h}}$ where $\mathrm{z}_{\mathrm{h}}$ is a vector representing the latent utility for the alternatives, $\beta_{\mathrm{h}}$ is the vector of attribute weights for individual $\mathrm{h}, \mathrm{X}_{\mathrm{h}}$ is the design matrix with the levels of the various product attributes and $\varepsilon_{\mathrm{h}}$ is a stochastic component that is not observed by the analyst. Variable selection seeks to identify which columns of the matrix $X_{h}$ to exclude from the model, or alternatively which elements of $\beta_{\mathrm{h}}$ should be set $=0$. Throughout this document, the terms "variables" and "product attributes" are used interchangeably.

The chapter is organized as follows. Each of the models is presented in turn including the model derivation, estimation algorithms, and simulation results. Markov chain Monte Carlo methods are used for estimating the models and include Gibbs samplers (Casella and George, 1992), the "griddy" Gibbs (Tanner,1993), and random walk and independence chain Metropolis-Hastings algorithms (Chib and Greenberg, 1995). For a comprehensive overview of the use of Bayesian methods in marketing see Rossi and Allenby (2003).

\subsection{Heterogeneous Variable Selection Model}

The heterogeneous variable selection model is an extension of the Bayesian variable selection procedure suggested by George and McCulloch $(1993,1997)$. In marketing datasets it is common to have multiple observations per person in the form of either purchasing history (scanner panel datasets or transaction records) or repeated choice experiments (conjoint analysis). However, too few observations exist to obtain reliable fixed effect models estimated for each individual. Academics in marketing have turned to hierarchical Bayesian models to coherently capture heterogeneity and provide 
inference for both individual level and hyper-parameters (see Allenby and Rossi 1999, Rossi and Allenby, 2003). The current model is formulated to identify at the individual level the most promising subset of variables. This extends the aggregate level variable selection models discussed in the statistics literature.

\subsubsection{Model Derivation}

Bayesian hierarchical modeling has been used in marketing to model heterogeneity. These models assume that each individual's parameters are drawn from a common distribution, the distribution of heterogeneity; the hierarchical model is completed by placing priors on the parameters in the distribution of heterogeneity. The posterior distribution of individual level parameters is proportional to the individual level likelihood and the prior. This is represented as:

$$
\pi\left(\beta_{\mathrm{h}} \mid \mathrm{y}_{\mathrm{h}}\right) \propto l\left(\mathrm{y}_{\mathrm{h}} \mid \beta_{\mathrm{h}}\right) \times \pi\left(\beta_{\mathrm{h}} \mid \bar{\beta}, \mathrm{V}_{\beta}\right) \times \pi(\bar{\beta}) \times \pi\left(\mathrm{V}_{\beta}\right)
$$

where $\bar{\beta}$ and $\mathrm{V}_{\beta}$ are parameters in the distribution of heterogeneity and $\pi(\bar{\beta})$ and $\pi\left(\mathrm{V}_{\beta}\right)$ are prior distributions placed on those parameters. When information in the likelihood is diffuse at the individual level, the posterior will be shrunk to the distribution of heterogeneity. There are instances when it is desirable not to shrink the individual level parameter back to the distribution of heterogeneity. For instance, if utility is modeled as a linear compensatory function of product attributes and importance weights $\left(\beta_{\mathrm{h}} \mathrm{s}\right)$, it may be more informative (and predicatively accurate) to set certain importance weights to 0 than to shrink them towards an overall grand mean.

The heterogeneous variable selection model has a distribution of heterogeneity with mass concentrated at 0 and away from 0 for each parameter. An example of this 
type of distribution of heterogeneity is illustrated in figure 3.2. These graphs depict the value of $\beta_{\mathrm{h}}$ across a sample of 500 respondents. Standard Bayesian variable selection techniques depict the posterior distribution of $\beta$ (an aggregate level parameter) in a similar fashion but the interpretation is very different. In aggregate level analysis the spike at 0 depicts the relative evidence that $\beta$ is "practically" indistinguishable from 0. For the heterogeneous variable selection model, the spike at 0 represents the proportion of the sample for which $\beta_{\mathrm{h}}$ is "practically" indistinguishable from 0 . For marketing managers, knowing who uses which product attributes offers greater opportunity for customizing products and marketing efforts.

A formal representation of the model is derived for multinomial discrete choice data. Let $\mathrm{y}_{\text {hik }}=1$ if person $\mathrm{h}$ selects brand $\mathrm{i}$ on choice occasion $\mathrm{k}$. If $\mathrm{z}_{\mathrm{hik}}$ represents the latent underlying level of utility for $\mathrm{y}_{\mathrm{hik}}$, then $\mathrm{y}_{\mathrm{hik}}=1$ if $\mathrm{z}_{\mathrm{hik}}>\mathrm{z}_{\mathrm{hnk}}$ for all $\mathrm{N}$ alternatives in the choice set. As is common in marketing studies, latent utility is represented as:

$$
\mathrm{Z}_{\mathrm{hik}}=\sum_{j=1}^{J} \beta_{\mathrm{hj}} \mathrm{X}_{\mathrm{hijk}}+\varepsilon_{\mathrm{hik}}
$$

where $x_{\text {hijk }}$ represents the value of variable (or product attribute) $\mathrm{j}$ for brand $\mathrm{i}$, facing person $\mathrm{h}$ on choice occasion $\mathrm{k}$. If $\varepsilon \sim \operatorname{EV}(0,1)$ then the standard multinomial logit choice probability results:

$$
\operatorname{Pr}\left(\mathrm{z}_{\mathrm{hik}}>\mathrm{z}_{\mathrm{hnk}} \text { for all } \mathrm{N}\right)=\frac{\exp \left(\sum_{\mathrm{j}=1}^{\mathrm{J}} \beta_{\mathrm{hj}} \mathrm{x}_{\mathrm{hijk}}\right)}{\sum_{n=1}^{N} \exp \left(\sum_{\mathrm{j}=1}^{\mathrm{J}} \beta_{\mathrm{hj}} \mathrm{x}_{\mathrm{hnjk}}\right)}
$$


Let $l_{\mathrm{h}}$ represent the likelihood for individual $\mathrm{h}$ by taking the product of (13) across the $\mathrm{k}$ choice sets.

Variable selection is handled through the distribution of heterogeneity for $\beta_{\mathrm{h}}$ :

$$
\beta_{\mathrm{h}} \sim \operatorname{MVN}\left(\mathrm{C}_{\tau \mathrm{h}} \bar{\beta}, \mathrm{C}_{\tau \mathrm{h}} \mathrm{V}_{\beta} \mathrm{C}_{\tau \mathrm{h}}\right)
$$

where $\tau_{\mathrm{h}}$ is a vector of length $\mathrm{J}$ and $\tau_{\mathrm{hj}} \in\{\mathrm{c}, 1\}$ where $\mathrm{c}$ is some small constant set by the researcher. $\mathrm{C}_{\tau \mathrm{h}}$ is a $\mathrm{J} \times \mathrm{J}$ matrix formed as $\operatorname{diag}\left[\tau_{\mathrm{h}}\right]$. Following George and McCulloch (1993), when $\tau_{\mathrm{hj}}=\mathrm{c}, \beta_{\mathrm{hj}}$ is drawn from a distribution with the mean and variance for item $\mathrm{j}$ set very close to 0 (depending on the size of c). In simulations and practical applications, setting $\mathrm{c}=0.01$ has worked satisfactorily.

The model hierarchy is completed by specifying priors for the remaining individual parameters and the hyper-parameters.

$$
\begin{aligned}
& \tau_{\mathrm{hj}}=1 \text { with probability } \theta_{\mathrm{j}}, \tau_{\mathrm{hj}}=\mathrm{c} \text { with probability } 1-\theta_{\mathrm{j}} \\
& \bar{\beta} \sim \operatorname{MVN}(0,100 \mathrm{I}) \\
& \mathrm{V}_{\beta} \sim \operatorname{IW}(v, \Delta) \\
& \theta_{\mathrm{j}} \sim \operatorname{Beta}(\mathrm{a}, \mathrm{b})
\end{aligned}
$$

where IW is the inverted Wishart distribution with $v=J+8$, and $\Delta=v I$ and $a=b=1$. Consistent with previous work in this area, the probability that any variable is selected is independent of the other variables in the model.

\subsubsection{Estimation Algorithm}

Estimating the model specified in $(13)$ - (18) requires special attention is paid to drawing the individual level $\tau_{\mathrm{h}}$ and $\beta_{\mathrm{h}}$ as well as the hyper-parameters $\bar{\beta}$ and $\mathrm{V}_{\beta}$. 
Standard Metropolis-Hastings algorithms for the non-conjugate multinomial logit model draw $\beta_{\mathrm{h}}$ conditional on all the other model parameters using a random walk algorithm. See for instance Arora, Allenby, and Ginter (1998). However, if $\tau_{\mathrm{hj}}=\mathrm{c}$ the prior distribution will place a great deal of mass near the neighborhood $\beta_{\mathrm{hj}}=0$. Similarly, drawing $\tau_{\mathrm{hj}} \mid \beta_{\mathrm{hj}}$ when the current draw $\left|\beta_{\mathrm{hj}}\right| \gg>0$ may unduly penalize moves to $\tau_{\mathrm{hj}}=\mathrm{c}$. As a practical matter, once the chain enters the state $\tau_{\mathrm{hj}}=\mathrm{c}$, it may become "stuck" and navigate the posterior space very slowly. At the extreme, if $\mathrm{c}=0$ the chain becomes irreducible as noted by George and McCulloch (1997).

In certain cases it is possible to analytically or numerically marginalize the posterior distribution for $\tau_{\mathrm{h}}$. Conceptually, the posterior marginal distribution is obtained as:

$$
\pi\left(\tau_{\mathrm{h}} \mid \text { Data }\right)=\int \operatorname{pr}\left(\tau_{\mathrm{h}}, \Theta \mid \text { Data }\right) \operatorname{pr}(\Theta) \mathrm{d} \Theta
$$

where $\Theta$ represents all other model parameters. George and McCulloch (1997), Geweke (1996), and Raftery et al. (1997) did this in the case of an aggregate linear regression model with conjugate priors. As demonstrated by Geweke (1996) and Raftery et al. (1997) when the posterior is marginalized, the restriction on $\mathrm{c} \neq 0$ can be lifted and the prior can be modeled as a mixture of a degenerate distribution with point mass at 0 and a continuous normal distribution. See Hoeting et al. (1999) for more details and other examples applied to aggregate level models. In models with heterogeneity, $\Theta$ includes $\beta_{\mathrm{h}}, \bar{\beta}, \mathrm{V}_{\beta}, \theta$, as well as the priors on $\bar{\beta}, \mathrm{V}_{\beta}$, and $\theta$ making the evaluation of (19) seemingly intractable. 
To ensure proper mixing of the MCMC chain and to avoid evaluation of the marginal posterior of $\tau_{\mathrm{h}}$, an independence chain Metropolis-Hastings (M-H) algorithm is proposed. Similar in spirit to Geweke (1996), the algorithm draws $\beta_{\mathrm{h}}$ and $\tau_{\mathrm{h}}$ together. The candidate generating distribution is the prior $\pi\left(\beta_{\mathrm{h}}, \tau_{\mathrm{h}} \mid \bar{\beta}, \mathrm{V}_{\beta}, \theta\right)$ formed as $\pi\left(\beta_{\mathrm{h}} \mid \tau_{\mathrm{h}}\right.$, $\left.\bar{\beta}, V_{\beta}\right) \times \pi\left(\tau_{h} \mid \theta\right)$ and moves to new values of $\beta_{\mathrm{h}}$ and $\tau_{\mathrm{h}}$ are evaluated jointly in the acceptance probability of the M-H algorithm. Details are given below.

The second issue addressed by the algorithm is drawing $\bar{\beta}$ and $V_{\beta}$. Each $\beta_{\mathrm{h}}$ has a unique prior depending on $\tau_{\mathrm{h}}$, e.g. $\beta_{\mathrm{h}} \sim \operatorname{MVN}\left(\mathrm{C}_{\tau \mathrm{h}} \bar{\beta}, \mathrm{C}_{\tau \mathrm{h}} \mathrm{V}_{\beta} \mathrm{C}_{\tau \mathrm{h}}\right)$. Therefore standard methods are no longer applicable. However, we can condition on both $\left\{\tau_{\mathrm{h}}\right\}$ and $\left\{\beta_{\mathrm{h}}\right\}$ when drawing $\bar{\beta}$ and $\mathrm{V}_{\beta}$. Let $\beta_{h}^{*}=C_{\tau h}^{-1} \beta_{\mathrm{h}}$ and note that $\beta_{h}^{*} \sim \operatorname{MVN}\left(C_{\tau h}^{-1} \mathrm{C}_{\tau \mathrm{h}} \bar{\beta}\right.$, $\left.C_{\tau h}^{-1} \mathrm{C}_{\tau \mathrm{h}} \mathrm{V}_{\beta} C_{\tau h}^{-1} \mathrm{C}_{\tau \mathrm{h}}\right)$ which is equivalent to $\beta_{h}^{*} \sim \operatorname{MVN}\left(\bar{\beta}, \mathrm{V}_{\beta}\right)$. Standard methods can now be used on the transformed vectors $\left\{\beta_{h}^{*}\right\}$.

The following steps describe an MCMC chain with the posterior distribution of all model parameters as the stationary distribution.

1. Generate $\beta_{\mathrm{h},} \tau_{\mathrm{h}} \mid \bar{\beta}, \mathrm{V}_{\beta}, \theta, \mathrm{y}_{\mathrm{h}}, \mathrm{X}_{\mathrm{h}}$ for $\mathrm{h}=1, \ldots, \mathrm{H}$

An independence chain M-H step is used. Let $\tau_{h}^{(n)}$ represent a new candidate vector and $\tau_{h}^{(o)}$ represent the old vector from the previous iteration of the chain. Similar notation is used for $\beta_{h}^{(n)}$ and $\beta_{h}^{(o)}$. 
a) Set $\tau_{h j}^{(n)}=1$ with probability $\theta_{\mathrm{j}}$, otherwise $\tau_{h j}^{(n)}=\mathrm{c}$ for $\mathrm{j}=1, \ldots, \mathrm{J}$

b) Draw $\beta_{h}^{(n)}$ from $\operatorname{MVN}\left(C_{\tau h}^{(n)} \bar{\beta}, C_{\tau h}^{(n)} V_{\beta} C_{\tau h}^{(n)}\right)$

Accept the new values $\tau_{h}^{(n)}$ and $\beta_{h}^{(n)}$ with probability:

$$
\operatorname{Pr}(\text { accept })=\min \left(\frac{l_{h}\left(\beta_{h}^{(n)}, \tau_{h}^{(n)}\right)}{l_{h}\left(\beta_{h}^{(o)}, \tau_{h}^{(o)}\right)}, 1\right)
$$

Note that since the prior, in this case the distribution heterogeneity, is used to generate the candidate, it is not used to evaluate the acceptance probability.

2. Generate $\bar{\beta} \mid\left\{\tau_{\mathrm{h}}\right\},\left\{\beta_{\mathrm{h}}\right\}, \mathrm{V}_{\beta}$

Form $\beta_{h}^{*}$ by $C_{\tau h}^{-1} \beta_{\mathrm{h}}$.

$$
\begin{aligned}
& \bar{\beta} \sim \operatorname{MVN}\left(\overline{\mathrm{b}},\left(\left(\mathrm{V}_{\beta} / \mathrm{H}\right)^{-1}+(100 \mathrm{I})^{-1}\right)^{-1}\right) \\
& \left.\overline{\mathrm{b}}=\left(\left(\mathrm{V}_{\beta} / \mathrm{H}\right)^{-1}+(100 \mathrm{I})^{-1}\right)^{-1}\right)\left(\mathrm{V}_{\beta}^{-1} \sum_{h=1}^{H} \beta_{h}^{*}+(100 \mathrm{I})^{-1}(0)\right)
\end{aligned}
$$

3. Generate $\mathrm{V}_{\beta} \mid\left\{\tau_{\mathrm{h}}\right\},\left\{\beta_{\mathrm{h}}\right\}, \bar{\beta}$

Form $\beta_{h}^{*}$ by $C_{\tau h}^{-1} \beta_{\mathrm{h}}$.

$$
\mathrm{V}_{\beta} \sim \operatorname{IW}\left(v+\mathrm{H}, \Delta+\sum_{h=1}^{H}\left(\beta_{h}^{*}-\bar{\beta}\right)^{\prime}\left(\beta_{h}^{*}-\bar{\beta}\right)\right)
$$


4. Generate $\theta_{\mathrm{j}} \mid\left\{\tau_{\mathrm{hj}}\right\}$ for $\mathrm{j}=1, \ldots \mathrm{J}$

Let $\mathrm{s}_{\mathrm{hj}}=1$ if $\tau_{\mathrm{hj}}=1,0$ otherwise.

$$
\theta_{\mathrm{j}} \sim \operatorname{Binomial}\left(\mathrm{a}+\sum_{\mathrm{h}=1}^{\mathrm{H}} \mathrm{s}_{\mathrm{hj}}, \mathrm{H}-\sum_{\mathrm{h}=1}^{\mathrm{H}} \mathrm{s}_{\mathrm{hj}}+\mathrm{b}\right)
$$

\subsubsection{Simulation Results}

This section reports on the results of a simulation study that demonstrates that the model is identified and the parameters can be recovered. Data was simulated for 500 individuals facing 20 multinomial choice tasks with four alternatives per task. Similar results were obtained with as few as 5 choice tasks per individual. Explanatory variables for each alternative, $\mathbf{x}_{\mathrm{hijk}}$, were generated from a uniform distribution. For identification, the vector of $\mathrm{x}_{\text {hik }}$ is set equal to 0 for the fourth alternative in each choice set $\mathrm{k}$.

Individual parameters $\tau_{\mathrm{h}}$ and then $\beta_{\mathrm{h}}$ are drawn from the appropriate distributions and choices are simulated according to the model specified above.

The MCMC chain described in section 3.1.2 was run on the simulated data and

results are presented in table 3.1. All hyper-parameters, $\bar{\beta}, \mathrm{V}_{\beta}$, and $\theta$ are recovered within $+/-2$ posterior standard deviations of the actual values. It is instructive to look at the results from a model that ignores the variable selection problem. In the bottom half of table 3.1 are the results from a standard model with the assumption $\beta_{\mathrm{h}} \sim \operatorname{MVN}\left(\bar{\beta}, \mathrm{V}_{\beta}\right)$. Ignoring variable selection results in posterior means for $\bar{\beta}$ shrunk towards 0 and an inflated distribution of unexplained heterogeneity $\mathrm{V}_{\beta}$. While the multivariate normal 
prior is sufficiently flexible to capture various forms of heterogeneity, a mis-specified prior can lead to very different posterior inferences.

The heterogeneous variable selection model is proposed to handle distributions of heterogeneity with mass at 0 and away from 0 for different parameters across respondents. Identifying which parameters are practically indistinguishable from 0 for certain consumers offers additional managerial insights for either new product development or product positioning. Incorporating heterogeneity into the model presents challenges that are not present in the aggregate variable selection models presented in the statistics literature. A tractable algorithm for estimating the model has been presented that overcomes these difficulties.

\subsection{Pooled Variable Selection Model}

This section presents a model for variable selection that incorporates changes in the response context. Many studies in marketing collect information across several different contexts. For instance, experimental choice studies may be followed-up with longitudinal or self-reported instances of actual brand choice. Partial profile conjoint exercises may be followed by full profile choice sets in the same experimental study. As the response context changes, respondents may use different sets of variables.

Similar to the heterogeneous variable selection model, the pooled variable selection model provides individual level estimates of attribute weights $\left(\beta_{\mathrm{h}}\right.$ 's) and allows the product attributes used to be individual specific. In the heterogeneous variable selection model, a respondent uses a subset of product attributes for all choice/ranking tasks. By comparison, in the pooled variable selection model, respondents are assumed 
to use all attributes in one choice context, but only a subset in the targeted context. The data is pooled across contexts and the model identifies which variables are used in the targeted setting.

\subsubsection{Model Derivation}

The derivation of the pooled variable selection model assumes that in an experimental setting such as a partial profile conjoint exercise, participants give "good" answers to survey questions. Thus the product attribute weights, the $\beta_{\mathrm{h}}$ 's, are a meaningful quantity on which to base inference and predictions. However, when the context changes from either an experimental setting and/or a setting where the consumer is faced with the full array of product attributes, he or she selects only a subset of attributes to use in a brand choice decision.

The model is derived assuming two sets of data are available to the researcher. In the first set of data it is assumed that the respondent is using all the variables presented either because it is a partial profile conjoint exercise or some other experimental manipulation ensures this outcome. In the second set of data, it is assumed that the respondent may be using a subset of variables or product attributes. The following assumes that a rank-ordered conjoint task is used for the first set of data and one or more full profile multinomial choice outcomes are available for the second set of data.

The model is represented as follows. Latent utility for the rank ordered conjoint task is represented as:

$$
\mathrm{U}_{\text {him }}=\sum_{j=1}^{J} \beta_{\mathrm{hj}} \mathrm{d}_{\text {hijm }}+\xi_{\text {him }}
$$


where $\mathrm{h}$ indexes the individual or household, $\mathrm{m}$ indexes the choice task, $\mathrm{i}$ indexes the alternative in choice task $\mathrm{m}$, and $\mathrm{j}$ indexes the product attributes. $\mathrm{d}_{\mathrm{hijm}}$ represents the level of the $\mathrm{j}^{\text {th }}$ product attribute for alternative $\mathrm{i}$, presented to household $\mathrm{h}$, in choice set $\mathrm{m}$. Latent utility for the full profile brand choice decision is represented as:

$$
\mathrm{Z}_{\mathrm{hik}}=\sum_{j=1}^{J} \beta_{\mathrm{hj}} \lambda_{\mathrm{hj}} \mathrm{X}_{\mathrm{hijk}}+\varepsilon_{\mathrm{hik}}
$$

where the notation follows from above but with $\mathrm{k}$ choice occasions available for study. Here $\lambda_{\mathrm{hj}}$ is a scalar equal to 1 if household $\mathrm{h}$ uses attribute $\mathrm{j}$ in the full profile choice task, otherwise it equals 0 .

Assuming the $\xi$ and $\varepsilon$ are identically and independently distributed $\mathrm{EV}(0,1)$ leads to ordered logit and multinomial logit likelihoods for the observed rank orderings and brand choices, respectively. Following Chapman and Staelin (1982) the ordered logit likelihood for individual $\mathrm{h}$ for choice set $\mathrm{m}$ is represented as:

$$
\operatorname{Pr}\left(\mathrm{V}_{\mathrm{h} 1 \mathrm{~m}}>\mathrm{V}_{\mathrm{h} 2 \mathrm{~m}}>\ldots>\mathrm{V}_{\mathrm{hIm}}\right)=\prod_{i=1}^{I-1} \frac{\exp \left(\mathrm{d}_{\mathrm{him}}{ }^{\prime} \beta_{h}\right)}{\sum_{n=i}^{I} \exp \left(\mathrm{d}_{\mathrm{hnm}}{ }^{\prime} \beta_{h}\right)}
$$

where $V_{\text {him }}$ represents the rank order of alternatives. The multinomial logit choice probability for $\mathrm{y}_{\text {hik }}=1$ is represented as follows:

$$
\operatorname{Pr}\left(\mathrm{Z}_{\mathrm{hik}}>\mathrm{Z}_{\mathrm{hnk}} \text { for all } \mathrm{N}\right)=\frac{\exp \left(\sum_{\mathrm{j}=1}^{\mathrm{J}} \beta_{\mathrm{hj}} \lambda_{h j} \mathrm{x}_{\mathrm{hijk}}\right)}{\sum_{n=1}^{N} \exp \left(\sum_{\mathrm{j}=1}^{\mathrm{J}} \beta_{\mathrm{hj}} \lambda_{h j} \mathrm{x}_{\mathrm{hnjk}}\right)}
$$

Let $l_{l h}$ represent the product of equation (9) across the m ordered choice sets for individual $\mathrm{h}$ and $l_{2 h}$ represent the product of equation (10) across the k multinomial 
choice sets for individual $\mathrm{h}$. The likelihood for an individual is then represented by $l_{h}=l_{l h}$ $\times l_{2 h}$.

Heterogeneity for individual level model parameters is specified as follows:

$$
\begin{aligned}
& \beta_{\mathrm{h}} \sim \operatorname{MVN}\left(\bar{\beta}, \mathrm{V}_{\beta}\right) \\
& \lambda_{\mathrm{hj}} \sim \operatorname{Bernoulli}\left(\theta_{\mathrm{j}}\right)
\end{aligned}
$$

The hierarchical model is completed by choosing priors for the hyper-parameters:

$$
\begin{aligned}
& \bar{\beta} \sim \operatorname{MVN}(0,100 \mathrm{I}) \\
& \mathrm{V}_{\beta} \sim \operatorname{IW}(v, \Delta) \\
& \theta_{\mathrm{j}} \sim \operatorname{Beta}(\mathrm{a}, \mathrm{b})
\end{aligned}
$$

where IW is the inverted Wishart distribution with $v=\mathrm{J}+8$, and $\Delta=v \mathrm{I}$ and $\mathrm{a}=\mathrm{b}=1$. As in the heterogeneous variable selection model, the probability that any variable is selected is independent of the other variables in the model.

\subsubsection{Estimation Algorithm}

Estimation of the pooled variable selection model in equations (22)-(28) is straightforward and relies on standard techniques.

1. Generate $\beta_{\mathrm{h}} \mid \lambda_{\mathrm{h}}, \bar{\beta}, \mathrm{V}_{\beta}, \mathrm{y}_{\mathrm{h}}, \mathrm{X}_{\mathrm{h}}, \mathrm{D}_{\mathrm{h}}, \mathrm{V}_{\mathrm{h}}$ for $\mathrm{h}=1, \ldots, \mathrm{H}$

A random walk Metropolis-Hastings algorithm is used to draw from the posterior

distribution. A candidate vector $\beta_{h}^{(n)}$ is formed as $\beta_{h}^{(n)}=\beta_{h}^{(o)}+\zeta$ where $\zeta \sim \operatorname{MVN}(0, \mathrm{wI})$ and $\mathrm{w}$ is chosen to ensure a 50\% acceptance rate. $\beta_{h}^{(o)}$ represents the previous, or "old" draw of $\beta_{\mathrm{h}}$. The new parameter is accepted with probability: 


$$
\operatorname{Pr}(\text { accept })=\min \left(\frac{l_{h}\left(\beta_{h}^{(n)}\right) \times \exp \left(-\frac{1}{2}\left(\beta_{h}^{(n)}-\bar{\beta}\right)^{\prime} \mathrm{V}_{\beta}^{-1}\left(\beta_{h}^{(n)}-\bar{\beta}\right)\right)}{l_{h}\left(\beta_{h}^{(o)}\right) \times \exp \left(-\frac{1}{2}\left(\beta_{h}^{(o)}-\bar{\beta}\right)^{\prime} \mathrm{V}_{\beta}^{-1}\left(\beta_{h}^{(o)}-\bar{\beta}\right)\right)}, 1\right)
$$

2. Generate $\lambda_{\mathrm{hj}} \mid \lambda_{\mathrm{h}-\mathrm{j}}, \theta_{\mathrm{j}}, \beta_{\mathrm{h}}, \mathrm{y}_{\mathrm{h}}, \mathrm{X}_{\mathrm{h}}$ for $\mathrm{j}=1, \ldots, \mathrm{J}$ and $\mathrm{h}=1, \ldots \mathrm{H}$

Here $\lambda_{\mathrm{h}-\mathrm{j}}$ represents all other elements of $\lambda_{\mathrm{h}}$ other than $\mathrm{j}$. Either a griddy Gibbs or independence chain M-H may be used. Here the griddy Gibbs is useful since the support for $\lambda_{\mathrm{hj}}$ takes on only two values, $(0,1)$. Let $\lambda_{h j}^{(1)}$ represent $\lambda_{\mathrm{hj}}=1$ and $\lambda_{h j}^{(0)}$ represent $\lambda_{\mathrm{hj}}=0$.

$$
\operatorname{Pr}\left(\lambda_{\mathrm{hj}}=1\right)=\left(\frac{l_{h 2}\left(\lambda_{h j}^{(1)}\right) \times \theta_{j}}{\left(l_{h 2}\left(\lambda_{h j}^{(1)}\right) \times \theta_{j}\right)+\left(l_{h 2}\left(\lambda_{h j}^{(0)}\right) \times\left(1-\theta_{j}\right)\right)}\right)
$$

For an independence chain $\mathrm{M}-\mathrm{H}$, draw $\lambda_{h j}^{(n)}$ from the Bernoulli $\left(\theta_{\mathrm{j}}\right)$ and accept the new

draw with probability:

$$
\operatorname{Pr}(\text { accept })=\min \left(\frac{l_{h 2}\left(\lambda_{h j}^{(n)}\right)}{l_{h 2}\left(\lambda_{h j}^{(o)}\right)}, 1\right)
$$

In both simulations and practical applications either method has performed satisfactorily. Note that only the likelihood from the second part of the data, the full profile task, is used in drawing $\lambda_{\mathrm{h}}$. 
3. Generate $\bar{\beta} \mid\left\{\beta_{\mathrm{h}}\right\}, \mathrm{V}_{\beta}$

$$
\begin{aligned}
& \bar{\beta} \sim \operatorname{MVN}\left(\overline{\mathrm{b}},\left(\left(\mathrm{V}_{\beta} / \mathrm{H}\right)^{-1}+(100 \mathrm{I})^{-1}\right)^{-1}\right) \\
& \left.\overline{\mathrm{b}}=\left(\left(\mathrm{V}_{\beta} / \mathrm{H}\right)^{-1}+(100 \mathrm{I})^{-1}\right)^{-1}\right)\left(\mathrm{V}_{\beta}^{-1} \sum_{h=1}^{H} \beta_{h}+(100 \mathrm{I})^{-1}(0)\right)
\end{aligned}
$$

4. Generate $\mathrm{V}_{\beta} \mid\left\{\beta_{\mathrm{h}}\right\}, \bar{\beta}$

$$
\mathrm{V}_{\beta} \sim \operatorname{IW}\left(\nu+\mathrm{H}, \Delta+\sum_{h=1}^{H}\left(\beta_{h}-\bar{\beta}\right)^{\prime}\left(\beta_{h}-\bar{\beta}\right)\right)
$$

5. Generate $\theta_{\mathrm{j}} \mid\left\{\lambda_{\mathrm{hj}}\right\}$ for $\mathrm{j}=1, \ldots \mathrm{J}$

Let $\mathrm{s}_{\mathrm{hj}}=1$ if $\tau_{\mathrm{hj}}=1,0$ otherwise.

$$
\theta_{\mathrm{j}} \sim \operatorname{Binomial}\left(\mathrm{a}+\sum_{\mathrm{h}=1}^{\mathrm{H}} \mathrm{s}_{\mathrm{hj}}, \mathrm{H}-\sum_{\mathrm{h}=1}^{\mathrm{H}} \mathrm{s}_{\mathrm{hj}}+\mathrm{b}\right)
$$

\subsubsection{Simulation Results}

This section reports on the results of a simulation study that demonstrates that the pooled variable selection model is identified and the parameters can be recovered. Data was simulated for 500 individuals facing 5 rank ordered choice tasks with four alternatives per task and a single multinomial choice task. Explanatory variables for each alternative, $\mathrm{d}_{\mathrm{hijm}}$ and $\mathrm{x}_{\mathrm{hijk}}$, were generated from a uniform distribution. For identification, the values of the explanatory variables are all set to 0 for the fourth alternative in each choice set. Individual parameters $\lambda_{h}$ and $\beta_{h}$ are drawn from the appropriate distribution and rank orderings and choices are simulated according to the model specified above. 
The MCMC chain described above with the independence chain M-H algorithm for drawing $\lambda_{\mathrm{h}}$ was used on the simulated data. Results are presented in table 3.2. Even with only one multinomial brand choice, all parameter values are recovered within $+/-2$ posterior standard deviations of the actual values.

The pooled variable selection model is appropriate when two sets of choice data are available and a "regime" shift at the individual level is envisioned. This "regime" shift may result from moving from a partial profile to a full profile choice task or moving from the artificiality of an experimental choice task to an actual marketplace transaction. In either case the pooled variable selection model identifies which product attributes are selected and used by individuals in the targeted choice task, typically the full profile or actual choice setting. In the next section, a hybrid model combining the heterogeneous variable selection model and the pooled variable selection model is discussed.

\subsection{Hybrid Model}

The hybrid model combines aspects of both the heterogeneous and pooled variable selection models. Specifically, the hybrid model assumes two data sets are available for analysis and a "regime" shift at the individual level occurs as respondents move from one task to the other. Unlike the pooled model, the hybrid model does not assume that respondents use all of the product attributes in the first data set. So, variable selection occurs for both sets of data, consistent with the heterogeneous variable selection model. However, in the targeted task, respondents may use the same subset of variables or a smaller subset of variables. 
For example, consider a market research study that has both partial and full profile conjoint tasks involving a total of 15 product attributes. Assume an appropriate partial profile experimental design is used such that each choice set consists of products described on 8 attributes. Analyzing just the partial profile tasks provides estimates of all 15 product weights. The pooled model assumes individuals used all 15 product attributes in the partial profile choice tasks, but use only a subset of those in the full profile task. The hybrid model relaxes this first assumption and allows respondents to select only a subset of the 15 product attributes to use in the partial profile tasks. The hybrid model then assumes individuals will use the same subset or a smaller subset in the full profile task. So an individual may use only 5 product attributes to make choices in the partial profile tasks and may use the same 5 , or a subset of those 5 attributes when making choices from the full profile task.

\subsubsection{Model Derivation}

The hybrid model is derived assuming that both sets of data involve multinomial choices, although this is not required. Let $y_{\text {him }}^{p}$ represent the observed choices from the first choice exercise and $y_{\text {hik }}^{f}$ represent the observed choices from the second choice exercise. Following established notation, let latent utility be represented as:

$$
\begin{aligned}
& z_{\text {him }}^{p}=\sum_{j=1}^{J} \beta_{\mathrm{hj}} \mathrm{d}_{\mathrm{hijm}}+\xi_{\text {him }} \\
& z_{\text {hik }}^{f}=\sum_{j=1}^{J} \beta_{\mathrm{hj}} \lambda_{\mathrm{hj}} \mathrm{x}_{\mathrm{hijk}}+\varepsilon_{\mathrm{hik}}
\end{aligned}
$$


Assuming the $\xi$ and $\varepsilon$ are identically and independently distributed $\operatorname{EV}(0,1)$ leads to multinomial logit likelihoods for both sets of data.

$$
\begin{aligned}
& \operatorname{Pr}\left(z_{h i m}^{p}>z_{h n m}^{p} \text { for all } \mathrm{N}\right)=\frac{\exp \left(\sum_{\mathrm{j}=1}^{\mathrm{J}} \beta_{\mathrm{hj}} d_{\mathrm{hijm}}\right)}{\sum_{n=1}^{N} \exp \left(\sum_{\mathrm{j}=1}^{\mathrm{J}} \beta_{\mathrm{hj}} d_{\mathrm{hnjm}}\right)} \\
& \operatorname{Pr}\left(z_{h i k}^{f}>z_{h n k}^{f} \text { for all } \mathrm{N}\right)=\frac{\exp \left(\sum_{\mathrm{j}=1}^{\mathrm{J}} \beta_{\mathrm{hj}} \lambda_{h j} \mathrm{x}_{\mathrm{hijk}}\right)}{\sum_{n=1}^{N} \exp \left(\sum_{\mathrm{j}=1}^{\mathrm{J}} \beta_{\mathrm{hj}} \lambda_{h j} \mathrm{x}_{\mathrm{hnjk}}\right)}
\end{aligned}
$$

Let $l_{1 h}$ represent the product of equation (31) across the first m multinomial choice sets for individual $\mathrm{h}$ and $l_{2 h}$ represent the product of equation (32) across the second $\mathrm{k}$ multinomial choice sets for individual $h$. The likelihood for an individual is then represented by $l_{h}=l_{1 h} \times l_{2 h}$.

Similar to the pooled model, $\lambda_{\mathrm{hj}}$ takes care of variable selection for the second part of the data. Consistent with the heterogeneous variable selection model, variable selection across both data sets is handled through the distribution of heterogeneity:

$$
\beta_{\mathrm{h}} \sim \operatorname{MVN}\left(\mathrm{C}_{\tau \mathrm{h}} \bar{\beta}, \mathrm{C}_{\tau \mathrm{h}} \mathrm{V}_{\beta} \mathrm{C}_{\tau \mathrm{h}}\right)
$$

where $\tau_{\mathrm{h}}$ is vector of length $\mathrm{J}$ and $\tau_{\mathrm{hj}} \in\{\mathrm{c}, 1\}$ where $\mathrm{c}$ is some small constant set by the researcher. $\mathrm{C}_{\tau \mathrm{h}}$ is a $\mathrm{J} \times \mathrm{J}$ matrix formed as $\operatorname{diag}\left[\tau_{\mathrm{h}}\right]$. The model hierarchy is completed by specifying priors for the remaining individual parameters and the hyper-parameters. 


$$
\begin{aligned}
& \tau_{\mathrm{hj}}=1 \text { with probability } \theta_{\mathrm{j}}, \tau_{\mathrm{hj}}=\mathrm{c} \text { with probability } 1-\theta_{\mathrm{j}} \\
& \lambda_{\mathrm{hj}}=1 \mid \tau_{\mathrm{hj}}=1 \text { with probability } \phi_{\mathrm{j}} \\
& \lambda_{\mathrm{hj}}=0 \mid \tau_{\mathrm{hj}}=1 \text { with probability } 1-\phi_{\mathrm{j}} \\
& \lambda_{\mathrm{hj}}=1 \mid \tau_{\mathrm{hj}}=0 \text { with probability } 0 \\
& \lambda_{\mathrm{hj}}=0 \mid \tau_{\mathrm{hj}}=0 \text { with probability } 1 \\
& \bar{\beta} \sim \operatorname{MVN}(0,100 \mathrm{I}) \\
& \mathrm{V}_{\beta} \sim \operatorname{IW}(v, \Delta) \\
& \theta_{\mathrm{j}} \sim \operatorname{Beta}(\mathrm{a}, \mathrm{b}) \\
& \phi_{\mathrm{j}} \sim \operatorname{Beta}(\mathrm{a}, \mathrm{b})
\end{aligned}
$$

where IW is the inverted Wishart distribution with $v=\mathrm{J}+8$, and $\Delta=v \mathrm{I}$ and $\mathrm{a}=\mathrm{b}=1$. The probability that any variable is selected is independent of the other variables in the model.

\subsubsection{Estimation Algorithm}

The estimation algorithm for the model (31) - (39) follows the same general scheme as the heterogeneous and pooled variable selection models. Many of the same concerns and techniques apply. 
1. Generate $\beta_{\mathrm{h}}, \tau_{\mathrm{h}} \mid \lambda_{\mathrm{h}}, \bar{\beta}, \mathrm{V}_{\beta}, \theta, \phi, y_{h}^{p}, y_{h}^{f}, \mathrm{X}_{\mathrm{h}}, \mathrm{D}_{\mathrm{h}}$ for $\mathrm{h}=1, \ldots, \mathrm{H}$

An independence chain M-H step is used. Let $\tau_{h}^{(n)}$ represent a new candidate vector and $\tau_{h}^{(o)}$ represent the old vector from the previous iteration of the chain. Similar notation is used for $\beta_{h}^{(n)}$ and $\beta_{h}^{(o)}$.

i) For $\mathrm{j}=1, \ldots, \mathrm{J}$ (See Appendix A for the derivation.)

a. Set $\tau_{h j}^{(n)}=1$ with probability 1 if $\lambda_{\mathrm{hj}}=1$

b. Set $\tau_{h j}^{(n)}=1$ with probability $\frac{\left(1-\phi_{j}\right) \times \theta_{j}}{1-\left(\phi_{j} \times \theta_{j}\right)}$ if $\lambda_{\mathrm{hj}}=0$, otherwise

$$
\tau_{h j}^{(n)}=0
$$

ii) Draw $\beta_{h}^{(n)}$ from $\operatorname{MVN}\left(C_{\tau h}^{(n)} \bar{\beta}, C_{\tau h}^{(n)} V_{\beta} C_{\tau h}^{(n)}\right)$

Accept the new values $\tau_{h}^{(n)}$ and $\beta_{h}^{(n)}$ with probability:

$$
\operatorname{Pr}(\text { accept })=\min \left(\frac{l_{h}\left(\beta_{h}^{(n)}, \tau_{h}^{(n)}\right)}{l_{h}\left(\beta_{h}^{(o)}, \tau_{h}^{(o)}\right)}, 1\right)
$$

Note that since the prior, in this case the distribution heterogeneity, is used to generate the candidate, it is not used to evaluate the acceptance probability.

2. Generate $\lambda_{\mathrm{hj}} \mid \lambda_{\mathrm{h}-\mathrm{j}}, \phi_{\mathrm{j}}, \tau_{\mathrm{hj}}, \beta_{\mathrm{h}}, y_{h}^{f}, \mathrm{X}_{\mathrm{h}}$ for $\mathrm{j}=1, \ldots, \mathrm{J}$ and $\mathrm{h}=1, \ldots \mathrm{H}$

A modified griddy Gibbs sampler is used. Let $\lambda_{h j}^{(1)}$ represent $\lambda_{\mathrm{hj}}=1$ and $\lambda_{h j}^{(0)}$ represent $\lambda_{\mathrm{hj}}$ $=0$. 
a) If $\tau_{\mathrm{hj}}=0$, then $\lambda_{\mathrm{hj}}=0$.

b) If $\tau_{\mathrm{hj}}=0$, then

$$
\operatorname{Pr}\left(\lambda_{\mathrm{hj}}=1\right)=\frac{l_{h 2}\left(\lambda_{h j}^{(1)}\right) \times \phi_{j}}{\left(l_{h 2}\left(\lambda_{h j}^{(1)}\right) \times \phi_{j}\right)+\left(l_{h 2}\left(\lambda_{h j}^{(0)}\right) \times\left(1-\phi_{j}\right)\right)}
$$

3. Generate $\bar{\beta} \mid\left\{\tau_{\mathrm{h}}\right\},\left\{\beta_{\mathrm{h}}\right\}, \mathrm{V}_{\beta}$

Form $\beta_{h}^{*}$ by $C_{\tau h}^{-1} \beta_{\mathrm{h}}$.

$$
\begin{aligned}
& \bar{\beta} \sim \operatorname{MVN}\left(\overline{\mathrm{b}},\left(\left(\mathrm{V}_{\beta} / \mathrm{H}\right)^{-1}+(100 \mathrm{I})^{-1}\right)^{-1}\right) \\
& \left.\overline{\mathrm{b}}=\left(\left(\mathrm{V}_{\beta} / \mathrm{H}\right)^{-1}+(100 \mathrm{I})^{-1}\right)^{-1}\right)\left(\mathrm{V}_{\beta}{ }^{-1} \sum_{h=1}^{H} \beta_{h}^{*}+(100 \mathrm{I})^{-1}(0)\right) \overline{\mathrm{b}}
\end{aligned}
$$

4. Generate $\mathrm{V}_{\beta} \mid\left\{\tau_{\mathrm{h}}\right\},\left\{\beta_{\mathrm{h}}\right\}, \bar{\beta}$

Form $\beta_{h}^{*}$ by $C_{\tau h}^{-1} \beta_{\mathrm{h}}$.

$$
\mathrm{V}_{\beta} \sim \mathrm{IW}\left(v+\mathrm{H}, \Delta+\sum_{h=1}^{H}\left(\beta_{h}^{*}-\bar{\beta}\right)^{\prime}\left(\beta_{h}^{*}-\bar{\beta}\right)\right)
$$

5. Generate $\theta_{\mathrm{j}} \mid\left\{\tau_{\mathrm{hj}}\right\}$ for $\mathrm{j}=1, \ldots \mathrm{J}$

Let $\mathrm{s}_{\mathrm{hj}}=1$ if $\tau_{\mathrm{hj}}=1,0$ otherwise.

$$
\theta_{\mathrm{j}} \sim \operatorname{Binomial}\left(\mathrm{a}+\sum_{\mathrm{h}=1}^{\mathrm{H}} \mathrm{s}_{\mathrm{hj}}, \mathrm{H}-\sum_{\mathrm{h}=1}^{\mathrm{H}} \mathrm{s}_{\mathrm{hj}}+\mathrm{b}\right)
$$


6. Generate $\phi_{\mathrm{j}} \mid\left\{\lambda_{\mathrm{hj}}\right\}$ for $\mathrm{j}=1, \ldots \mathrm{J}$

Let $\mathrm{s}_{\mathrm{hj}}=1$ if $\lambda_{\mathrm{hj}}=1,0$ otherwise.

$$
\phi_{\mathrm{j}} \sim \operatorname{Binomial}\left(\mathrm{a}+\sum_{\mathrm{h}=1}^{\mathrm{H}} \mathrm{s}_{\mathrm{hj}}, \mathrm{H}-\sum_{\mathrm{h}=1}^{\mathrm{H}} \mathrm{s}_{\mathrm{hj}}+\mathrm{b}\right)
$$

\subsubsection{Simulation Results}

A simulation was conducted that demonstrates that the hybrid model is identified and the parameters can be recovered. Data was simulated for 500 individuals facing 10 initial multinomial choice tasks with four alternatives per task followed by a second set of 10 multinomial choice tasks, also with four alternatives. Explanatory variables for each alternative, $\mathrm{d}_{\mathrm{hijm}}$ and $\mathrm{x}_{\mathrm{hijk}}$, were generated from a uniform distribution. For identification, the explanatory variables for the fourth alternative in each choice task are set equal to 0 . Individual parameters $\lambda_{\mathrm{h}}, \tau_{\mathrm{h}}$, and $\beta_{\mathrm{h}}$ are drawn from the appropriate distributions and choices are simulated according to the model specified above.

The MCMC chain described above was used on the simulated data. Results are presented in table 3.3. All parameter values are recovered within $+/-2$ posterior standard deviations of the actual values.

The hybrid selection model is appropriate when two sets of choice data are available and respondents are assumed to be using a subset of product attributes across both choice tasks but a smaller subset is being used in one of the choice sets. Up to now, the variable selection models have been merely statistical models. In the next section, the threshold variable selection model is developed and it adds explanatory variables and reflects an extended model of choice. 


\subsection{Threshold Variable Selection Model}

The threshold variable selection model extends the previous models by adding structure and variables that explain why certain product attributes are important. The conceptual model is based on Fennell's model of action as described in chapter 2. In Fennell's extended choice model the explanatory variables are operationalized as concerns and interests. The threshold variable selection model only assumes that some appropriate explanatory variables are available, not necessarily concerns and interests, but retains other key elements of the behavioral process. Specifically, the model assumes only explanatory variables above some threshold matter and that there is a discrete mapping between the explanatory variables and the desired product attributes.

\subsubsection{Model Derivation}

The conceptual relationship between explanatory variables and selected product attributes is illustrated in Figure 3.3. In the figure, the bolded explanatory variables, $\mathrm{g}_{\mathrm{hp}}$, are those that are important to the individual in the particular choice situation. The arrows represent the mapping between the explanatory variables and the product attributes for that individual. Across choice situations, different explanatory variables may be important which, holding the map constant, would implicate different product attributes and potentially different choice outcomes. Note that in the figure, explanatory variable $6, \mathrm{~g}_{\mathrm{h} 6}$, is important to the individual, but does not map onto one of the existing product attributes. This is interpreted as unmet demand in the market. Unmet demand represents an opportunity for product manufacturers to introduce a new product or to 
influence the mapping between explanatory variables and product attributes through promotional efforts.

Two modeling devices are introduced to identify the important explanatory variables and the mapping between explanatory variables and product attributes. Let $\mathrm{I}\left(\mathrm{g}_{\mathrm{hp}}>\alpha_{\mathrm{h}}\right)=1$ if explanatory variable $\mathrm{p}$ is greater than the threshold $\alpha$ for household $\mathrm{h}$, otherwise $\mathrm{I}\left(\mathrm{g}_{\mathrm{hp}}>\alpha_{\mathrm{h}}\right)=0$. Here, $\alpha_{\mathrm{h}}$ is a household specific threshold to be estimated from the data and $\mathrm{g}_{\mathrm{hp}}$ is a measured variable with at least ordinal level properties. Let $\mathrm{g}_{\mathrm{h}}$ represent the vector of $\mathrm{p}$ explanatory variables and $\mathrm{I}\left(\mathrm{g}_{\mathrm{h}}>\alpha_{\mathrm{h}}\right)$ the corresponding vector of 0 's and 1's representing which explanatory variables exceed the threshold.

The mapping between explanatory variables and product attributes is represented by $\Lambda_{\mathrm{h}}$. Assume there are j product attributes and $\mathrm{p}$ concerns and interests. Then $\lambda_{\mathrm{hjp}}=1$ if explanatory variable $p$ maps to product attribute $j, \lambda_{\text {hip }}=0$ otherwise. Assume $j=p=4$, and consider:

$$
\left[\begin{array}{llll}
1 & 0 & 0 & 0 \\
0 & 1 & 0 & 0 \\
0 & 0 & 1 & 0 \\
0 & 0 & 0 & 1
\end{array}\right]=\Lambda_{\mathrm{h}} \quad \text { and } \quad\left[\begin{array}{llll}
0 & 1 & 0 & 0 \\
0 & 1 & 0 & 0 \\
0 & 0 & 0 & 0 \\
0 & 0 & 0 & 1
\end{array}\right]=\Lambda_{\mathrm{h}}^{*}
$$

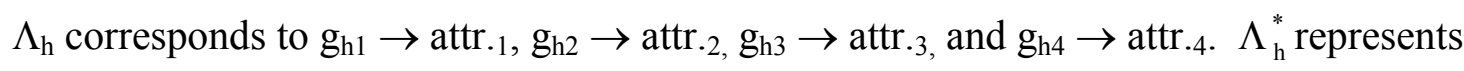
the following mapping: $\mathrm{g}_{\mathrm{h} 2} \rightarrow$ attr. $1, \mathrm{~g}_{\mathrm{h} 2} \rightarrow$ attr.2, and $\mathrm{g}_{\mathrm{h} 4} \rightarrow$ attr.4. Note that the $1^{\text {st }}$ and $3^{\text {rd }}$ column of $\Lambda_{\mathrm{h}}{ }^{*}$ are all 0 's indicating that $\mathrm{g}_{\mathrm{h} 1}$ and $\mathrm{g}_{\mathrm{h} 3}$ do not map onto product attributes. The $3^{\text {rd }}$ row of $\Lambda_{\mathrm{h}}^{*}$ is also all 0 's indicating that product attribute 3 does not address any of the explanatory variables for this respondent. Introduce the following 
additional notation. Let $\lambda_{\mathrm{hj}}$ ' represent the row vector formed by selecting the $\mathrm{j}$ 'th row from $\Lambda_{\mathrm{h}}$. From above, $\lambda_{\mathrm{h} 3^{\prime}}=\left[\begin{array}{llll}0 & 0 & 1 & 0\end{array}\right]$ and $\lambda_{\mathrm{h} 3}^{*}{ }^{\prime}=\left[\begin{array}{llll}0 & 0 & 0 & 0\end{array}\right]$.

Restrictions are necessary to control the dimensionality of the problem. Three types of restrictions on $\Lambda_{h}$ are tested. The first restriction requires that $\lambda_{h j}{ }^{\prime}$ be either a row of all 0 's, or to have a single 1 in the $\lambda_{\mathrm{hjj}}$ position. This is referred to as a diagonal map. The second restriction requires each $\lambda_{\mathrm{hj}}$ to contain at most, one non-zero element. Under this restriction, a product attribute can map to either one, or to no explanatory variable. However, a single explanatory variable may map to several product attributes. This is referred to as the row restricted map. A third restriction focuses on the columns of $\Lambda_{\mathrm{h}}$. In the column restricted map, each column may contain at most one non-zero element. This corresponds to an attribute being responsive to more than one explanatory variable. These relationships are summarized in figure 3.4.

The conceptual model is now operationalized in the threshold variable selection model. The derivation of the threshold variable selection model assumes two forms of data are available. The first type of data is a choice experiment where a respondent is exposed to all product attributes under study and makes choices among competing offerings. Similar to the previous variable selection models, in the first set of data it is assumed that the respondent is using all the product attributes presented either because it is a partial profile conjoint exercise or some other experimental manipulation ensures this outcome. In a second set of data, the same respondent is asked to make (or report on) brand choice decisions when facing the full array of product attributes, reflecting the choice the consumer actually faces in the market place. Concomitant measures of 
explanatory variables are taken with each full profile choice task. Variation in the explanatory variables across choices can be ensured through experimental manipulation, or through a natural experiment if actual brand choice data are available.

The model derivation considers two special aspects of the data consistent with the empirical application used in this thesis. The first set of data used to estimate the model is assumed to come from a rank-ordered conjoint task. Generalizing the model for a discrete choice task is straightforward. Second, the explanatory variables are measured on a 5 point Likert-type scale.

The model is represented as follows. Latent utility for the rank ordered conjoint task is represented as:

$$
\mathrm{U}_{\mathrm{him}}=\sum_{j=1}^{J} \beta_{\mathrm{hj}} \mathrm{d}_{\mathrm{hijm}}+\xi_{\text {him }}
$$

where $\mathrm{h}$ indexes the individual or household, $\mathrm{m}$ indexes the choice task, $\mathrm{i}$ indexes the alternative in choice task $\mathrm{m}$, and $\mathrm{j}$ indexes the product attributes. $\mathrm{d}_{\mathrm{hijm}}$ represents the level of the $\mathrm{j}^{\text {th }}$ product attribute for alternative $\mathrm{i}$, presented to household $\mathrm{h}$, in choice set $\mathrm{m}$. Latent utility for the full profile brand choice decision is represented as:

$$
\mathrm{Z}_{\mathrm{hik}}=\sum_{j=1}^{J} \beta_{\mathrm{hj}}\left[\lambda_{\mathrm{hj}}{ }^{\prime} \mathrm{I}\left(\mathrm{g}_{\mathrm{hk}}>\alpha_{\mathrm{h}}\right)\right] \mathrm{x}_{\mathrm{hijk}}+\varepsilon_{\mathrm{hik}}
$$

where the notation follows from above but with $\mathrm{k}$ choice occasions available for study. The representation for the row-restricted and diagonal models is used for illustration; changes in the algorithm for the column restricted model are described below. Recall that measures of the explanatory variables are available for all $\mathrm{k}$ choice occasions; hence $\mathrm{g}_{\mathrm{hk}}$ represents a vector of $\mathrm{p}$ explanatory variables measured on occasion $\mathrm{k}$. 
Equation (41) describes how each of the j product attributes is selected and used in brand evaluation. The product $\left[\lambda_{\mathrm{hj}} \mathrm{I}\left(\mathrm{g}_{\mathrm{hk}}>\alpha_{\mathrm{h}}\right)\right]$ equals 1 (a scalar) if the explanatory variable corresponding to product attribute $\mathrm{j}$ is above the threshold and "activated". The product $\left[\lambda_{\mathrm{hj}} \mathrm{I}\left(\mathrm{g}_{\mathrm{hk}}>\alpha_{\mathrm{h}}\right)\right]$ equals 0 if the appropriate explanatory variable is not activated, or if product attribute $\mathrm{j}$ does not map to any explanatory variable.

For the column restricted map, a slight modification is required. The product $\left[\lambda_{\mathrm{hj}}{ }^{\prime} \mathrm{I}\left(\mathrm{g}_{\mathrm{hk}}>\alpha_{\mathrm{h}}\right)\right]$ may equal 0,1 , or more than 1 if more than one activated explanatory variable maps on to attribute j. Equation (41) can be represented simply as:

$$
\mathrm{Z}_{\mathrm{hik}}=\sum_{j=1}^{J} \mathrm{G}\left(\beta_{\mathrm{hj}}, \Omega_{\mathrm{h}}, \alpha_{\mathrm{h}}\right) \mathrm{x}_{\mathrm{hijk}}+\varepsilon_{\mathrm{hi}}
$$

where $\Omega_{\mathrm{h}}$ represents individual level parameters, $\alpha_{\mathrm{h}}$ a threshold parameter, and the function $\mathrm{G}(\bullet)$ returns the value $\beta_{\mathrm{hj}}$ or $0 . \mathrm{G}(\bullet)$ equals $\beta_{\mathrm{hj}}$ if the explanatory variable(s) corresponding to product attribute $\mathrm{j}$ is activated, otherwise it equals 0 . Figure 3.4 illustrates the relationship between the various maps, $\Lambda_{\mathrm{h}}$, and the vector of indicator functions $\mathrm{I}\left(\mathrm{g}_{\mathrm{hk}}>\alpha_{\mathrm{h}}\right)$.

Assuming the $\xi$ and $\varepsilon$ are identically and independently distributed $\operatorname{EV}(0,1)$ leads to ordered logit and multinomial logit likelihoods for the observed rank orderings and brand choices, respectively. Following Chapman and Staelin (1982) the ordered logit likelihood for individual $\mathrm{h}$ for choice set $\mathrm{m}$ is represented as:

$$
\operatorname{Pr}\left(\mathrm{V}_{\mathrm{h} 1 \mathrm{~m}}>\mathrm{V}_{\mathrm{h} 2 \mathrm{~m}}>\ldots>\mathrm{V}_{\mathrm{hIm}}\right)=\prod_{i=1}^{I-1} \frac{\exp \left(\mathrm{d}_{\mathrm{him}}{ }^{\prime} \beta_{h}\right)}{\sum_{n=i}^{I} \exp \left(\mathrm{d}_{\mathrm{hnm}}{ }^{\prime} \beta_{h}\right)}
$$


where $V_{\text {him }}$ represents the rank order of alternatives. The multinomial logit choice probability for $\mathrm{y}_{\text {hik }}=1$ is represented as follows:

$$
\operatorname{Pr}\left(\mathrm{z}_{\text {hik }}>\mathrm{Z}_{\text {hnk }} \text { for all } \mathrm{N}\right)=\frac{\exp \left(\sum_{\mathrm{j}=1}^{\mathrm{J}} \beta_{\mathrm{hj}}\left[\lambda_{h j}{ }^{\prime} \mathrm{I}\left(\mathrm{g}_{\mathrm{hk}}>\alpha_{h}\right) \mathrm{x}_{\mathrm{hijk}}\right)\right.}{\sum_{n=1}^{N} \exp \left(\sum_{\mathrm{j}=1}^{\mathrm{J}} \beta_{\mathrm{hj}}\left[\lambda_{h j}{ }^{\prime} \mathrm{I}\left(\mathrm{g}_{\mathrm{hk}}>\alpha_{h}\right) \mathrm{x}_{\text {hnjk }}\right)\right.}
$$

Let $l_{l h}$ represent the product of equation (43) across the m ordered choice sets for individual $\mathrm{h}$ and $l_{2 h}$ represent the product of equation (44) across the $\mathrm{k}$ multinomial choice sets for individual $\mathrm{h}$. The likelihood for an individual is then represented by $l_{h}=l_{l h}$ $\times l_{2 h}$.

Heterogeneity for individual level model parameters is specified as follows:

$$
\begin{aligned}
& \beta_{\mathrm{h}} \sim \operatorname{MVN}\left(\bar{\beta}, \mathrm{V}_{\beta}\right) \\
& \alpha_{\mathrm{h}} \sim \operatorname{Multinomial}\left(\alpha_{4.5}, \alpha_{3.5}, \alpha_{3.5}, \alpha_{2.5}, \alpha_{1.5}, \alpha_{0.5}\right)
\end{aligned}
$$

where $\alpha_{4.5}$ represents the proportion of the sample with threshold value of 4.5. Recall that $\mathrm{g}_{\mathrm{hk}}$ is assumed to be measured on a 5 point scale giving rise to a discrete set of possible cut-off values. Extensions to the case when $g_{h k}$ is measured on a continuous scale are straightforward.

Different assumptions are made for $\Lambda_{\mathrm{h}}$ depending on the model. For the diagonal model, each row vector $\lambda_{\mathrm{hj}}$ ' can either have a 1 in the j'th position or be a vector of all 0 's. Therefore,

$$
\lambda_{\mathrm{hj}} \sim \operatorname{Bernoulli}\left(\theta_{\mathrm{j}}\right)
$$


where $\theta_{\mathrm{j}}$ is the probability the $\lambda_{\mathrm{hjj}}$ element equals 1 . For the row restricted model, $\lambda_{\mathrm{hj}}{ }^{\prime}$ can either have a 1 in the $\mathrm{j}^{\prime}$ th position, have a one in some other position, or be a vector of all 0's. Therefore,

$$
\lambda_{\mathrm{hj}} \sim \operatorname{Multinomial}\left(\theta_{\mathrm{jj}}, \theta_{\text {jother }}, \theta_{\text {jnot }}\right)
$$

where $\theta_{\mathrm{jj}}$ is the probability that the $\mathrm{jj}$ 'th element equals $1, \theta_{\mathrm{jother}}$ is the probability that some other element equals 1 , and $\theta_{\text {jnot }}$ is the probability that the vector is all 0 's. Note that this specification assumes the off-diagonal elements have an equal probability of being 1 and is a simplifying assumption. For the column restricted model, each column in $\Lambda_{\mathrm{h}}$ can have a 1 in the jj'th position, have a one in some other position, or be a column of all 0 's. Therefore,

$$
\lambda_{\mathrm{hj}} \sim \operatorname{Multinomial}\left(\theta_{\mathrm{jj}}, \theta_{\text {otherj }}, \theta_{\text {notj }}\right)
$$

where $\Lambda_{\mathrm{h}}$ is assumed to be a square $\mathrm{j} \times \mathrm{j}$ matrix and $\theta_{\mathrm{jj}}$ is the probability that the $\mathrm{jj}$ 'th element equals $1, \theta_{\text {otherj }}$ is the probability that some other element equals 1 , and $\theta_{\text {notj }}$ is the probability that the vector is all 0's. Again, off-diagonal elements are assumed to have equal probability for simplicity. Given enough data, the restriction on off-diagonal elements can be relaxed.

The model specification is completed by assuming prior distributions for the hyper-parameters.

$$
\begin{aligned}
& \bar{\beta} \sim \operatorname{MVN}(0,100 \mathrm{I}) \\
& \mathrm{V}_{\beta} \sim \operatorname{IW}(v, \Delta) \\
& \alpha * \sim \operatorname{Dirichlet}(\mathrm{d}) \\
& \theta_{\mathrm{j}} \sim \operatorname{Beta}(\mathrm{a}, \mathrm{b}) \text { for the diagonal model }
\end{aligned}
$$


$\theta_{\mathrm{j}^{*}} \sim \operatorname{Dirichlet}(\mathrm{c})$ for the row restricted model

$\theta_{*_{\mathrm{j}}} \sim \operatorname{Dirichlet}(\mathrm{f})$ for the column restricted model

where IW is the inverted Wishart distribution with $v=\mathrm{J}+8$, and $\Delta=v \mathrm{I} . \quad \alpha *$ is a vector of length 5 corresponding to the discrete values $\alpha_{\mathrm{h}}$ can assume and $\mathrm{d}$ is a vector of length 5 with each element set equal to 5 . For the diagonal model, $a=1$ and $b=1$. For the row and column restricted models $\theta_{\mathrm{j}^{*}}$ and $\theta_{*_{\mathrm{j}}}$ are each of length 3 and $\mathrm{c}$ and $\mathrm{f}$ are corresponding vectors each with elements equal to 3 .

\subsubsection{Estimation Algorithm}

This section details the estimation algorithm for the threshold variable selection model. The following steps describe an MCMC chain for the model (43)-(51) with the posterior distribution of all model parameters as the stationary distribution.

1. Generate $\beta_{\mathrm{h}} \mid \Lambda_{\mathrm{h}}, \alpha_{\mathrm{h}}, \bar{\beta}, \mathrm{V}_{\beta}, \mathrm{y}_{\mathrm{h}}, \mathrm{X}_{\mathrm{h}}, \mathrm{D}_{\mathrm{h}}, \mathrm{V}_{\mathrm{h}}$ for $\mathrm{h}=1, \ldots, \mathrm{H}$

A random walk Metropolis-Hastings algorithm is used to draw from the posterior

distribution. A candidate vector $\beta_{h}^{(n)}$ is formed as $\beta_{h}^{(n)}=\beta_{h}^{(o)}+\zeta$ where $\zeta \sim \operatorname{MVN}(0, \mathrm{wI})$ and $\mathrm{w}$ is chosen to ensure a $50 \%$ acceptance rate. $\beta_{h}^{(o)}$ represents the previous, or "old" draw of $\beta_{\mathrm{h}}$. The new parameter is accepted with probability:

$$
\operatorname{Pr}(\text { accept })=\min \left(\frac{l_{h}\left(\beta_{h}^{(n)}\right) \times \exp \left(-\frac{1}{2}\left(\beta_{h}^{(n)}-\bar{\beta}\right)^{\prime} \mathrm{V}_{\beta}^{-1}\left(\beta_{h}^{(n)}-\bar{\beta}\right)\right)}{l_{h}\left(\beta_{h}^{(o)}\right) \times \exp \left(-\frac{1}{2}\left(\beta_{h}^{(o)}-\bar{\beta}\right)^{\prime} \mathrm{V}_{\beta}^{-1}\left(\beta_{h}^{(o)}-\bar{\beta}\right)\right)}, 1\right)
$$


2. Generate $\lambda_{\mathrm{hj}} \mid \lambda_{\mathrm{h}-\mathrm{j}}, \beta_{\mathrm{h}}, \alpha_{\mathrm{h}}, \theta_{\mathrm{j}}, \mathrm{y}_{\mathrm{h}}, \mathrm{X}_{\mathrm{h}}$ for $\mathrm{j}=1, \ldots, \mathrm{J}$ and $\mathrm{h}=1, \ldots, \mathrm{H}$

An independence chain Metropolis-Hastings algorithm is used with the prior distribution as the generating distribution. Draw $\lambda_{h j}^{(n)}$ from the appropriate distribution according to the specified model. If $\lambda_{h j}^{(n)}=$ "other" for the row or column restricted models, then the indicator is assigned at random to a non-diagonal element in row j. Accept the new draw with probability:

$$
\operatorname{Pr}(\text { accept })=\min \left(\frac{l_{2 h}\left(\lambda_{h}^{(n)}\right)}{l_{2 h}\left(\lambda_{h}^{(o)}\right)}, 1\right)
$$

Note two items: first, since the prior, in this case the distribution heterogeneity, is used to generate the candidate, it is not used to evaluate the acceptance probability. And second, only the likelihood from the second half of the data is used to evaluate parameters that are unique to the threshold variable selection model.

\section{Generate $\alpha_{\mathrm{h}} \mid \beta_{\mathrm{h}}, \Lambda_{\mathrm{h}}, \alpha_{*}, \mathrm{y}_{\mathrm{h}}, \mathrm{X}_{\mathrm{h}}$ for $\mathrm{h}=1, \ldots, \mathrm{H}$}

Since $\alpha_{h}$ takes on one of a discrete set of values, a griddy Gibbs sampler is used. Let $\mathrm{i}$ index the 5 possible values $\alpha_{\mathrm{h}}$ may equal and $\alpha_{(\mathrm{i})}$ the corresponding probability taken from $\alpha *$ Draw $\alpha_{h}^{(i)}$ with probability:

$$
\operatorname{Pr}\left(\alpha_{h}^{(i)}\right)=\frac{l_{2 h}\left(\alpha_{h}^{(i)}\right) \times \alpha_{(i)}}{\sum_{i=1}^{5} l_{2 h}\left(\alpha_{h}^{(i)}\right) \times \alpha_{(i)}}
$$


4. Generate $\bar{\beta} \mid\left\{\beta_{\mathrm{h}}\right\}, \mathrm{V}_{\beta}$

$$
\begin{aligned}
& \bar{\beta} \sim \operatorname{MVN}\left(\overline{\mathrm{b}},\left(\left(\mathrm{V}_{\beta} / \mathrm{H}\right)^{-1}+(100 \mathrm{I})^{-1}\right)^{-1}\right) \\
& \left.\overline{\mathrm{b}}=\left(\left(\mathrm{V}_{\beta} / \mathrm{H}\right)^{-1}+(100 \mathrm{I})^{-1}\right)^{-1}\right)\left(\mathrm{V}_{\beta}^{-1} \sum_{h=1}^{H} \beta_{h}+(100 \mathrm{I})^{-1}(0)\right)
\end{aligned}
$$

5. Generate $\mathrm{V}_{\beta} \mid\left\{\beta_{\mathrm{h}}\right\}, \bar{\beta}$

$$
\mathrm{V}_{\beta} \sim \operatorname{IW}\left(v+\mathrm{H}, \Delta+\sum_{h=1}^{H}\left(\beta_{h}-\bar{\beta}\right)^{\prime}\left(\beta_{h}-\bar{\beta}\right)\right)
$$

6. Generate $\alpha *\left\{\alpha_{\mathrm{h}}\right\}$

Define new variables: $\mathrm{s}_{\mathrm{h} 1}=1$ if $\alpha_{\mathrm{h}}=4.5,0$ otherwise; $\mathrm{s}_{\mathrm{h} 2}=1$ if $\alpha_{\mathrm{h}}=3.5,0$ otherwise; etc.

Then,

$$
\alpha_{*} \sim \operatorname{Dirichlet}\left(\sum_{h=1}^{H} s_{h 1}+d_{1}, \ldots, \sum_{h=1}^{H} s_{h 5}+d_{5}\right)
$$

See Allenby, Arora, and Ginter (1998) for additional details on drawing from a Dirichlet.

\section{Generate $\theta_{\mathrm{j}} \mid\left\{\lambda_{\mathrm{hj}}\right\}$ for $\mathrm{j}=1, \ldots, \mathrm{J}$}

For the diagonal model, let $\mathrm{s}_{\mathrm{hj}}=1$ if $\lambda_{\mathrm{hjj}}=1,0$ otherwise. Then,

$$
\theta_{\mathrm{j}} \sim \operatorname{Binomial}\left(\mathrm{a}+\sum_{\mathrm{h}=1}^{\mathrm{H}} \mathrm{s}_{\mathrm{hj}}, \mathrm{H}-\sum_{\mathrm{h}=1}^{\mathrm{H}} \mathrm{s}_{\mathrm{hj}}+\mathrm{b}\right)
$$


For the row restricted model, let $\mathrm{s}_{\mathrm{hj} 1}=1$ if $\lambda_{\mathrm{hjj}}=1,0$ otherwise; let $\mathrm{s}_{\mathrm{hj} 2}=1$ if some other element of $\lambda_{\mathrm{hj}}=1,0$ otherwise; and let $\mathrm{s}_{\mathrm{hj} 3}=1$ if $\lambda_{\mathrm{hj}}$ is a vector of all 0 's, $\mathrm{s}_{\mathrm{hj} 3}=0$ otherwise. Then

$$
\theta_{\mathrm{j}^{*}} \sim \operatorname{Dirichlet}\left(\sum_{h=1}^{H} s_{h j 1}+c_{1}, \ldots, \sum_{h=1}^{H} s_{h j 3}+c_{3}\right)
$$

For the column restricted model, $\theta_{* \mathrm{j}}$ is drawn analogous to $\theta_{\mathrm{j} *}$.

\subsubsection{Simulation Results}

This section reports on a series of simulation studies completed for the threshold variable selection model. Tables 3.4 to 3.6 show results from simulations when multiple brand choice observations are available. For each brand choice, concomitant measures of the explanatory variables, $\mathrm{g}_{\mathrm{hk}}$, are also available. Consistent with the empirical study, the explanatory variables will be referred to as concerns and interests or c/i's. Data was simulated assuming 500 individuals who completed 10 rank-ordered choice tasks and 30 full profile brand choice tasks using the threshold variable selection model described in section 3.4.1. Each brand had 5 product attributes (for both the ordered and multinomial choice tasks) with values generated from a random uniform distribution; each choice set consisted of four brands. For identification, the predictor variables for the fourth alternative are set to 0 in each choice set. For each of the 30 multinomial choice tasks for each individual, a vector of $6 \mathrm{c} / \mathrm{i}$ 's measured on a 5 point scale was generated. Individual values of $\beta_{\mathrm{h}}, \Lambda_{\mathrm{h}}$, and $\alpha_{\mathrm{h}}$ were generated according to the specified distributions, and simulated choices generated. 
Tables 3.4 to 3.6 indicate that all parameter values are recovered for the diagonal, row restricted, and column restricted threshold variable selection models. The posterior distribution of $\mathrm{V}_{\beta}$ for each model was also recovered but is not displayed to conserve space. Two derived measures of particular interest to managers are also displayed. "Attributes used" is simply a tally of $\lambda_{\mathrm{hj}}{ }^{\prime} \mathrm{I}\left(\mathrm{g}_{\mathrm{hk}}>\alpha_{\mathrm{h}}\right)$ for each attribute, $\mathrm{j}$, across respondents and choice sets. An "unmet $\mathrm{c} / \mathrm{i}$ " is measured as $\mathrm{g}_{\mathrm{hpk}}>\alpha_{\mathrm{h}}$ and the $\mathrm{c} / \mathrm{i}$ is not mapped onto an attribute that is used in the brand choice. Unmet c/i's represent an opportunity for marketing managers to create new products or to reposition existing products to better meet the needs of consumers.

In the remaining simulations, it is assumed that only one brand choice observation with concomitant measures of $\mathrm{c} / \mathrm{i}$ 's for each person is available for study. This is consistent with the empirical study described in the second half of chapter 4 . When only one brand choice observation is available, then $\Lambda_{\mathrm{h}}$ and $\alpha_{\mathrm{h}}$ are not both well identified. This is illustrated in figure 3.5. By systematically changing $\Lambda_{\mathrm{h}}$ and $\alpha_{\mathrm{h}}$ the same set of attributes will be selected, yielding identical values for the likelihood. As demonstrated in the previous set of simulations, when multiple observations per respondent are available, all the parameter values are well identified.

Two methods are investigated for dealing with the poorly identified parameters. In the first method, it is assumed that consumers are homogeneous in their use of the 5 point Likert scale used to measure the relative importance of $c / i$ 's. This assumption means that a common threshold value, $\alpha$ can either be estimated from the data, or assumed a priori. It is common in applied marketing research studies to look at the "Top two box" 
measure as an indicator of "importance". The "Top two box" measure is consistent with $\alpha=3.5$.

This second set of simulations assumes that only one brand choice per individual is available for study and consumers are homogeneous in their use of the 5 point likert scale for the c/i's. 500 individuals are assumed to complete 10 rank ordered tasks and a single brand choice. Five product attributes are used and a single set of $5 \mathrm{c} / \mathrm{i}$ 's is generated for each individual. Heterogeneity is assumed for $\beta$ and $\Lambda$. Tables 3.7 to 3.9 indicate that all the parameters are identified and can be recovered within $+/-2$ posterior standard deviations of the actual values. With only one brand choice per individual, however, the posterior standard deviations for the estimates of the "attributes used", "unmet c/i's", and the mapping between c/i's and attributes (the $\theta$ 's) increases compared to the simulation where multiple choices are observed.

The second method for dealing with the poorly identified parameters is to simply ignore the problem and focus inference on the parameters that are well identified. As long as the posterior is proper, Bayesian methods can still be used to estimate the model. For a discussion and illustration of this topic see Edwards and Allenby (2003). For the third set of simulations, it is assumed that consumers are heterogeneous in their use of the rating scale and $\alpha_{\mathrm{h}}$ is estimated, but only results for the identified parameters are reported. Tables 3.10 to 3.12 show that the distribution of $\beta$ 's and "attributes used" can be recovered when there is scale use heterogeneity.

The threshold variable selection model provides a behavioral explanation of why certain product attributes are used by a consumer. As such, it extends the heterogeneous variable selection and pooled variable selection models presented earlier. The derivation 
of the threshold variable selection model relies on the model of action by Fennell as its conceptual underpinning. However, other models of action or extended models of choice may provide the explanatory variables used in other applications of the threshold variable selection model.

\subsection{Summary}

This chapter introduces models for individual level variable selection for use in discrete choice and rank ordered choice data. The heterogeneous variable selection model extends the previous literature on Bayesian variable selection to identify at the individual level the most promising subset of variables. The pooled variable selection model uses data from separate response contexts and assumes only a subset of variables are used by each individual in one of the contexts. Examples of separate contexts include partial and full profile choice experiments or choice experiments and actual market place transactions. A hybrid model is introduced that incorporates contextual variation into the heterogeneous variable selection model. Finally, the threshold variable selection model incorporates explanatory variables and an extended model of choice to determine which variables are selected. Tractable estimation algorithms are detailed and simulation results

demonstrating that the models are identified and that the parameters can be recovered are presented.

The next chapter presents results from two empirical applications of the models introduced in this chapter. The first focuses on physicians' preferences for a medical device in a partial profile and full profile discrete choice study. The second study 
combines experimental and self-reported choices on consumers' preferences for toothpaste. 


\begin{tabular}{|c|c|c|c|c|}
\cline { 2 - 5 } \multicolumn{1}{c|}{} & $\begin{array}{c}\text { Previous Bayesian } \\
\text { Variable Selection }\end{array}$ & $\begin{array}{c}\text { Heterogeneous } \\
\text { Variable Selection }\end{array}$ & $\begin{array}{c}\text { Pooled Variable } \\
\text { Selection }\end{array}$ & $\begin{array}{c}\text { Threshold Variable } \\
\text { Selection }\end{array}$ \\
\hline $\begin{array}{c}\text { Which product } \\
\text { attributes are } \\
\text { important }\end{array}$ & $\checkmark$ & $\checkmark$ & $\checkmark$ & $\checkmark$ \\
\hline $\begin{array}{c}\text { Who are they } \\
\text { important to }\end{array}$ & $\checkmark$ & $\checkmark$ & $\checkmark$ \\
\hline $\begin{array}{c}\text { When are they } \\
\text { important }\end{array}$ & & $\checkmark$ & $\checkmark$ \\
\hline $\begin{array}{c}\text { Why are they } \\
\text { important }\end{array}$ & & & & $\checkmark$ \\
\hline
\end{tabular}

Figure 3.1 Comparison of models and substantive issues. 


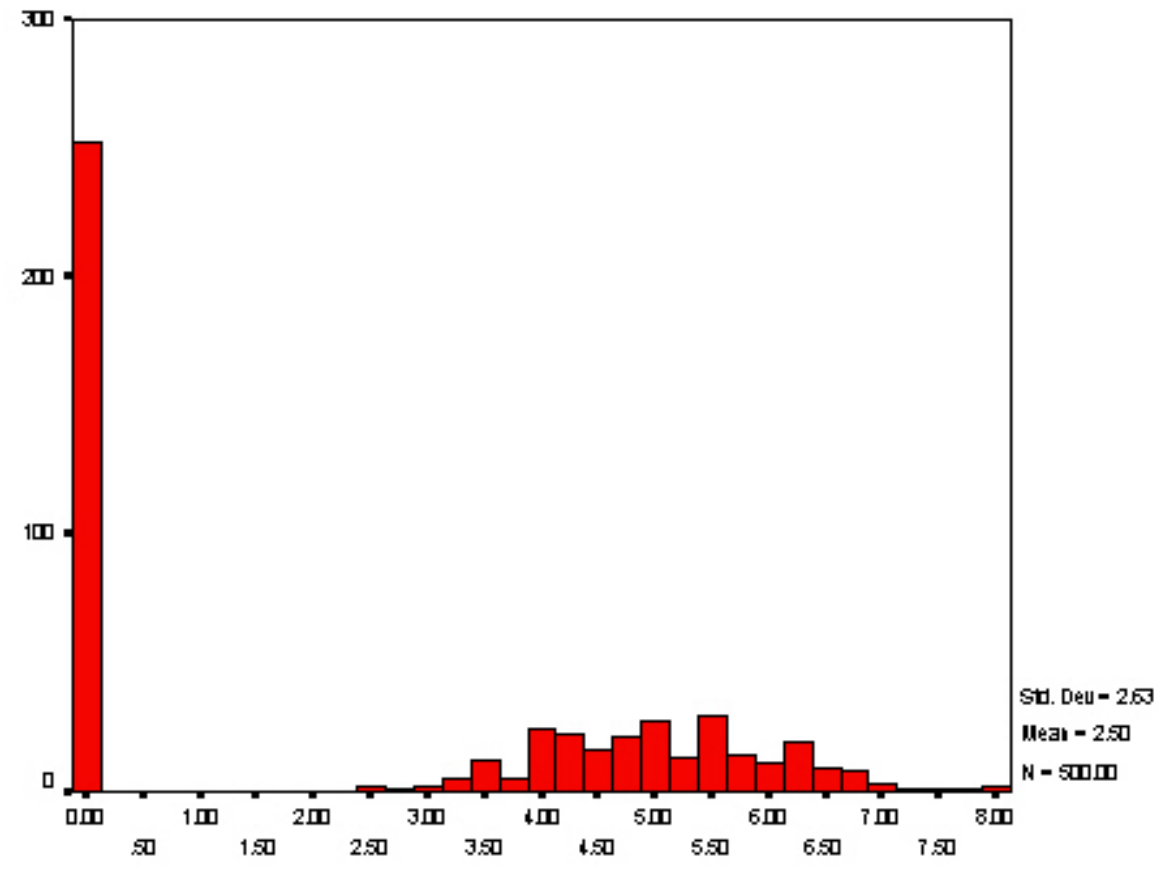

BETA1

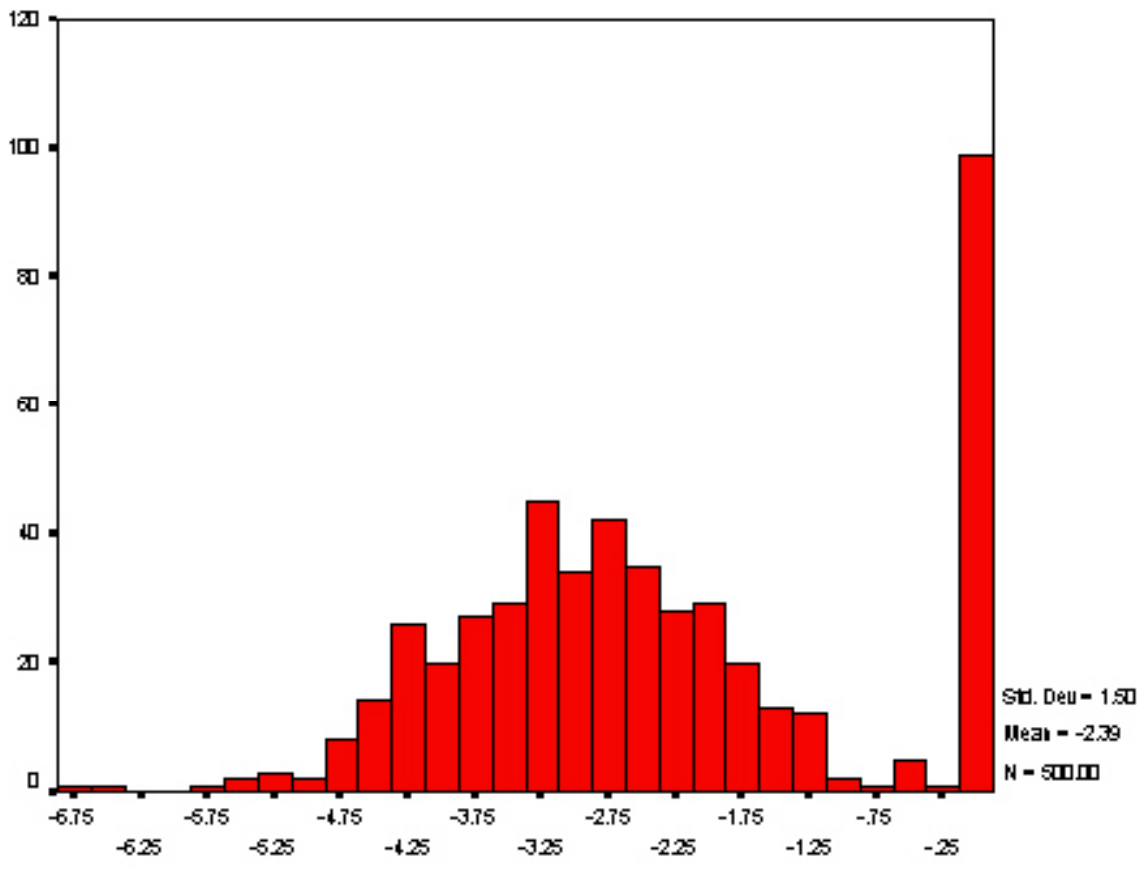

BETA4

Figure 3.2 Distributions of heterogeneity with mass centered at 0 and away from 0. 
Simulation Results: Heterogeneous Variable Selection Model

\begin{tabular}{|c|c|c|c|c|c|c|c|}
\hline \multicolumn{3}{|c|}{ Distribution of $\beta$} & \multicolumn{5}{|c|}{ Recovery of $\theta$ 's } \\
\hline & Actual & Posterior & Posterior & & Actual & Posterior & Posterior \\
\hline & Mean & Mean & Std. Dev. & & Value & Mean & Std. Dev. \\
\hline$\beta_{1}$ & 5.05 & 5.15 & $(.25)$ & $\theta_{1}$ & 0.50 & 0.48 & $(.03)$ \\
\hline$\beta_{2}$ & 4.05 & 3.98 & $(.14)$ & $\theta_{2}$ & 0.91 & 0.93 & $(.03)$ \\
\hline$\beta_{3}$ & 3.08 & 2.71 & $(.22)$ & $\theta_{3}$ & 0.71 & 0.78 & $(.06)$ \\
\hline$\beta_{4}$ & -2.98 & -2.81 & $(.27)$ & $\theta_{4}$ & 0.80 & 0.80 & $(.07)$ \\
\hline$\beta_{5}$ & -4.05 & -3.89 & $(.24)$ & $\theta_{5}$ & 0.58 & 0.62 & $(.04)$ \\
\hline
\end{tabular}

\begin{tabular}{ccccc} 
Recovery of & \multicolumn{5}{c}{$\mathbf{V}_{\beta}$ - posterior means } \\
1.47 & -0.04 & 0.04 & -0.06 & 0.12 \\
-0.04 & 1.03 & 0.08 & -0.02 & -0.08 \\
0.04 & 0.08 & 1.09 & 0.12 & -0.21 \\
-0.06 & -0.02 & 0.12 & 1.10 & -0.03 \\
0.12 & -0.08 & -0.21 & -0.03 & 1.35
\end{tabular}

Actual covariance matrix equal to the identity matrix. All posterior means are within $+/-2$ posterior std. dev. of actual values.

Parameter estimates assuming a standard MVN prior distribution

Distribution of $\beta$ 's

$\begin{array}{ccc} & \begin{array}{c}\text { Posterior } \\ \text { Mean }\end{array} & \begin{array}{c}\text { Posterior } \\ \text { Std. Dev. }\end{array} \\ \beta_{1} & 2.43 & (.15) \\ \beta_{2} & 3.66 & (.11) \\ \beta_{3} & 2.08 & (.11) \\ \beta_{4} & -2.23 & (.11) \\ \beta_{5} & -2.40 & (.13)\end{array}$

$\begin{array}{ccccc}\text { Estimate of } \mathbf{V}_{\boldsymbol{\beta}} \text { - posterior means } & & \\ 4.60 & 0.67 & 0.33 & 0.16 & -0.20 \\ 0.67 & 2.86 & 0.05 & 0.15 & -0.92 \\ 0.33 & 0.05 & 1.32 & -0.10 & 0.10 \\ 0.16 & 0.15 & -0.10 & 1.30 & 0.00 \\ -0.20 & -0.92 & 0.10 & 0.00 & 2.85\end{array}$

Table 3.1 Simulation results for heterogeneous variable selection model compared to standard model. 
Simulation Results: Pooled Variable Selection Model

\begin{tabular}{|c|c|c|c|c|c|c|c|}
\hline \multicolumn{3}{|c|}{ Distribution of $\beta$} & \multicolumn{5}{|c|}{ Recovery of $\theta$ 's } \\
\hline & Actual & Posterior & Posterior & & Actual & Posterior & Posterior \\
\hline & Mean & Mean & Std. Dev. & & Value & Mean & Std. Dev. \\
\hline$\beta_{1}$ & 1.94 & 1.95 & $(.08)$ & $\theta_{1}$ & 0.87 & 0.87 & $(.04)$ \\
\hline$\beta_{2}$ & 1.48 & 1.54 & $(.07)$ & $\theta_{2}$ & 0.81 & 0.74 & $(.05)$ \\
\hline$\beta_{3}$ & 0.50 & 0.46 & $(.07)$ & $\theta_{3}$ & 0.71 & 0.69 & $(.09)$ \\
\hline$\beta_{4}$ & -0.56 & -0.54 & $(.06)$ & $\theta_{4}$ & 0.58 & 0.57 & $(.08)$ \\
\hline$\beta_{5}$ & -0.97 & -1.03 & $(.07)$ & $\theta_{5}$ & 0.46 & 0.36 & $(.08)$ \\
\hline
\end{tabular}

$\begin{array}{ccccc}\text { Recovery of } & \mathbf{V}_{\beta} \text { - posterior means } & & \\ 1.45 & 0.11 & 0.02 & 0.12 & -0.01 \\ 0.11 & 1.30 & -0.23 & -0.04 & -0.10 \\ 0.02 & -0.23 & 1.81 & 0.02 & -0.10 \\ 0.12 & -0.04 & 0.02 & 1.39 & 0.07 \\ -0.01 & -0.10 & -0.10 & 0.07 & 1.50\end{array}$

Actual covariance matrix equal to 1.5I. All posterior means are within $+/-2$ posterior std. dev. of actual values.

Table 3.2 Simulation results for the pooled variable selection model. 


\section{Simulation Results: Hybrid Variable Selection Model}

\begin{tabular}{|c|c|c|c|c|c|c|c|}
\hline \multicolumn{3}{|c|}{ Distribution of $\beta$} & \multicolumn{5}{|c|}{ Recovery of $\theta$ 's } \\
\hline & Actual & Posterior & Posterior & & Actual & Posterior & Posterior \\
\hline & Mean & Mean & Std. Dev. & & Value & Mean & Std. Dev. \\
\hline$\beta_{1}$ & 2.96 & 2.91 & $(.17)$ & $\theta_{1}$ & 0.51 & 0.49 & $(.04)$ \\
\hline$\beta_{2}$ & 2.00 & 1.90 & $(.15)$ & $\theta_{2}$ & 0.90 & 0.94 & $(.06)$ \\
\hline$\beta_{3}$ & 1.02 & 0.85 & $(.17)$ & $\theta_{3}$ & 0.71 & 0.73 & $(.13)$ \\
\hline$\beta_{4}$ & -2.00 & -2.11 & $(.19)$ & $\theta_{4}$ & 0.77 & 0.73 & $(.07)$ \\
\hline$\beta_{5}$ & -2.97 & -2.73 & $(.19)$ & $\theta_{5}$ & 0.58 & 0.60 & $(.05)$ \\
\hline
\end{tabular}

\begin{tabular}{cccc}
\multicolumn{2}{c}{$\begin{array}{c}\text { Recovery of } \phi \\
\text { Actual } \\
\text { Value }\end{array}$} & $\begin{array}{c}\text { Posterior } \\
\text { Mean }\end{array}$ & $\begin{array}{c}\text { Posterior } \\
\text { Std. Dev. }\end{array}$ \\
$\phi_{1}$ & 0.92 & 0.88 & $(.04)$ \\
$\phi_{2}$ & 0.51 & 0.56 & $(.04)$ \\
$\phi_{3}$ & 0.61 & 0.68 & $(.11)$ \\
$\phi_{4}$ & 0.71 & 0.68 & $(.05)$ \\
$\phi_{5}$ & 0.81 & 0.83 & $(.05)$
\end{tabular}

$\begin{array}{ccccc}\text { Recovery of } & \mathbf{V}_{\beta} \text { - posterior means } & & \\ 0.89 & -0.16 & -0.01 & -0.07 & 0.08 \\ -0.16 & 1.15 & 0.18 & 0.04 & 0.01 \\ -0.01 & 0.18 & 0.96 & 0.07 & 0.16 \\ -0.07 & 0.04 & 0.07 & 0.89 & 0.04 \\ 0.08 & 0.01 & 0.16 & 0.04 & 1.24\end{array}$

Actual covariance matrix equal to the identity matrix. All posterior means are within $+/-2$ posterior std. dev. of actual values.

Table 3.3 Simulation results from the hybrid variable selection model. 


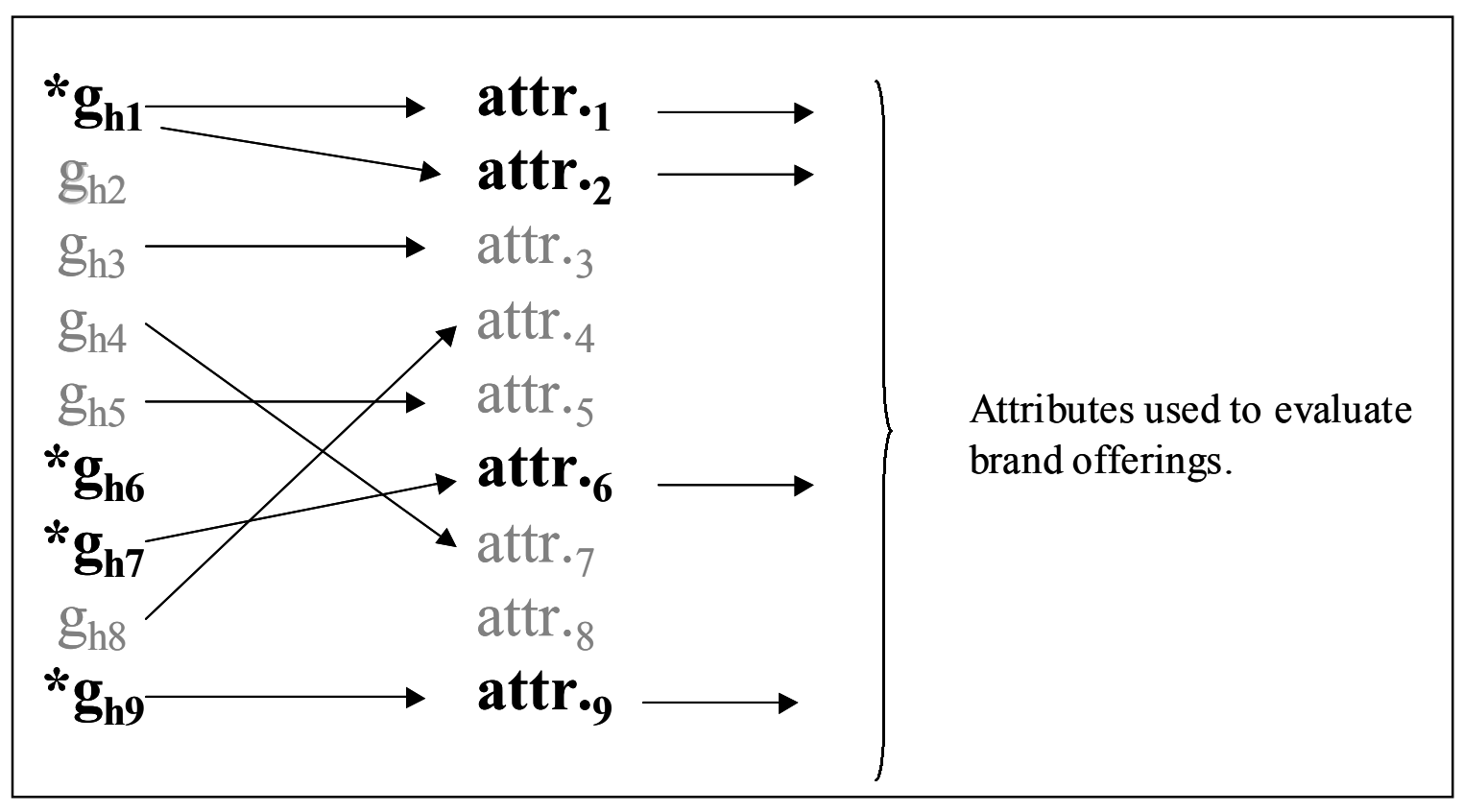

Figure 3.3 Activated explanatory variables and the map to product attributes. 


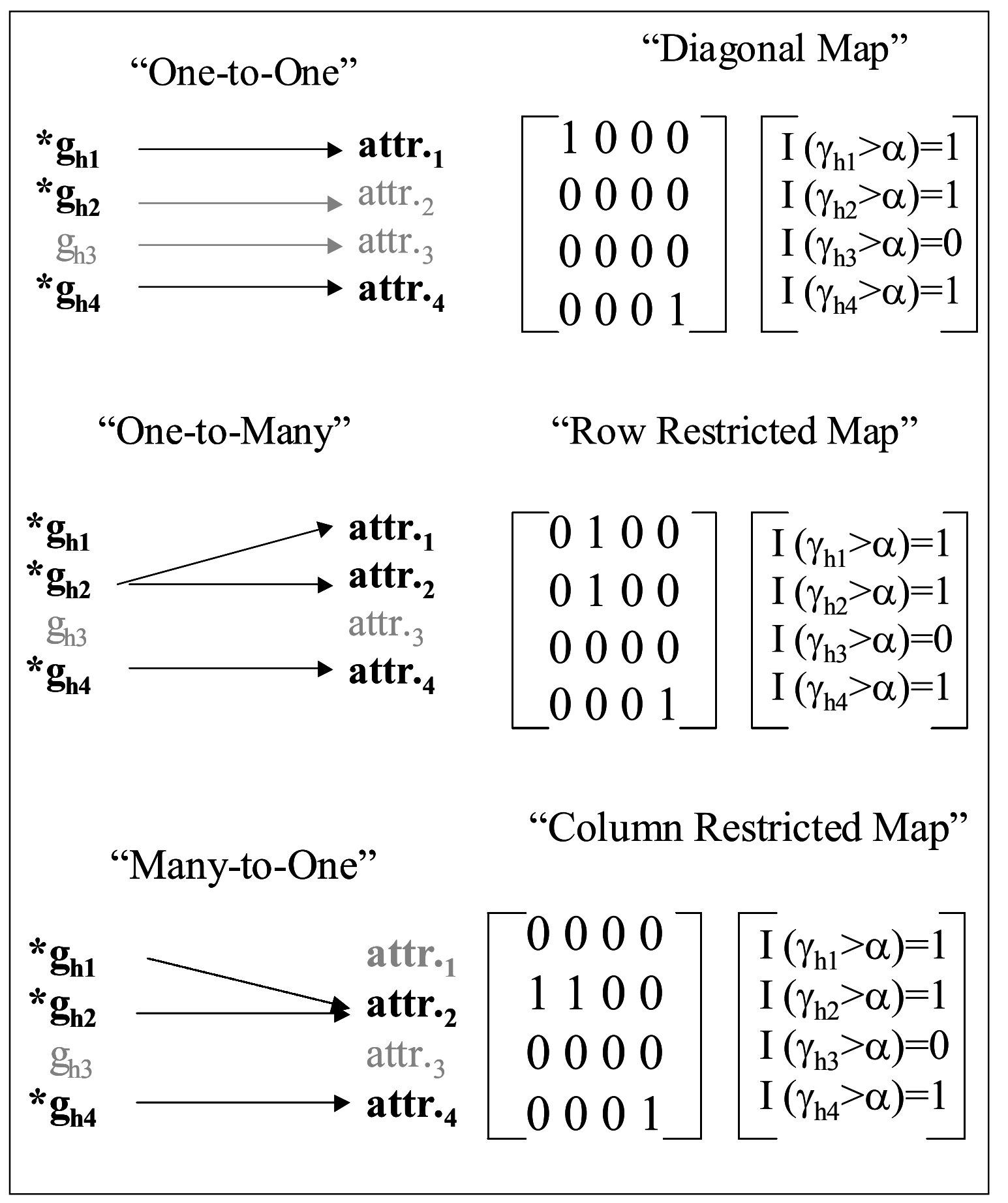

Figure 3.4 Different maps, $\Lambda_{\mathrm{h}}$, and vectors of indicator functions $\mathrm{I}\left(\mathrm{g}_{\mathrm{h}}>\alpha\right)$. 
Simulation results, Diagonal: One-to-one Mapping, Multiple Observations

Distribution of $\beta$ 's

$\begin{array}{lccc} & \begin{array}{c}\text { Actual } \\ \text { Mean }\end{array} & \begin{array}{c}\text { Posterior } \\ \text { Mean }\end{array} & \begin{array}{c}\text { Posterior } \\ \text { Std. Dev. }\end{array} \\ \beta_{1} & 2.04 & 2.08 & (.06) \\ \beta_{2} & 1.42 & 1.43 & (.06) \\ \beta_{3} & 0.51 & 0.52 & (.06) \\ \beta_{4} & -0.41 & -0.41 & (.06) \\ \beta_{5} & -0.94 & -0.97 & (.06)\end{array}$

Attributes Used

$\begin{array}{lccc} & \text { Actual } & \begin{array}{c}\text { Posterior } \\ \text { Mean }\end{array} & \begin{array}{c}\text { Posterior } \\ \text { Std. Dev. }\end{array} \\ \mathrm{x}_{1} & 0.54 & 0.54 & (.01) \\ \mathrm{x}_{2} & 0.44 & 0.45 & (.01) \\ \mathrm{x}_{3} & 0.41 & 0.42 & (.01) \\ \mathrm{x}_{4} & 0.34 & 0.36 & (.01) \\ \mathrm{x}_{5} & 0.29 & 0.28 & (.01)\end{array}$

\section{Distribution $\alpha$ 's}

$\begin{array}{llcc} & \text { Actual } & \begin{array}{c}\text { Posterior } \\ \text { Mean }\end{array} & \begin{array}{c}\text { Posterior } \\ \text { Std. Dev. }\end{array} \\ \alpha=4.5 & 0.10 & 0.11 & (.02) \\ \alpha=3.5 & 0.30 & 0.29 & (.02) \\ \alpha=2.5 & 0.30 & 0.29 & (.02) \\ \alpha=1.5 & 0.16 & 0.19 & (.02) \\ \alpha=0.5 & 0.13 & 0.12 & (.02)\end{array}$

\begin{tabular}{|c|c|c|}
\hline \multicolumn{3}{|c|}{ Unmet C/I's } \\
\hline & Posterior & Posterior \\
\hline Actual & Mean & Std. Dev. \\
\hline 0.05 & 0.05 & $(.01)$ \\
\hline 0.14 & 0.13 & $(.01)$ \\
\hline 0.18 & 0.16 & $(.01)$ \\
\hline 0.24 & 0.21 & $(.02)$ \\
\hline 0.30 & 0.30 & $(.01)$ \\
\hline 0.59 & 0.58 & $(.01)$ \\
\hline
\end{tabular}

\begin{tabular}{|c|c|c|}
\hline \multicolumn{3}{|c|}{ Recovery of Mapping from C/I's to Attributes } \\
\hline Actual & Posterior & Posterior \\
\hline$\theta_{\mathrm{jj}}$ & Mean & Std. Dev. \\
\hline 0.91 & 0.92 & $(.01)$ \\
\hline 0.75 & 0.78 & $(.02)$ \\
\hline 0.70 & 0.72 & $(.03)$ \\
\hline 0.58 & 0.63 & $(.03)$ \\
\hline 0.50 & 0.49 & $(.03)$ \\
\hline
\end{tabular}

Table 3.4 Simulation results for the threshold selection model, diagonal: one-to-one mapping, multiple observations. 
Simulation results, Row: One-to-many Mapping, Multiple Observations

Distribution of $\beta$ 's

$\begin{array}{lccc} & \begin{array}{c}\text { Actual } \\ \text { Mean }\end{array} & \begin{array}{c}\text { Posterior } \\ \text { Mean }\end{array} & \begin{array}{c}\text { Posterior } \\ \text { Std. Dev. }\end{array} \\ \beta_{1} & 2.06 & 2.05 & (.06) \\ \beta_{2} & 1.46 & 1.45 & (.06) \\ \beta_{3} & 0.55 & 0.56 & (.06) \\ \beta_{4} & -0.50 & -0.51 & (.06) \\ \beta_{5} & -0.92 & -0.92 & (.06)\end{array}$

Attributes Used

$\begin{array}{lccc} & \text { Actual } & \begin{array}{c}\text { Posterior } \\ \text { Mean }\end{array} & \begin{array}{c}\text { Posterior } \\ \text { Std. Dev. }\end{array} \\ \mathrm{x}_{1} & 0.52 & 0.53 & (.01) \\ \mathrm{x}_{2} & 0.49 & 0.49 & (.01) \\ \mathrm{x}_{3} & 0.41 & 0.41 & (.01) \\ \mathrm{x}_{4} & 0.33 & 0.32 & (.01) \\ \mathrm{x}_{5} & 0.28 & 0.28 & (.01)\end{array}$

$\begin{array}{llcc}\text { Distribution } \alpha \text { 's } & & \\ & \text { Actual } & \begin{array}{c}\text { Posterior } \\ \text { Mean }\end{array} & \begin{array}{c}\text { Posterior } \\ \text { Std. Dev. }\end{array} \\ \alpha=4.5 & 0.09 & 0.10 & (.02) \\ \alpha=3.5 & 0.29 & 0.27 & (.02) \\ \alpha=2.5 & 0.31 & 0.30 & (.02) \\ \alpha=1.5 & 0.17 & 0.20 & (.02) \\ \alpha=0.5 & 0.14 & 0.13 & (.02)\end{array}$

\begin{tabular}{cccc}
\multicolumn{3}{c}{ Unmet C/I's } & \\
& & $\begin{array}{c}\text { Posterior } \\
\text { Mean }\end{array}$ & $\begin{array}{c}\text { Posterior } \\
\text { Std. Dev. }\end{array}$ \\
& Actual & 0.13 & $(.01)$ \\
$\mathrm{g}_{1}$ & 0.13 & 0.14 & $(.01)$ \\
$\mathrm{g}_{2}$ & 0.15 & 0.14 & $(.02)$ \\
$\mathrm{g}_{3}$ & 0.22 & 0.24 & $(.01)$ \\
$\mathrm{g}_{4}$ & 0.28 & 0.28 & $(.01)$ \\
$\mathrm{g}_{5}$ & 0.34 & 0.36 & $(.01)$ \\
$\mathrm{g}_{6}$ & 0.55 & 0.54 &
\end{tabular}

\begin{tabular}{|c|c|c|c|c|c|}
\hline \multicolumn{6}{|c|}{ Recovery of Mapping from C/I's to Attributes } \\
\hline \multicolumn{3}{|c|}{ Actual } & \multicolumn{3}{|c|}{ Posterior Means* } \\
\hline$\theta_{\mathrm{jj}}$ & $\theta_{\mathrm{j}, \text { other }}$ & $\theta_{\mathrm{j}, \text { not }}$ & $\theta_{\mathrm{jj}}$ & $\theta_{\mathrm{j}, \mathrm{other}}$ & $\theta_{\mathrm{j}, \mathrm{not}}$ \\
\hline 0.79 & 0.12 & 0.10 & 0.77 & 0.12 & 0.10 \\
\hline 0.74 & 0.10 & 0.16 & 0.75 & 0.08 & 0.17 \\
\hline 0.60 & 0.10 & 0.30 & 0.56 & 0.13 & 0.31 \\
\hline 0.49 & 0.09 & 0.42 & 0.51 & 0.06 & 0.43 \\
\hline 0.37 & 0.11 & 0.52 & 0.33 & 0.13 & 0.53 \\
\hline & & & $\begin{array}{l}\text { All post } \\
\text { osterior }\end{array}$ & $\begin{array}{l}\text { ns are } \\
\text { of actual }\end{array}$ & \\
\hline
\end{tabular}

Table 3.5 Simulation results for the threshold variable selection model, row: one-to-many mapping, multiple observations. 
Simulation results, Column: Many-to-one Mapping, Multiple Observations

\begin{tabular}{|c|c|c|c|c|c|c|c|}
\hline \multicolumn{3}{|c|}{ Distribution of $\beta$ 's } & & \multicolumn{2}{|c|}{ Distribution $\alpha$ 's } & \multirow{3}{*}{$\begin{array}{c}\text { Posterior } \\
\text { Mean }\end{array}$} & \multirow[b]{2}{*}{ Posterior } \\
\hline & Actual & Posterior & Posterior & & & & \\
\hline & Mean & Mean & Std. Dev. & & Actual & & Std. Dev \\
\hline$\beta_{1}$ & 1.90 & 1.93 & $(.06)$ & $\alpha=4.5$ & 0.08 & 0.10 & $(.02)$ \\
\hline$\beta_{2}$ & 1.50 & 1.47 & $(.06)$ & $\alpha=3.5$ & 0.28 & 0.26 & $(.02)$ \\
\hline$\beta_{3}$ & 0.42 & 0.43 & $(.06)$ & $\alpha=2.5$ & 0.30 & 0.30 & $(.02)$ \\
\hline$\beta_{4}$ & -0.94 & -0.48 & $(.06)$ & $\alpha=1.5$ & 0.16 & 0.19 & $(.02)$ \\
\hline$\beta_{5}$ & -0.92 & -0.94 & $(.06)$ & $\alpha=0.5$ & 0.17 & 0.15 & $(.02)$ \\
\hline
\end{tabular}

\begin{tabular}{llcc}
\multicolumn{2}{c}{ Attributes Used } \\
& Actual & $\begin{array}{c}\text { Posterior } \\
\text { Mean }\end{array}$ & $\begin{array}{c}\text { Posterior } \\
\text { Std. Dev. }\end{array}$ \\
$\mathrm{x}_{1}$ & 0.53 & 0.53 & $(.01)$ \\
$\mathrm{x}_{2}$ & 0.45 & 0.45 & $(.01)$ \\
$\mathrm{x}_{3}$ & 0.41 & 0.38 & $(.01)$ \\
$\mathrm{x}_{4}$ & 0.40 & 0.39 & $(.02)$ \\
$\mathrm{x}_{5}$ & 0.30 & 0.29 & $(.01)$
\end{tabular}

\begin{tabular}{llcc}
\multicolumn{3}{c}{ Unmet C/I's } & \\
& Actual & $\begin{array}{c}\text { Posterior } \\
\text { Mean }\end{array}$ & $\begin{array}{c}\text { Posterior } \\
\text { Std. Dev. }\end{array}$ \\
$\mathrm{g}_{1}$ & 0.05 & 0.04 & $(.01)$ \\
$\mathrm{g}_{2}$ & 0.12 & 0.12 & $(.01)$ \\
$\mathrm{g}_{3}$ & 0.17 & 0.21 & $(.02)$ \\
$\mathrm{g}_{4}$ & 0.22 & 0.22 & $(.02)$ \\
$\mathrm{g}_{5}$ & 0.31 & 0.26 & $(.03)$ \\
$\mathrm{g}_{6}$ & 0.54 & 0.53 & $(.01)$
\end{tabular}

Recovery of Mapping from C/I's to Attributes

Actual

$\begin{array}{ccc}\theta_{\mathrm{jj}} & \theta_{\text {other,j }} & \theta_{\text {not,j }} \\ 0.82 & 0.10 & 0.08 \\ 0.69 & 0.11 & 0.20 \\ 0.61 & 0.10 & 0.29 \\ 0.57 & 0.08 & 0.36 \\ 0.41 & 0.90 & 0.50 \\ 0.00 & 0.11 & 0.89\end{array}$

$\begin{array}{ccc}\text { Posterior Means* } & \\ \theta_{\mathrm{jj}} & \theta_{\text {other,j }} & \theta_{\text {not, }} \\ 0.81 & 0.12 & 0.07 \\ 0.68 & 0.12 & 0.20 \\ 0.55 & 0.11 & 0.34 \\ 0.56 & 0.08 & 0.36 \\ 0.39 & 0.18 & 0.43 \\ 0.00 & 0.13 & 0.87\end{array}$

*All posterior means are within $+/-2$

posterior std. dev. of actual values.

Table 3.6 Simulation results for the threshold variable selection model, column: many-toone mapping, multiple observations. 
When only one observation is available with choice and measured c/i's, $\Lambda_{h}$ and $\alpha_{h}$ are not both well identified.

Example using the diagonal, one-to-one mapping.
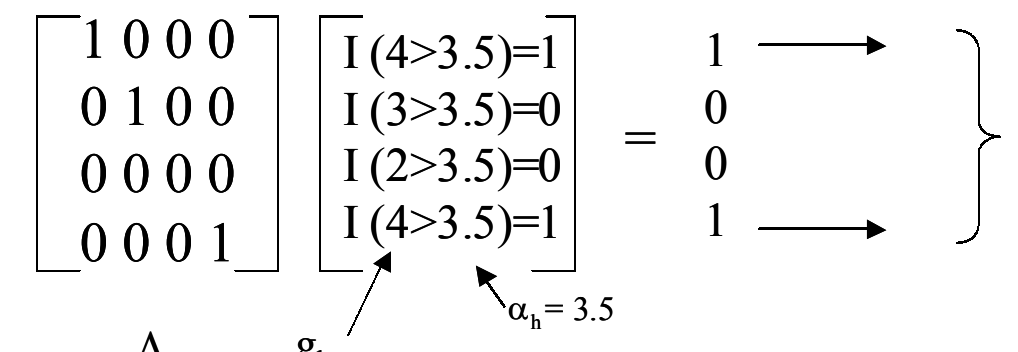

Attributes used to

evaluate brand offerings.

$\Lambda_{\mathrm{h}}$

$\mathrm{g}_{\mathrm{h}}$

$\alpha_{h}=2.5$
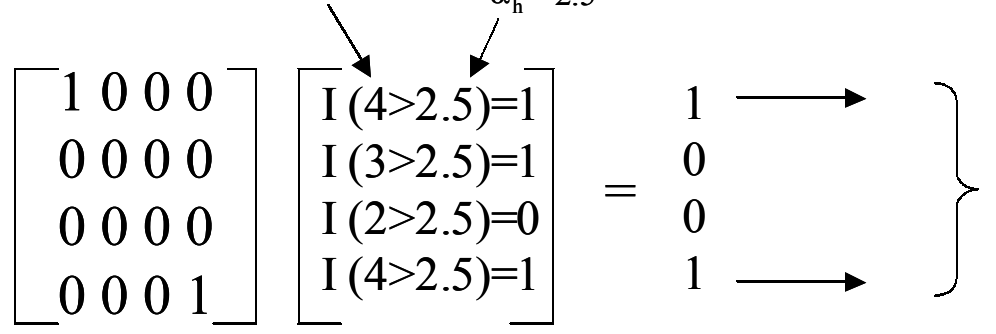

Attributes used to evaluate brand offerings.

Different values of $\Lambda_{\mathrm{h}}$ and $\alpha_{\mathrm{h}}$ yield the same attributes.

Figure 3.5 Example showing that $\Lambda_{\mathrm{h}}$ and $\alpha_{\mathrm{h}}$ are not both well identified with one observed choice. 
Simulation results, Diagonal: One-to-one Mapping, Single Observation - homogeneous scale use

\section{Distribution of $\beta$ 's}

$\begin{array}{lcc} & \begin{array}{c}\text { Actual } \\ \text { Mean }\end{array} & \begin{array}{c}\text { Posterior } \\ \text { Mean }\end{array} \\ \beta_{1} & 2.03 & 2.02 \\ \beta_{2} & 1.45 & 1.44 \\ \beta_{3} & 0.43 & 0.44 \\ \beta_{4} & -0.52 & -0.49 \\ \beta_{5} & -0.95 & -0.96\end{array}$

Attributes Used

$\begin{array}{lccc} & \text { Actual } & \begin{array}{c}\text { Posterior } \\ \text { Mean }\end{array} & \begin{array}{c}\text { Posterior } \\ \text { Std. Dev. }\end{array} \\ \mathrm{x}_{1} & 0.54 & 0.51 & (.02) \\ \mathrm{x}_{2} & 0.49 & 0.55 & (.03) \\ \mathrm{x}_{3} & 0.44 & 0.37 & (.05) \\ \mathrm{x}_{4} & 0.37 & 0.37 & (.04) \\ \mathrm{x}_{5} & 0.31 & 0.36 & (.04)\end{array}$

No heterogeneity in $\alpha$ 's

\begin{tabular}{|llll|}
\hline & Actual & $\begin{array}{c}\text { Posterior } \\
\text { Mean }\end{array}$ & $\begin{array}{c}\text { Posterior } \\
\text { Std. Dev. }\end{array}$ \\
$\alpha=4.5$ & & & \\
$\alpha=3.5$ & 1.00 & & \\
$\alpha=2.5$ & & & \\
$\alpha=1.5$ & & & \\
$\alpha=0.5$ & & & \\
\hline
\end{tabular}

\section{Recovery of Mapping from C/I's to Attributes \\ Actual Posterior Posterior \\ $\theta_{\mathrm{jj}} \quad$ Mean Std. Dev. \\ $0.91 \quad 0.87 \quad(.04)$ \\ $\begin{array}{lll}0.80 & 0.92 \quad(.06)\end{array}$ \\ $\begin{array}{lll}0.75 & 0.60 \quad(.08)\end{array}$ \\ $0.59 \quad 0.62 \quad(.08)$ \\ $\begin{array}{lll}0.54 & 0.59 & (.08)\end{array}$}

Table 3.7 Simulation results for threshold variable selection, diagonal: one-to-one mapping, single observation per respondent, homogeneous scale use. 


\section{Simulation results, Row: One-to-many Mapping, Single Observation - homogeneous scale use}

\section{Distribution of $\beta$ 's}

$\begin{array}{lcc} & \begin{array}{c}\text { Actual } \\ \text { Mean }\end{array} & \begin{array}{c}\text { Posterior } \\ \text { Mean }\end{array} \\ \beta_{1} & 1.99 & 2.01 \\ \beta_{2} & 1.55 & 1.58 \\ \beta_{3} & 0.50 & 0.54 \\ \beta_{4} & -0.52 & -0.51 \\ \beta_{5} & -0.95 & -0.97\end{array}$

\section{Attributes Used}

$\begin{array}{cccc} & \text { Actual } & \begin{array}{c}\text { Posterior } \\ \text { Mean }\end{array} & \begin{array}{c}\text { Posterior } \\ \text { Std. Dev. }\end{array} \\ \mathrm{x}_{1} & 0.54 & 0.52 & (.03) \\ \mathrm{x}_{2} & 0.49 & 0.51 & (.03) \\ \mathrm{x}_{3} & 0.44 & 0.38 & (.06) \\ \mathrm{x}_{4} & 0.35 & 0.41 & (.06) \\ \mathrm{x}_{5} & 0.29 & 0.40 & (.06)\end{array}$

No heterogeneity in $\alpha$ 's

\begin{tabular}{c|cccc|}
\cline { 2 - 4 } $\begin{array}{c}\text { Posterior } \\
\text { Std. Dev. } \\
(.06)\end{array}$ & & & $\begin{array}{c}\text { Posterior } \\
\text { Mean }\end{array}$ & $\begin{array}{c}\text { Posterior } \\
\text { Std. Dev. } \\
(.06)\end{array}$ \\
$(.06)$ & $\alpha=4.5$ & & & \\
$(.06)$ & $\alpha=3.5$ & 1.00 & & \\
$(.06)$ & $\alpha=1.5$ & & & \\
& $\alpha=0.5$ & & & \\
\end{tabular}

\begin{tabular}{cccc}
\multicolumn{3}{c}{ Unmet C/I's } & \\
& & $\begin{array}{c}\text { Posterior } \\
\text { Mean }\end{array}$ & $\begin{array}{c}\text { Posterior } \\
\text { Std. Dev. }\end{array}$ \\
$\mathrm{g}_{1}$ & 0.10 & 0.11 & $(.03)$ \\
$\mathrm{g}_{2}$ & 0.13 & 0.12 & $(.03)$ \\
$\mathrm{g}_{3}$ & 0.21 & 0.28 & $(.05)$ \\
$\mathrm{g}_{4}$ & 0.28 & 0.25 & $(.05)$ \\
$\mathrm{g}_{5}$ & 0.35 & 0.28 & $(.04)$
\end{tabular}

\begin{tabular}{|c|c|c|}
\hline \multicolumn{3}{|c|}{ Recovery of Mapping from C/I's to } \\
\hline Actual & Posterior & Posterio1 \\
\hline$\theta_{\mathrm{jj}}$ & Mean & Std. De \\
\hline 0.81 & 0.74 & $(.06)$ \\
\hline 0.71 & 0.67 & $(.07)$ \\
\hline 0.61 & 0.47 & $(.11)$ \\
\hline 0.48 & 0.43 & $(.11)$ \\
\hline 0.41 & 0.39 & $(.11)$ \\
\hline
\end{tabular}

* Only diagonal elements are shown. Posterior means of all other parameters are also within $+/-2$ posterior std. dev. of actual values.

Table 3.8 Simulation results for threshold variable selection model, row:one-to-many mapping, single observation per respondent, homogeneous scale use. 
Simulation results, Column: Many-to-one Mapping, Single Observation - homogeneous scale use

\section{Distribution of $\beta$ 's}

$\begin{array}{lccc} & \begin{array}{c}\text { Actual } \\ \text { Mean }\end{array} & \begin{array}{c}\text { Posterior } \\ \text { Mean }\end{array} & \begin{array}{c}\text { Posterior } \\ \text { Std. Dev. }\end{array} \\ \beta_{1} & 1.93 & 1.98 & (.08) \\ \beta_{2} & 1.52 & 1.52 & (.07) \\ \beta_{3} & 0.41 & 0.417 & (.06) \\ \beta_{4} & -0.54 & -0.51 & (.05) \\ \beta_{5} & -0.97 & -0.99 & (.06)\end{array}$

\section{Attributes Used}

$\begin{array}{lccc} & \text { Actual } & \begin{array}{c}\text { Posterior } \\ \text { Mean }\end{array} & \begin{array}{c}\text { Posterior } \\ \text { Std. Dev. }\end{array} \\ \mathrm{x}_{1} & 0.50 & 0.47 & (.03) \\ \mathrm{x}_{2} & 0.42 & 0.40 & (.03) \\ \mathrm{x}_{3} & 0.41 & 0.40 & (.05) \\ \mathrm{x}_{4} & 0.30 & 0.27 & (.04) \\ \mathrm{x}_{5} & 0.28 & 0.25 & (.04)\end{array}$

No heterogeneity in $\alpha$ 's

\begin{tabular}{|lccc|}
\hline & Actual & $\begin{array}{c}\text { Posterior } \\
\text { Mean }\end{array}$ & $\begin{array}{c}\text { Posterior } \\
\text { Std. Dev. }\end{array}$ \\
$\alpha=4.5$ & & & \\
$\alpha=3.5$ & 1.00 & & \\
$\alpha=2.5$ & & & \\
$\alpha=1.5$ & & & \\
$\alpha=0.5$ & & & \\
\hline
\end{tabular}

\begin{tabular}{|c|c|c|}
\hline \multicolumn{3}{|c|}{ Recovery of Mapping from C/I's to } \\
\hline Actual & Posterior & Posterior \\
\hline$\theta_{\mathrm{jj}}$ & Mean & Std. Dev. \\
\hline 0.79 & 0.71 & $(.06)$ \\
\hline 0.66 & 0.58 & $(.06)$ \\
\hline 0.59 & 0.57 & $(.11)$ \\
\hline 0.47 & 0.36 & $(.08)$ \\
\hline 0.39 & 0.27 & $(.07)$ \\
\hline
\end{tabular}

\begin{tabular}{cccc}
\multicolumn{3}{c}{ Unmet C/I's } & \\
& & $\begin{array}{c}\text { Posterior } \\
\text { Mean }\end{array}$ & $\begin{array}{c}\text { Posterior } \\
\text { Std. Dev. }\end{array}$ \\
& Actual & 0.09 & $(.04)$ \\
$\mathrm{g}_{1}$ & 0.06 & 0.13 & $(.04)$ \\
$\mathrm{g}_{2}$ & 0.13 & 0.14 & $(.05)$ \\
$\mathrm{g}_{3}$ & 0.17 & 0.24 & $(.06)$ \\
$\mathrm{g}_{4}$ & 0.27 & 0.45 & $(.06)$ \\
$\mathrm{g}_{5}$ & 0.34 & &
\end{tabular}

* Only diagonal elements are shown. Posterior means of all other parameters are also within +/- 2 posterior std. dev. of actual values.

Table 3.9 Simulation results for threshold variable selection model, column: many-to-one mapping, single observation per respondent, homogeneous scale use. 


\section{Simulation results, Diagonal: One-to-one Mapping, Single Observation - heterogeneous scale use}

\section{Distribution of $\beta$ 's}

$\begin{array}{lcc} & \begin{array}{c}\text { Actual } \\ \text { Mean }\end{array} & \begin{array}{c}\text { Posterior } \\ \text { Mean }\end{array} \\ \beta_{1} & 2.03 & 2.06 \\ \beta_{2} & 1.45 & 1.45 \\ \beta_{3} & 0.43 & 0.48 \\ \beta_{4} & -0.52 & -0.51 \\ \beta_{5} & -0.95 & -0.97\end{array}$

Attributes Used

$\begin{array}{ccc} & & \text { Posterio } \\ & \text { Actual } & \text { Mean } \\ \mathrm{x}_{1} & 0.64 & 0.65 \\ \mathrm{x}_{2} & 0.56 & 0.60 \\ \mathrm{x}_{3} & 0.52 & 0.48 \\ \mathrm{x}_{4} & 0.40 & 0.40 \\ \mathrm{x}_{5} & 0.36 & 0.42\end{array}$

Distribution $\alpha$ 's

\begin{tabular}{|cccc|}
\hline & Actual & $\begin{array}{c}\text { Posterior } \\
\text { Mean }\end{array}$ & $\begin{array}{c}\text { Posterior } \\
\text { Std. Dev. }\end{array}$ \\
$\alpha=4.5$ & 0.07 & & \\
$\alpha=3.5$ & 0.39 & Not identified. \\
$\alpha=2.5$ & 0.43 & \\
$\alpha=1.5$ & 0.06 & \\
$\alpha=0.5$ & 0.04 & \\
\hline
\end{tabular}

\section{Recovery of Mapping from C/I's to Attributes}

\begin{tabular}{|ccc|}
\hline Actual & Posterior & Posterior \\
$\theta_{\mathrm{jj}}$ & Mean & Std. Dev. \\
0.91 & & \\
0.78 & Not identified. \\
0.73 & \\
0.57 & \\
0.50 & & \\
\hline
\end{tabular}

Table 3.10 Simulation results for threshold variable selection model, diagonal: one-to-one mapping, single observation per respondent, heterogeneous scale use. 
Simulation results, Row: One-to-many Mapping, Single Observation

- heterogeneous scale use

Distribution of $\beta$ 's

$\begin{array}{lccc} & \begin{array}{c}\text { Actual } \\ \text { Mean }\end{array} & \begin{array}{c}\text { Posterior } \\ \text { Mean }\end{array} & \begin{array}{c}\text { Posterior } \\ \text { Std. Dev. }\end{array} \\ \beta_{1} & 1.99 & 2.03 & (.06) \\ \beta_{2} & 1.55 & 1.57 & (.06) \\ \beta_{3} & 0.50 & 0.55 & (.06) \\ \beta_{4} & -0.52 & -0.52 & (.06) \\ \beta_{5} & -0.95 & -0.97 & (.06)\end{array}$

Attributes Used

Recovery of Mapping from C/I's to Attributes

\begin{tabular}{|ccc|}
\hline Actual & Posterior & Posterior \\
$\theta_{\mathrm{jj}}$ & Mean & Std. Dev. \\
0.80 & & \\
0.70 & Not identified. \\
0.59 & & \\
0.50 & & \\
0.39 & & \\
\hline
\end{tabular}

Distribution $\alpha$ 's

\begin{tabular}{|lccc|}
\hline & Actual & Posterior & Posterior \\
$\alpha=4.5$ & 0.11 & & Std. Dev. \\
$\alpha=3.5$ & 0.37 & Not identified. \\
$\alpha=2.5$ & 0.43 & \\
$\alpha=1.5$ & 0.04 & \\
$\alpha=0.5$ & 0.05 & & \\
\hline
\end{tabular}

\begin{tabular}{|c|c|c|c|c|c|c|c|}
\hline \multicolumn{4}{|c|}{ ttributes Used } & \multicolumn{4}{|c|}{ Unmet C/I's } \\
\hline & & Posterior & Posterior & & & Posterior & Posterior \\
\hline & Actual & Mean & Std. Dev. & & Actual & Mean & Std. Dev. \\
\hline $\mathrm{x}_{1}$ & 0.62 & 0.59 & $(.04)$ & $\mathrm{g}_{1}$ & 0.06 & & \\
\hline $\mathrm{x}_{2}$ & 0.53 & 0.53 & $(.05)$ & $\mathrm{g}_{2}$ & 0.16 & Not ident & \\
\hline $\mathrm{x}_{3}$ & 0.49 & 0.55 & $(.06)$ & $\mathrm{g}_{3}$ & 0.19 & & \\
\hline$x_{4}$ & 0.40 & 0.43 & $(.06)$ & $\mathrm{g}_{4}$ & 0.29 & & \\
\hline $\mathrm{x}_{5}$ & 0.34 & 0.36 & $(.06)$ & $\mathrm{g}_{5}$ & 0.37 & & \\
\hline
\end{tabular}

Table 3.11 Simulation results for the structured variable selection model, row: one-tomany mapping, single observation per respondent, heterogeneous scale use. 


\begin{tabular}{|c|c|c|c|c|c|c|c|}
\hline \multicolumn{4}{|c|}{ Distribution of $\beta$ 's } & \multicolumn{4}{|c|}{ Distribution $\alpha$ 's } \\
\hline & Actual & Posterior & Posterior & & & Posterior & Posterior \\
\hline & Mean & Mean & Std. Dev. & & Actual & Mean & Std. Dev. \\
\hline$\beta_{1}$ & 2.03 & 1.97 & $(.06)$ & $\alpha=4.5$ & 0.11 & & \\
\hline$\beta_{2}$ & 1.42 & 1.37 & $(.06)$ & $\alpha=3.5$ & 0.40 & \multirow{4}{*}{\multicolumn{2}{|c|}{ Not identified. }} \\
\hline$\beta_{3}$ & 0.48 & 0.45 & $(.06)$ & $\alpha=2.5$ & 0.37 & & \\
\hline$\beta_{4}$ & -0.46 & -0.44 & $(.06)$ & $\alpha=1.5$ & 0.06 & & \\
\hline$\beta_{5}$ & -0.96 & -0.87 & $(.07)$ & $\alpha=0.5$ & 0.06 & & \\
\hline
\end{tabular}

\begin{tabular}{|c|c|c|c|c|c|c|c|}
\hline \multicolumn{4}{|c|}{ Attributes Used } & \multicolumn{4}{|c|}{ Unmet C/I's } \\
\hline & & Posterior & Posterior & & & Posterior & Posterior \\
\hline & Actual & Mean & Std. Dev. & & Actual & Mean & Std. Dev. \\
\hline $\mathrm{x}_{1}$ & 0.67 & 0.65 & $(.04)$ & $\mathrm{g}_{1}$ & 0.09 & & \\
\hline $\mathrm{x}_{2}$ & 0.59 & 0.50 & $(.06)$ & $\mathrm{g}_{2}$ & 0.14 & \multirow{4}{*}{\multicolumn{2}{|c|}{ Not identified. }} \\
\hline $\mathrm{x}_{3}$ & 0.49 & 0.43 & $(.06)$ & $\mathrm{g}_{3}$ & 0.20 & & \\
\hline $\mathrm{x}_{4}$ & 0.50 & 0.42 & $(.05)$ & $\mathrm{g}_{4}$ & 0.25 & & \\
\hline $\mathrm{x}_{5}$ & 0.38 & 0.33 & $(.06)$ & $\mathrm{g}_{5}$ & 0.34 & & \\
\hline
\end{tabular}

\section{Recovery of Mapping from C/I's to Attributes}

\begin{tabular}{|ccc|}
\hline Actual & Posterior & Posterior \\
$\theta_{\mathrm{jj}}$ & Mean & Std. Dev. \\
0.86 & & \\
0.80 & Not identified. \\
0.70 & & \\
0.60 & & \\
0.49 & & \\
\hline
\end{tabular}

Table 3.12 Simulation results for the threshold variable selection model, column: manyto-one mapping, single observation per respondent, heterogeneous scale use. 


\section{CHAPTER 4}

\section{EMPIRICAL APPLICATIONS}

This chapter applies the models developed in chapter 3 to two separate data sets. The Medical Device Study has contextual variation in the form of partial and full profile discrete choice conjoint tasks. A total of 16 product attributes are studied. The heterogeneous variable selection, pooled variable selection, and the hybrid models are fit to this data. In the Toothpaste Study, a partial profile rank ordering conjoint task and an actual brand choice are used to infer consumers' preferences. This study examines 30 product attributes and measures 31 concerns and interests that are part of an extended model of choice. In addition to the heterogeneous variable selection and pooled variable selection models, a threshold variable selection model is fit to the toothpaste data.

Both studies support the use of the variable selection models proposed in this thesis. In the Medical Device Study where hold-out predictions are possible, the variable selection models improve both in-sample fit and out-of-sample predictions. The Toothpaste Study shows that concerns and interests add to the explanatory power of the variable selection models. The variable selection models also provide managerial insights beyond the standard models.

The chapter is organized as follows. First the Medical Device Study is presented

followed by the Toothpaste Study. Each section discusses the data, models estimated, the 
results, and managerial insights. Model derivation and estimation algorithms are detailed in chapter 3 and are not repeated with the exception of changes necessary to accommodate the specific data sets.

\subsection{The Medical Device Study}

The heterogeneous variable selection model, pooled variable selection model, and hybrid model are illustrated with a conjoint study on physicians' preferences for a medical device. The study is sponsored by one of the device manufacturers and a professional marketing research firm completed the study design and data collection. Due to the proprietary nature of the data, the product and the majority of product attributes are not revealed. I am grateful to the manufacturer and the marketing research firm for making this data available to me.

The manufacturer identified a total of 16 product attributes for this study. As is common in studies involving a large number of product attributes, respondents completed several tasks in order to ensure that "good" estimates of the attribute weights, or partworths, are obtained. The large number of attributes and the contextual variation make this a good dataset to fit variable selection models. Since explanatory variables that might be used in an extended model of choice are not available, the threshold variable selection model is not fit to this data.

\subsubsection{Data and Models}

Physicians for this study were recruited by the marketing research firm.

Physicians were screened to ensure the medical device under study was pertinent to their 
practice. A total of 111 physicians participated in the study. The questionnaire was administered via personal computers.

Respondents evaluated a total of 19 buying scenarios. Each scenario included 3 products, one each from the three dominant suppliers in the market, including the study's sponsor. Ten of the buying scenarios are "full profile" tasks where each alternative is described on all 16 product attributes including the brand name. Nine of the buying scenarios are "partial profile" tasks where each alternative is described on eight product attributes plus the brand name. The eight product attributes selected for each partial profile task followed an experimental design that ensured the partial profile tasks alone can be used to measure part-worths. In the partial profile task, respondents were instructed to assume that the alternatives were alike on all the omitted product attributes. Half of the respondents saw the partial profile followed by the full profile scenarios; the order was reversed for the other half of respondents. In each buying scenario respondents were asked to indicate which one device they "would be most likely to choose." Thus, the data available for analysis represents multinomial outcomes across two different choice contexts.

Figure 4.1 presents the product attributes, levels, indicator variables, and the orthogonal coding scheme used in the study. The coding scheme results in $28 \beta_{\mathrm{hj}}$ parameters per individual to estimate from the data. Part-worths are obtained by multiplying and adding the appropriate $\beta_{\mathrm{hj}}$ 's across the listed coefficients; the orthogonality constraint makes the part-worths sum to zero across the levels of the attribute. The unique coding scheme facilitates both partial profile analysis and variable selection. When an attribute is not included in a partial profile choice set, the values of 
the indicator variables are set $=0$. Similarly, in the variable selection models, the appropriate $\beta_{\mathrm{hj}}$ 's are set $=0$. By contrast, in standard "dummy variable" coding, for an attribute with 3 levels, both indicator variables are set to 0 to indicate the third level. Thus, standard dummy variable coding cannot be used in this type of analysis.

The models are calibrated with 9 partial profile choice tasks and 8 full profile choice tasks per respondent; 2 full profile choice tasks per respondent are held out for predictive testing. This results in 1,887 observations for calibration and 222 for hold-out validation.

A total of six models are fit to the data. See table 4.1 for a summary. In each model the error term is assumed to be distributed $\operatorname{EV}(0,1)$ resulting in multinomial logit choice probabilities for both the partial profile and full profile choice tasks. Three standard hierarchical multinomial logit models with $\beta_{\mathrm{h}} \sim \operatorname{MVN}\left(\bar{\beta}, \mathrm{V}_{\beta}\right)$ are estimated: one for just the full profile choice tasks, one for just the partial profile choice tasks, and a final model using both the partial and full profile choice tasks. In these models, there is no variable selection and standard methods are used to model heterogeneity.

Three of the proposed variable selection models are also fit to the data. The heterogeneous variable selection model detailed in section 3.1, the pooled variable selection model detailed in section 3.2, and the hybrid model detailed in section 3.3 are estimated. Since the model derivation and estimation algorithms are provided in chapter 3, it will not be repeated here. However, several changes are made consistent with the data. First, as noted above, the likelihood for both sets of observations for each individual is multinomial logit; this results in obvious changes to the estimation algorithms. Second, since one product attribute may be associated with more than one 
indicator variable, variable selection was conducted on pairs of $\beta_{\mathrm{hj}}$ 's for product attributes with three levels. While necessitating more bookkeeping, conceptually the models are the same and the basic structure of the estimation algorithms do not change. Third, in the heterogeneous variable selection (section 3.1.2) and hybrid model (section 3.3.2), in step 1 of both estimation algorithms, the $\beta_{\mathrm{hj}}$ and $\tau_{\mathrm{hj}}$ 's are drawn in blocks corresponding to each attribute. Using the independence chain Metropolis-Hastings algorithm and drawing the complete vectors $\beta_{\mathrm{h}}$ and $\tau_{\mathrm{h}}$ resulted in a relatively slow mixing chain. The only substantive difference to the algorithm is that in step $1, \beta_{h j}^{(n)}$ must now be drawn from a conditional MVN distribution with all the other $\beta_{\mathrm{hj}}$ 's besides $\mathrm{j}$ used as conditioning arguments.

Inference is conducted via Bayesian MCMC methods using the algorithms detailed in chapter 3 with the changes noted above. The chain converged quickly in all models. Convergence was assessed by starting the chains from multiple starting points and inspecting the time series plots of parameters. Each chain was run for 50,000 iterations and a sample of every $10^{\text {th }}$ iteration from the last 25,000 was used to estimate the moments of the posterior distribution.

\subsubsection{Results}

The best fitting model for the in-sample data is the pooled variable selection model while the hybrid model provides the best out-of-sample predictive results. Table 4.1 compares the in-sample and out-of-sample fit for the six models estimated. In-sample fit is assessed by the log marginal density of the data as suggested by Newton and Raftery 
(1994). Out-of-sample fit is measured by the hit rate and the hit probability. Hit rate is the posterior mean of correct predictions for the two hold out choices for each individual, averaged across respondents. Hit probability is the posterior mean of the predicted probability for the selected alternative.

The discrepancy between the best in-sample fit and the best predictive model is due to model averaging. As noted in the literature review, variable selection is a form of model averaging and the log marginal density for any "model" reflects the fit and relative frequency with which the MCMC chain visits each possible combination of variables. The relative frequency is determined by the ratio of the posterior densities in the Metropolis-Hastings algorithm. Unless the "best" model is chosen with probability = 1 on each iteration, the log marginal density will be worse for a variable selection model than for one that included only the best subset of variables. From a theoretical perspective, when there is uncertainty about which model specification is correct, the measure of log marginal density obtained in model averaging represents a more accurate depiction of the uncertainty in the overall model building process.

Model averaging frequently results in better predictive fit than the single "best" fitting model. Madigan and Raftery (1994) provide a theoretical explanation showing that when a logarithmic scoring rule is used, model averaging always results in better predictive ability. Following their argument, let $\Delta$ represent a quantity of interest such as the predicted value of a future observation, the value of a parameter, or the utility of a course of action. Then the posterior distribution of $\Delta$ given the data, $\mathrm{Y}$, is

$$
\operatorname{pr}(\Delta \mid \mathrm{Y})=\sum_{k=1}^{K} \operatorname{pr}\left(\Delta \mid \mathrm{M}_{\mathrm{k}}, \mathrm{Y}\right) \operatorname{pr}\left(\mathrm{M}_{\mathrm{k}} \mid \mathrm{Y}\right)
$$


where $M_{k}$ indexes the set of $K$ models and $\operatorname{pr}\left(M_{k} \mid Y\right)$ is the posterior probability of model $\mathrm{M}_{\mathrm{k}}$ as defined in equations (1) and (2) from chapter 2. The logarithmic scoring rule assigns to each event $A$ that occurs a score of $-\ln \{\operatorname{pr}(\mathrm{A})\}$. Then,

$$
-\mathrm{E}\left[\ln \left\{\sum_{k=1}^{K} \operatorname{pr}\left(\Delta \mid \mathrm{M}_{\mathrm{k}}, \mathrm{Y}\right) \operatorname{pr}\left(\mathrm{M}_{\mathrm{k}} \mid \mathrm{Y}\right)\right\}\right] \leq-\mathrm{E}\left[\ln \left\{\operatorname{pr}\left(\Delta \mid \mathrm{M}_{\mathrm{k}}, \mathrm{Y}\right)\right] \text { for all } \mathrm{K}\right.
$$

where the expectation is taken with respect to $\sum_{k=1}^{K} \operatorname{pr}\left(\Delta \mid \mathrm{M}_{\mathrm{k}}, \mathrm{Y}\right) \operatorname{pr}\left(\mathrm{M}_{\mathrm{k}} \mid \mathrm{Y}\right)$. This result follows from the non-negativity of the Kullback-Leibler information number; see Bickel and Doksum p. 226. Restating (53)

$$
\begin{aligned}
& \mathrm{E}\left[\ln \frac{\sum_{k=1}^{K} \operatorname{pr}\left(\Delta \mid M_{k}, Y\right) \operatorname{pr}\left(M_{k} \mid Y\right)}{\operatorname{pr}\left(\Delta \mid M_{k}, Y\right)}\right] \geq 0 \quad \Rightarrow \\
& \int_{-\infty}^{+\infty}\left[\ln \frac{\sum_{k=1}^{K} \operatorname{pr}\left(\Delta \mid M_{k}, Y\right) \operatorname{pr}\left(M_{k} \mid Y\right)}{\operatorname{pr}\left(\Delta \mid M_{k}, Y\right)}\right]\left[\sum_{k=1}^{K} \operatorname{pr}\left(\Delta \mid M_{k}, Y\right) \operatorname{pr}\left(M_{k} \mid Y\right)\right] d Y \geq 0
\end{aligned}
$$

Note that this result does not extend to the log marginal density of the data since the expectation in (54) integrates over all possible sets of data.

Several studies have documented the superior predictive fit of model averaging. For simulation based and empirical results using both linear and logarithmic scoring rules see Madigan and Raftery (1994), Raftery et al. (1997), and Hoetling et al. (1999). For an example in the marketing literature see Yang and Allenby (2000). It is also interesting to note that in the current data, the "Full profile only" and "Partial profile only" models fit 
their respective portions of the data the best, but result in the worst predictive performance.

Based on the results in table 4.1, the pooled variable selection model fits the insample data better than the baseline model and each of the variable selection models provide better out of sample predictive results. The variable selection models result in a 7 to $16 \%$ improvement in predictive accuracy over the base model. The pooled variable selection model represents a more promising sub-class of models for capturing the data generating process than either the heterogeneous variable selection model or the hybrid model. However, if management is solely interested in prediction, the results favor using the hybrid model.

Table 4.2 compares the posterior means of $\bar{\beta}$ estimated from the baseline model and the three variable selection models. Overwhelmingly, the estimates from the variable selection models have larger absolute values than the estimates from the baseline model. The baseline model differentially shrinks all the estimates towards 0 . The general magnitude of the parameter estimates between the three variable selection models are in agreement. Where discrepancies occur, the heterogeneous and hybrid models tend to have larger posterior means, but also larger posterior standard deviations of the estimates. This typically occurs where less than $1 / 3$ of the respondents are using a given attribute. Recall that the posterior mean in the variable selection models is conditioned on those respondents who actually consider the attribute in their evaluation of the product. With only 111 total respondents, the posterior standard deviation of the estimate increases when relatively few respondents use a given attribute. 
Table 4.3 and 4.4 compare the unexplained heterogeneity in the pooled variable selection and the baseline models, e.g. $\mathrm{V}_{\beta} .25$ of the 28 diagonal elements in the baseline model are smaller in magnitude than the corresponding elements in the pooled variable selection model. Similarly, the pooled variable selection model has a greater number of off-diagonal elements significantly different from 0 . Because the baseline model shrinks the part-worth estimates towards 0 , it understates the amount of heterogeneity in the sample.

Table 4.4 compares the attributes selected across the three variable selection models. The heterogeneous variable selection model suggests that on average, physicians use $37 \%$ or approximately 6 attributes across both the partial and full profile choice tasks. The pooled variable selection model assumes physicians are using all 9 attributes in the partial profile choices, but when faced with the full profile of 16 attributes, select only $45.5 \%$ or 7.3 attributes on average on which to base their decision. The hybrid model indicates that respondents use about $48 \%$ of the 16 attributes in the partial profile analysis and then use about half (53.1\%) of those attributes when faced with the full array of attributes.

The three models all indicate that durability, attribute 5, is the most commonly used attribute. This is a comforting result since the durability of this product directly impacts the well being of the patient. The models also consistently indicate that attribute 1 - brand, and attributes 7 and 8 are among the top 4 attributes used. With the exception of durability, this insight into the market is not obvious from an inspection of just the estimates of $\bar{\beta}$ from the baseline model. 
Results from a market simulation highlight the differences in the models. For each physician in the study, a sample of 500 observations from the posterior distribution of $\beta_{\mathrm{h}}$ for the baseline model and $\beta_{\mathrm{h}}$ and $\lambda_{\mathrm{h}}$ for the pooled variable selection model was drawn. Recall that for the pooled variable selection model, $\lambda_{\mathrm{h}}$ is a vector indicating which attributes are used by individual $\mathrm{h}$ in full profile choice tasks. The simulation is designed to measure the sensitivity in choice probability for attribute 1, brand, and attribute 16 . In the pooled model, about $61 \%$ of physicians use the brand attribute while only $29 \%$ use attribute 16 . Figure 4.2 displays the posterior average difference in choice probability between selected levels of the attributes across the models. For brand, importance is measured as the difference between the choice probability of brand 2 and brand 3, holding all other attributes constant. Brand 2 and brand 3 were selected because they represented the highest and lowest predicted market share. For attribute 16, importance is the difference between the "high" and "low" level of the attribute, averaged across brands, and holding the other attributes fixed.

Figure 4.2 shows that the baseline model does not yield the same measures of attribute importance "on average" as the pooled variable selection model. It might be argued that individual level variable selection is unimportant if ignoring it yields the same market level predictions; figure 4.2 shows this is not the case. The baseline model indicates that the posterior average difference in choice probability between brand 2 and brand 3 is $15.3 \%$. Averaging over physicians who use and don't use brand in their product choice, the pooled variable selection model indicates the average difference in choice probability is $12.8 \%$. For attribute 16 , the baseline model overstates the average change in choice probability by almost $2 \mathrm{x}$ compared to the pooled variable selection 
model. These results indicate that optimizing product configuration using the baseline model will result in a different configuration than the pooled variable selection model. The superior in-sample fit and predictive accuracy of the pooled variable selection model argues for its use over the baseline model.

Figure 4.2 also highlights opportunities for target marketing. Focusing on the results of the pooled variable selection model, the average importance of attribute 16 is relatively low. However, for the $29 \%$ of the market that uses attribute 16 (e.g $\lambda=1$ ), moving from the "low" to the "high" level results in an average change in choice probability of $12.7 \%$. Variable selection models provide a mechanism for identifying the "extremes" of the distribution of heterogeneity and focusing managerial attention on those segments as suggested by Allenby and Ginter (1995).

The empirical results support the variable selection models both from an insample fit and out-of-sample predictive stand-point. In this data, ignoring the issue of variable selection results in biased estimates of the $\bar{\beta}$ 's and underestimating the amount of heterogeneity in the market. Different managerial insights into the market are available by examining which attributes are actually being used by physicians as opposed to just looking at the posterior means of the $\bar{\beta}$ 's in the base model.

In the next section, explanatory variables from an extended model of choice are incorporated into the threshold variable selection model and applied to consumers' preferences for toothpaste. 


\subsection{The Toothpaste Study}

The second empirical study concerns consumers' preferences for brands of toothpaste. The study was designed by Professor Greg Allenby and Dr. Geraldine Fennell and is described in detail in Fennell and Allenby (2003) and Edwards (2002). The original study was designed to test various aspects of Dr. Fennell's model of brand choice, an extended model of choice described in detail in chapter 2. I am grateful to Professor Allenby and Dr. Fennell for making this data available to me.

The threshold variable selection model incorporates explanatory variables and a structure for determining which variables an individual selects. The Toothpaste Study includes measures of concerns and interests and these serve as the explanatory variables. In addition to the threshold variable selection model, the heterogeneous variable selection model and the pooled variable selection model are also estimated. The next section describes the data and the models followed by the results.

\subsubsection{Data and Models}

A nationally representative survey of 863 respondents provided information on concerns and interests related to teeth brushing, desired product attributes, brand evaluations, brand choice, and general oral hygiene questions. Professor Allenby and Dr. Fennell designed the paper and pencil mail survey and it was administered by Yankelovich Partners using its propriety panel of consumers. The variable selection models makes use of four parts of the survey: measures of concerns and interests related to teeth brushing, a partial profile conjoint exercise involving product attributes, consumer's evaluations of the product attributes for each brand, and actual brand choice 
on the last teeth brushing occasion. The likelihood for each respondent consists of the rank ordered conjoint and the actual brand choice.

Thirty-one concerns and interests (c/i's) related to teeth brushing were generated using qualitative research and followed the seven motivational classes suggested by Fennell. Each respondent provided a rating using a 5 point Likert scale on how well each of the c/i's described themselves with anchors "Not at all" and "Completely." A list of all $31 \mathrm{c} / \mathrm{I}$ 's is contained in table 4.10 . These measures provide the relative importance of the $\mathrm{c} / \mathrm{i}^{\mathrm{s}}$, the $\mathrm{g}_{\mathrm{hp}}$ variables used to estimate the threshold variable selection model as discussed in section 3.4.2 .

Thirty product attributes were written to reflect a one-to-one mapping between $\mathrm{c} / \mathrm{i}$ 's and attributes/benefits. The full list of attributes/benefits $(\mathrm{a} / \mathrm{b})$ is provided in table 4.7. An $\mathrm{a} / \mathrm{b}$ for $\mathrm{c} / \mathrm{i} \# 31$, "Toothpastes claim more than they can deliver" was inadvertently excluded from the design. Respondents rated four brands, Aquafresh, Colgate, Crest, and Mentadent on each of the $30 \mathrm{a} / \mathrm{b}$ 's using a 5 point Likert scale anchored by describes "Completely" and "Not at all." Respondents also indicated which, if any, of the four brands was chosen on the last teeth brushing occasion.

A rank ordered partial profile conjoint task was used to elicit the relative importance of the product attributes. Each respondent completed 10 rank order tasks. In each task, four hypothetical products were described using three product attributes. Respondents were instructed to assume that all product attributes not listed were the same across the choices. The partial profile choice tasks were designed to ensure that they alone could be used to measure the relative importance of product attributes. 
The partial profile and actual brand choice data represent choices across two different contexts. In the second task, actual brand choice is modeled as a function of a respondent's ratings on the four brands across the 30 product attributes. For the pooled variable selection model and the threshold variable selection model, only the actual brand choice will be used to infer which product attributes are selected by each respondent. For the heterogeneous variable selection model, variable selection is assumed to occur over both sets of data.

Table 4.6 lists all the models estimated. A baseline model with standard assumptions about heterogeneity and assuming all variables are used in all tasks serves as the null model. The heterogeneous variable selection model is fit to the data following the model derivation and estimation algorithm of sections 3.1.1 and 3.1.2. The only change necessary is recognizing that the likelihood for each respondent consists of two types of data: 10 observations from a rank ordered choice task and a single multinomial brand choice. In both cases an $\operatorname{EV}(0,1)$ error term was assumed and resulted in the rank ordered logit and multinomial logit likelihoods discussed in chapter 3. The pooled variable selection model is fit to the data following the derivation and estimation algorithm of sections 3.2.1 and 3.2.2.

Since only one actual brand choice is available in this data, not all of the parameters in the threshold variable selection model are well identified. Following the discussion in section 3.4.3, two different sets of models are estimated. In the first set, it is assumed that respondents are homogeneous in their use of the 5 point Likert scale used to measure c/i's. A "top two" box assumption is made and a c/i is considered "activated" if it was rated either a "4" or a "5"; this is equivalent to assuming $\alpha_{h}=3.5$ for all households. 
In the second set of models, respondents are assumed to be heterogeneous in their use of the 5 point scale. In this case, $\alpha_{h}$ and $\Lambda_{h}$ are estimated for each individual, but only the product attribute weights and the attributes used are reported.

Consistent with the model derivation in section 3.4.1, diagonal, row restricted, and column restricted maps were estimated for the threshold variable selection model. Recall that the diagonal map assumes a one-to-one correspondence between c/i's and product attributes. This is consistent with the study design. However, Fennell and Allenby (2003) suggest that a complex map exists between c/i's and product attributes. The row restricted map allows a one-to-many relationship between the c/i's and product attributes. The column restricted map allows each product attribute to be responsive to a number of c/i's through a many-to-one mapping. These relationships are summarized in figure 3.5. Three different maps by two different assumptions on scale use result in a total of six threshold variable selection models being fit to the data. Actual estimation followed the algorithms given in section 3.4.2.

Respondents who did not provide complete data on the c/i's or brand attribute ratings were dropped from the data set. A total of 448 respondents provided data on 10 partial profile rank ordered conjoint tasks and one actual brand choice resulting in a total of 4,928 observations to fit the models. Since each respondent provided only one brand choice, no hold-out data is available to assess the predictive accuracy of the models.

Inference is conducted via Bayesian MCMC methods using the algorithms detailed in chapter 3 with the changes noted above. The chain converged quickly in all models. Convergence was assessed by starting the chains from multiple starting points and inspecting the time series plots of parameters. Each chain was run for 50,000 
iterations and a sample of every $10^{\text {th }}$ iteration from the last 15,000 was used to estimate the moments of the posterior distribution.

\subsubsection{Results}

The results indicate that the pooled variable selection model and the various threshold variable selection models fit the data better than the base model or the heterogeneous variable selection model. Model fit is assessed using the log marginal density of the data as suggested by Newton and Raftery (1994) and favors the model with the highest value. Table 4.6 lists the models and the model fit statistics. The better fit of the pooled and threshold models compared to the heterogeneous variable selection model suggests that respondents are giving "good" responses when faced with choice sets involving only limited number of attributes. However, when faced with the full array of product attributes, a regime shift occurs and respondents focus on only a subset of attributes.

Including concerns and interests through the threshold variable selection model improves the explanatory power of the models. In the heterogeneous scale use models, all three maps fit the data better than the pooled variable selection model which does not

include explanatory variables. Overall, the threshold model assuming a column map with a many-to-one mapping between c/i's and product attributes and homogeneous scale use fits the data the best. This is the favored model based on its fit to the data and the fact that it provides estimates of both the attributes used and measures of unmet concerns and interests. 
A comparison of the posterior means of the part-worths for various models is provided in tables 4.7 and 4.8. In general the pooled variable selection model and the favored threshold variable selection model yield posterior estimates of $\overline{\beta_{j}}$ that are greater than the baseline model. However, this occurs in only $2 / 3^{\text {rds }}$ of the cases, indicating that more than a simple scale shift is operating. The models are in broad general agreement on the relative rank of the product attributes, as seen in table 4.8. Six attributes are common in the top 10 across the three models, and seven attributes are common in the bottom 10 across the models. Differences in the absolute values of the parameter estimates are most noticeable in the middle 10 attributes.

Table 4.9 shows the posterior estimate of the unexplained heterogeneity, $\mathrm{V}_{\beta}$, for the favored threshold model. As can be seen, significant heterogeneity exists for attribute 10, "A taste kids love" and attribute 13, "For sensitive teeth."

Table 4.10 provides estimates of the average mapping between the $\mathrm{c} / \mathrm{i}$ 's and the product attributes. Focusing on the first line as an example, for $34 \%$ of the sample, c/i \#1 "I am predisposed to having cavities" maps onto product attribute \#1 "Helps prevent cavities." For $35.2 \%$ of the sample this c/i maps onto some other product attribute and for $28.8 \%$ of the sample it does not map onto any product attribute. The significant mass away from 0 for the off-diagonal elements suggests there is ambiguity in respondents' minds about which product attributes address any one concern and interest. This finding together with the superior fit of the model with the column mapping supports Fennell and Allenby's (2003) earlier findings that a complex relationship exists between c/i's and product attributes. 
Significant heterogeneity exists in the attributes used by consumers when choosing toothpaste. Table 4.11 summarizes the attributes used, sorted by the posterior mean. Recall that in the selection model, "attribute use" is a derived measure based on $\lambda_{\mathrm{hj}}{ }^{\prime}\left(\mathrm{I}\left(\mathrm{g}_{\mathrm{h}}>\alpha\right)\right.$. No attribute is used by more than half of the sample. "Helps you feel good about yourself for brushing regularly" is used most frequently, with $41.1 \%$ of the sample using this product attribute. On average, respondents use 6.4 attributes when choosing a brand of toothpaste.

Table 4.12 lists the unmet concerns and interests as measured by the favored threshold variable selection model. The most frequent unmet c/i is \#31: "Toothpastes claim more than they can deliver." This may be an artifact of this c/i not being explicitly matched to a corresponding product attribute in the study design. Moving beyond the first item, c/i's concerning bad breath, fresh taste, and having a tingle in one's mouth top the list of unmet $\mathrm{c} / \mathrm{i}$ 's. These represent opportunities in the market for manufacturers to reposition their products or introduce new products with attributes that are viewed by consumers as addressing these $\mathrm{c} / \mathrm{i}$ 's.

The threshold variable selection model offers insights beyond those provided by the heterogeneous or pooled variable selection models. Specifically, the map between c/i's and product attributes is estimated and unmet c/i's are identified. Even in a market as mature as toothpaste, opportunities exist to better meet consumers wants and needs. However, in order to identify these opportunities marketing researchers must go "upstream" of current marketplace offerings to the concerns and interests of consumers, as they exist in their everyday life. The threshold variable selection model is a method to 
combine the conceptual models offered by extended models of choice and the statistical models of variable selection.

\subsection{Summary}

This chapter demonstrates the usefulness of the variable selection models across two different data sets. In both data sets variable selection models outperformed the standard model as measured by in-sample fit. The Medical Device Study also shows that the variable selection models provide better out-of-sample predictions. In both data sets, models that allowed a regime shift as respondents moved from one choice context to another fit the data better than the heterogeneous variable selection model. The heterogeneous variable selection model assumes that respondents use the same subset of variables across both choice contexts.

The proposed variable selection models offer greater insights into the market than standard models. In the Medical Device Study, the standard model provided misleading parameter estimates and understated the amount of heterogeneity in the market. In the Toothpaste Study, the threshold variable selection model identified market opportunities that the standard models and other variable selection models could not.

In the final chapter, conclusions and opportunities for extending this stream of research are discussed. 
Medical Device Study, Attributes and Levels

\begin{tabular}{|l|c|l|}
\hline \multicolumn{1}{|c|}{ Attribute } & Number of levels & Indicators \\
\hline Attribute1 - Brand & 3 & $\mathrm{x}_{1}, \mathrm{x}_{2}$ \\
\hline Attribute2 - Price & 3 & $\mathrm{x}_{3}, \mathrm{x}_{4}$ \\
\hline Attribute3 & 3 & $\mathrm{x}_{5,}, \mathrm{x}_{6}$ \\
\hline Attribute4 & 3 & $\mathrm{x}_{7}, \mathrm{x}_{8}$ \\
\hline Attriubte5 - Durability & 3 & $\mathrm{x}_{9}, \mathrm{x}_{10}$ \\
\hline Attrbute6 & 3 & $\mathrm{x}_{11}, \mathrm{x}_{12}$ \\
\hline Attribute7 & 3 & $\mathrm{x}_{13}, \mathrm{x}_{14}$ \\
\hline Attribute8 & 2 & $\mathrm{x}_{15}$ \\
\hline Attribute9 & 3 & $\mathrm{x}_{16}, \mathrm{x}_{17}$ \\
\hline Attribute10 & 2 & $\mathrm{x}_{18}$ \\
\hline Attribute11 & 2 & $\mathrm{x}_{19}$ \\
\hline Attribute12 & 3 & $\mathrm{x}_{20}, \mathrm{x}_{21}$ \\
\hline Attribute13 & 2 & $\mathrm{x}_{22}$ \\
\hline Attribute14 & 3 & $\mathrm{x}_{23}, \mathrm{x}_{24}$ \\
\hline Attribute15 & 3 & $\mathrm{x}_{25}, \mathrm{x}_{26}$ \\
\hline Attriubte16 & 3 & $\mathrm{x}_{27}, \mathrm{x}_{28}$ \\
\hline
\end{tabular}

Example of orthogonal coding: 3 levels

\begin{tabular}{|c|c|c|}
\hline Attribute level & $\mathbf{x}_{\mathbf{j}}$ & $\mathbf{x}_{\mathbf{j}+\mathbf{1}}$ \\
\hline 1 & -.333 & -.333 \\
\hline 2 & .666 & -.333 \\
\hline 3 & -.333 & .666 \\
\hline
\end{tabular}

Example of orthogonal coding: 2 levels

\begin{tabular}{|c|c|}
\hline Attribute level & $\mathbf{x}_{\mathbf{k}}$ \\
\hline 1 & .5 \\
\hline 2 & -.5 \\
\hline
\end{tabular}

Figure 4.1 Attributes, attribute levels, and orthogonal coding for the Medical Device Study. 
Medical Device Variable Selection Model Results

\begin{tabular}{lrrrrr} 
& All & $\begin{array}{c}\text { Full } \\
\text { Profile }\end{array}$ & $\begin{array}{c}\text { Partial } \\
\text { Profile }\end{array}$ & \multicolumn{2}{c}{ Out of Sample } \\
Hit & Hit \\
Model & LMD & LMD & LMD & Rate & Probability \\
Baseline model - all attributes & -890.8 & -379.5 & -550.1 & 0.50 & 0.45 \\
Full profile only & & -337.1 & & 0.46 & 0.42 \\
Partial profile only & & & -478.7 & 0.47 & 0.43 \\
& & & & & \\
Pooled variable selection & -854.8 & -409.4 & -476.3 & 0.54 & 0.48 \\
Heterogeneous variable selection & $-1,118.0$ & -458.2 & -688.3 & 0.56 & 0.49 \\
Hybrid model & $-1,007.2$ & -468.0 & -557.5 & 0.58 & 0.49
\end{tabular}

LMD is log marginal density of the data calculated using the importance sampling method of Newton and Raftery (1994, p. 121)

Table 4.1 Comparison of model fit and predictive results for the Medical Device Study. 
Comparison of $\bar{\beta}$ Estimates: Medical Device Study

\begin{tabular}{|c|c|c|c|c|c|c|c|c|}
\hline \multirow[b]{3}{*}{ Beta } & \multicolumn{2}{|c|}{$\begin{array}{l}\text { Baseline model - } \\
\text { all attributes }\end{array}$} & \multicolumn{2}{|c|}{$\begin{array}{l}\text { Pooled variable } \\
\text { selection model }\end{array}$} & \multicolumn{2}{|c|}{$\begin{array}{l}\text { Heterogeneous } \\
\text { variable selection } \\
\text { model }\end{array}$} & \multicolumn{2}{|c|}{ Hybrid model } \\
\hline & Posterior & Posterior & Posterior & Posterior & Posterior & Posterior & Posterior & Posterior \\
\hline & Mean & Std. Dev. & Mean & Std. Dev. & Mean & Std. Dev. & Mean & Std. Dev. \\
\hline 1 & 0.158 & (.19) & 0.117 & $(.21)$ & 0.236 & $(.28)$ & 0.128 & $(.27)$ \\
\hline 2 & -0.528 & (.19) & -0.733 & $(.23)$ & -0.902 & $(.33)$ & -0.999 & $(.35)$ \\
\hline 3 & 0.248 & $(.22)$ & 0.528 & $(.29)$ & 1.287 & $(.75)$ & 1.884 & $(.89)$ \\
\hline 4 & 0.686 & $(.21)$ & 1.246 & $(.25)$ & 1.872 & $(.76)$ & 2.843 & (.99) \\
\hline 5 & 0.923 & $(.22)$ & 1.225 & (.34) & 2.735 & $(.77)$ & 2.685 & $(.76)$ \\
\hline 6 & 1.329 & $(.22)$ & 1.773 & (.33) & 3.298 & $(.73)$ & 3.369 & $(.75)$ \\
\hline 7 & 0.612 & $(.23)$ & 1.046 & $(.38)$ & 1.024 & $(.51)$ & 1.549 & $(.77)$ \\
\hline 8 & 0.664 & $(.22)$ & 0.956 & (.33) & 1.546 & $(.68)$ & 1.858 & $(.86)$ \\
\hline 9 & 2.288 & $(.27)$ & 2.647 & (.33) & 3.021 & $(.42)$ & 3.211 & (.44) \\
\hline 10 & 2.733 & $(.29)$ & 3.286 & $(.39)$ & 3.872 & (.49) & 4.076 & $(.51)$ \\
\hline 11 & 0.337 & $(.21)$ & 0.456 & $(.31)$ & 1.208 & $(.67)$ & 0.837 & (.79) \\
\hline 12 & 0.561 & $(.21)$ & 0.659 & $(.25)$ & 1.616 & $(.71)$ & 1.357 & $(.73)$ \\
\hline 13 & -0.161 & $(.22)$ & -0.243 & (.24) & -0.313 & $(.35)$ & -0.357 & $(.36)$ \\
\hline 14 & -1.278 & (.23) & -1.674 & $(.37)$ & -2.449 & $(.77)$ & -2.576 & (.66) \\
\hline 15 & 0.458 & $(.21)$ & 0.633 & (.23) & 0.811 & (.37) & 1.001 & (.39) \\
\hline 16 & 0.741 & $(.21)$ & 0.968 & $(.27)$ & 1.496 & (.38) & 1.770 & $(.42)$ \\
\hline 17 & 1.740 & $(.21)$ & 2.229 & $(.28)$ & 2.927 & $(.52)$ & 3.501 & (.53) \\
\hline 18 & 0.061 & (.19) & -0.006 & $(.24)$ & 0.266 & (.61) & 0.061 & (.67) \\
\hline 19 & 0.634 & (.18) & 1.080 & $(.27)$ & 1.135 & $(.55)$ & 1.383 & (.53) \\
\hline 20 & 0.918 & $(.21)$ & 1.365 & $(.29)$ & 2.452 & (1.11) & 2.322 & (.79) \\
\hline 21 & 0.860 & $(.22)$ & 1.334 & $(.26)$ & 2.679 & $(1.13)$ & 2.347 & $(.77)$ \\
\hline 22 & 0.131 & (.19) & 0.113 & (.23) & 1.159 & $(.97)$ & 0.646 & (1.07) \\
\hline 23 & 0.559 & $(.21)$ & 0.426 & $(.26)$ & 1.170 & (.45) & 1.041 & $(.51)$ \\
\hline 24 & 0.920 & $(.21)$ & 1.195 & $(.25)$ & 1.665 & $(.52)$ & 1.874 & (.62) \\
\hline 25 & 0.329 & $(.22)$ & 0.697 & (.29) & 1.375 & (.65) & 2.117 & $(.92)$ \\
\hline 26 & 0.350 & $(.21)$ & 0.728 & (.29) & 1.174 & (.65) & 2.230 & (.97) \\
\hline 27 & 0.186 & $(.23)$ & 0.254 & $(.31)$ & 0.258 & (.43) & 0.040 & (.83) \\
\hline 28 & 0.712 & $(.24)$ & 1.233 & (.34) & 1.403 & $(.51)$ & 2.593 & $(1.01)$ \\
\hline
\end{tabular}

Table 4.2 Comparison of $\bar{\beta}$ estimates for the Medical Device Study. 
The Medical Device Study

Pooled variable selection model

Unobserved heterogeneity - Posterior estimate of covariance matrix $V_{\beta}$

\begin{tabular}{|c|c|c|c|c|c|c|c|c|c|c|}
\hline & 1 & 2 & 3 & 4 & 5 & 6 & 7 & 8 & 9 & 10 \\
\hline 1 & 1.901 & & & & & & & & & \\
\hline 2 & 0.388 & 2.335 & & & & & & & & \\
\hline 3 & -0.068 & -0.419 & 2.530 & & & & & & & \\
\hline 4 & -0.029 & -0.304 & 0.847 & 2.167 & & & & & & \\
\hline 5 & -0.150 & 0.048 & 0.531 & 0.412 & 2.215 & & & & & \\
\hline 6 & 0.006 & 0.010 & 0.506 & 0.379 & 0.807 & 1.899 & & & & \\
\hline 7 & -0.168 & -0.198 & 0.682 & 0.506 & 0.592 & 0.605 & 2.315 & & & \\
\hline 8 & -0.186 & -0.500 & 1.256 & 0.933 & 1.070 & 0.923 & 1.353 & 3.715 & & \\
\hline 9 & -0.244 & -0.317 & 0.726 & 0.508 & 0.819 & 0.641 & 0.864 & 1.561 & 2.687 & \\
\hline 10 & -0.048 & -0.419 & 1.346 & 0.877 & 1.095 & 0.979 & 1.313 & 2.141 & 1.568 & 3.918 \\
\hline 11 & 0.050 & 0.195 & 0.482 & 0.468 & 0.567 & 0.506 & 0.557 & 1.001 & 0.539 & 1.010 \\
\hline 12 & -0.122 & 0.123 & 0.224 & 0.183 & 0.314 & 0.203 & 0.311 & 0.458 & 0.291 & 0.544 \\
\hline 13 & -0.201 & 0.012 & -0.153 & 0.006 & -0.085 & -0.138 & -0.097 & -0.176 & -0.034 & -0.234 \\
\hline 14 & 0.172 & 0.105 & -0.677 & -0.613 & -0.888 & -0.743 & -0.818 & -1.480 & -1.095 & -1.442 \\
\hline 15 & 0.282 & 0.292 & 0.181 & 0.148 & 0.031 & 0.029 & 0.049 & 0.092 & 0.053 & 0.315 \\
\hline 16 & -0.116 & -0.064 & 0.694 & 0.499 & 0.651 & 0.502 & 0.740 & 1.246 & 0.864 & 1.284 \\
\hline 17 & -0.110 & -0.199 & 0.269 & 0.499 & 0.194 & 0.181 & 0.249 & 0.626 & 0.425 & 0.672 \\
\hline 18 & 0.015 & -0.150 & 0.330 & 0.072 & 0.228 & 0.263 & 0.227 & 0.448 & 0.245 & 0.378 \\
\hline 19 & -0.105 & -0.010 & 0.425 & 0.269 & 0.342 & 0.237 & 0.233 & 0.522 & 0.338 & 0.804 \\
\hline 20 & -0.020 & -0.243 & 0.603 & 0.382 & 0.582 & 0.417 & 0.562 & 1.147 & 0.794 & 1.058 \\
\hline 21 & -0.141 & -0.257 & 1.000 & 0.644 & 0.789 & 0.663 & 0.908 & 1.511 & 1.004 & 1.574 \\
\hline 22 & 0.138 & 0.201 & -0.336 & -0.215 & -0.298 & -0.250 & -0.391 & -0.527 & -0.423 & -0.555 \\
\hline 23 & -0.065 & -0.237 & 0.127 & 0.066 & 0.068 & 0.041 & 0.194 & 0.233 & 0.195 & 0.135 \\
\hline 24 & -0.187 & -0.039 & 0.358 & 0.130 & 0.211 & 0.119 & 0.201 & 0.481 & 0.342 & 0.428 \\
\hline 25 & 0.007 & 0.113 & 0.068 & -0.095 & -0.107 & 0.025 & 0.147 & 0.093 & 0.077 & 0.076 \\
\hline 26 & 0.040 & -0.150 & 0.480 & 0.359 & 0.342 & 0.371 & 0.483 & 0.817 & 0.508 & 0.860 \\
\hline 27 & 0.005 & -0.072 & -0.119 & 0.031 & 0.049 & 0.062 & 0.064 & 0.113 & 0.124 & -0.001 \\
\hline 28 & 0.064 & -0.006 & -0.482 & -0.472 & -0.552 & -0.544 & -0.589 & -1.030 & -0.724 & -0.874 \\
\hline
\end{tabular}

Estimates in bold have more than $95 \%$ of posterior mass away from 0 .

Continued

Table 4.3 Posterior estimate of covariance matrix for the Medical Device Study, pooled variable selection model. 
Table 4.3 continued

\begin{tabular}{|c|c|c|c|c|c|c|c|c|c|c|}
\hline & 11 & 12 & 13 & 14 & 15 & 16 & 17 & 18 & 19 & 20 \\
\hline 1 & & & & & & & & & & \\
\hline 2 & & & & & & & & & & \\
\hline 3 & & & & & & & & & & \\
\hline 4 & & & & & & & & & & \\
\hline 5 & & & & & & & & & & \\
\hline 6 & & & & & & & & & & \\
\hline 7 & & & & & & & & & & \\
\hline 8 & & & & & & & & & & \\
\hline 9 & & & & & & & & & & \\
\hline 10 & & & & & & & & & & \\
\hline 11 & 2.114 & & & & & & & & & \\
\hline 12 & 0.412 & 1.835 & & & & & & & & \\
\hline 13 & -0.158 & 0.042 & 1.645 & & & & & & & \\
\hline 14 & -0.830 & -0.286 & 0.240 & 2.707 & & & & & & \\
\hline 15 & 0.246 & 0.098 & -0.164 & -0.080 & 2.036 & & & & & \\
\hline 16 & 0.586 & 0.488 & -0.061 & -0.886 & 0.177 & 2.150 & & & & \\
\hline 17 & 0.310 & 0.306 & -0.113 & -0.620 & 0.162 & 0.623 & 2.707 & & & \\
\hline 18 & 0.184 & 0.050 & -0.098 & -0.321 & -0.100 & 0.174 & -0.024 & 1.691 & & \\
\hline 19 & 0.498 & 0.262 & -0.187 & -0.531 & 0.172 & 0.368 & 0.323 & 0.108 & 1.968 & \\
\hline 20 & 0.533 & 0.391 & -0.014 & -0.903 & 0.058 & 0.709 & 0.455 & 0.367 & 0.252 & 2.390 \\
\hline 21 & 0.762 & 0.160 & -0.107 & -1.086 & 0.170 & 0.791 & 0.243 & 0.397 & 0.550 & 0.876 \\
\hline 22 & -0.164 & -0.043 & 0.061 & 0.435 & -0.026 & -0.268 & -0.123 & -0.152 & -0.143 & -0.361 \\
\hline 23 & -0.051 & -0.117 & 0.006 & 0.042 & -0.004 & 0.070 & -0.201 & 0.069 & -0.169 & -0.078 \\
\hline 24 & 0.228 & 0.142 & -0.212 & -0.251 & 0.175 & 0.341 & 0.281 & -0.008 & 0.389 & 0.203 \\
\hline 25 & 0.102 & 0.006 & 0.031 & -0.097 & -0.048 & 0.017 & 0.062 & 0.040 & 0.044 & 0.179 \\
\hline 26 & 0.382 & 0.323 & -0.020 & -0.459 & 0.080 & 0.512 & 0.372 & 0.235 & 0.213 & 0.482 \\
\hline 27 & 0.075 & 0.068 & -0.086 & -0.051 & -0.031 & -0.011 & -0.014 & -0.047 & 0.024 & -0.050 \\
\hline 28 & -0.567 & -0.029 & 0.177 & 0.909 & -0.062 & -0.509 & -0.565 & -0.072 & -0.187 & -0.386 \\
\hline
\end{tabular}

Estimates in bold have more than $95 \%$ of posterior mass away from 0 .

Continued 
Table 4.3 continued

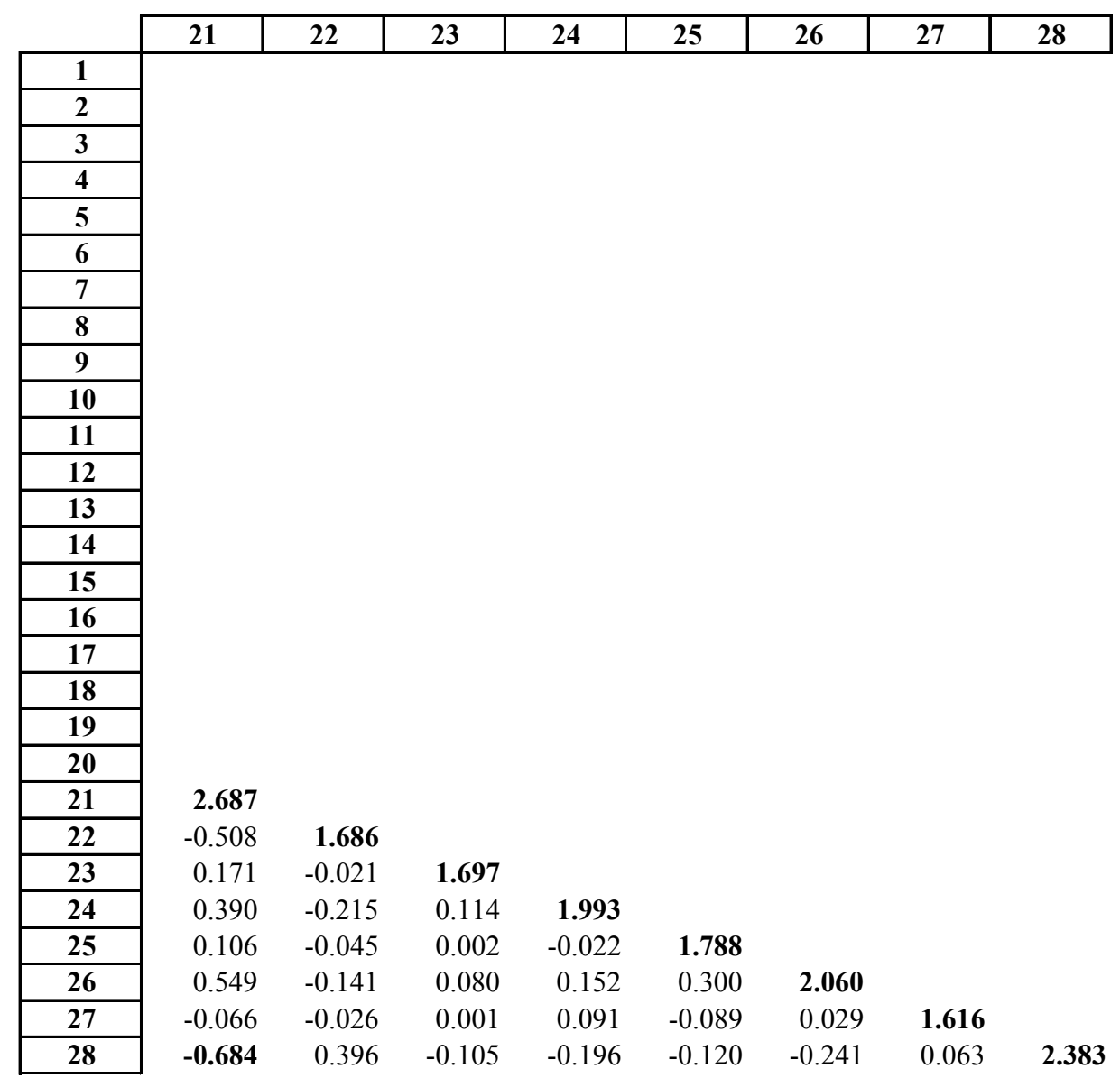

Estimates in bold have more than $95 \%$ of posterior mass away from 0 . 
The Medical Device Study

Baseline model -- all attributes included

Unobserved heterogeneity - Posterior estimate of covariance matrix $V_{\beta}$

\begin{tabular}{|c|c|c|c|c|c|c|c|c|c|c|}
\hline & 1 & 2 & 3 & 4 & 5 & 6 & 7 & 8 & 9 & 10 \\
\hline 1 & 1.937 & & & & & & & & & \\
\hline 2 & 0.436 & 2.112 & & & & & & & & \\
\hline 3 & 0.006 & -0.177 & 2.102 & & & & & & & \\
\hline 4 & -0.005 & -0.224 & $\mathbf{0 . 5 5 7}$ & 1.848 & & & & & & \\
\hline 5 & -0.081 & 0.025 & 0.428 & 0.285 & 1.916 & & & & & \\
\hline 6 & 0.068 & 0.124 & 0.377 & 0.170 & 0.680 & 1.711 & & & & \\
\hline 7 & -0.091 & -0.002 & 0.397 & 0.199 & 0.260 & 0.268 & 1.602 & & & \\
\hline 8 & 0.006 & -0.206 & 0.436 & 0.334 & 0.551 & 0.420 & 0.487 & 2.248 & & \\
\hline 9 & -0.355 & -0.341 & 0.327 & 0.270 & 0.541 & 0.481 & 0.366 & 1.000 & 3.065 & \\
\hline 10 & -0.149 & -0.421 & 0.879 & 0.511 & 0.721 & 0.622 & 0.605 & 1.138 & 1.992 & 4.173 \\
\hline 11 & 0.134 & 0.201 & 0.181 & 0.129 & 0.232 & 0.238 & 0.211 & 0.354 & 0.155 & 0.453 \\
\hline 12 & -0.149 & 0.069 & 0.024 & -0.082 & 0.125 & 0.037 & 0.039 & 0.110 & 0.243 & 0.269 \\
\hline 13 & -0.264 & 0.050 & -0.187 & -0.013 & -0.227 & -0.221 & -0.049 & -0.227 & -0.017 & -0.222 \\
\hline 14 & 0.086 & 0.060 & -0.211 & -0.055 & -0.330 & -0.290 & -0.131 & -0.572 & -0.591 & -0.490 \\
\hline 15 & 0.313 & 0.228 & 0.110 & 0.082 & 0.006 & 0.007 & -0.033 & -0.039 & 0.050 & 0.341 \\
\hline 16 & -0.168 & -0.106 & 0.259 & 0.153 & 0.421 & 0.296 & 0.258 & 0.525 & 0.856 & 1.153 \\
\hline 17 & -0.219 & -0.402 & 0.164 & 0.297 & 0.191 & 0.089 & 0.067 & 0.526 & 0.894 & 0.987 \\
\hline 18 & 0.120 & -0.031 & 0.151 & -0.176 & 0.042 & 0.142 & -0.060 & 0.144 & 0.026 & 0.112 \\
\hline 19 & -0.148 & -0.109 & 0.157 & 0.101 & 0.042 & -0.021 & 0.032 & 0.168 & 0.196 & 0.390 \\
\hline 20 & -0.033 & -0.162 & 0.271 & -0.034 & 0.122 & 0.046 & 0.035 & 0.339 & 0.388 & 0.512 \\
\hline 21 & -0.102 & -0.043 & 0.505 & 0.190 & 0.435 & 0.343 & 0.322 & 0.548 & 0.551 & 0.794 \\
\hline 22 & 0.046 & 0.089 & -0.275 & -0.119 & -0.192 & -0.118 & -0.156 & -0.180 & -0.233 & -0.408 \\
\hline 23 & -0.111 & -0.187 & 0.165 & 0.118 & 0.146 & 0.101 & 0.061 & 0.216 & 0.417 & 0.350 \\
\hline 24 & -0.284 & -0.051 & 0.323 & 0.209 & 0.148 & 0.057 & 0.184 & 0.312 & 0.568 & 0.571 \\
\hline 25 & 0.001 & 0.095 & -0.033 & -0.157 & -0.290 & -0.133 & -0.023 & -0.029 & -0.105 & -0.317 \\
\hline 26 & 0.063 & -0.017 & 0.171 & 0.080 & -0.018 & 0.025 & 0.112 & 0.212 & 0.402 & 0.472 \\
\hline 27 & -0.084 & -0.174 & -0.108 & 0.079 & -0.028 & -0.021 & 0.060 & 0.075 & 0.196 & 0.091 \\
\hline 28 & -0.022 & 0.084 & -0.219 & -0.387 & -0.351 & -0.335 & -0.184 & -0.523 & -0.495 & -0.358 \\
\hline
\end{tabular}

Estimates in bold have more than $95 \%$ of posterior mass away from 0 .

Continued

Table 4.4 Posterior estimate of covariance matrix for the Medical Device Study, baseline model - all attributes included. 
Table 4.4 continued

\begin{tabular}{|c|c|c|c|c|c|c|c|c|c|c|}
\hline & 11 & 12 & 13 & 14 & 15 & 16 & 17 & 18 & 19 & 20 \\
\hline 1 & & & & & & & & & & \\
\hline 2 & & & & & & & & & & \\
\hline 3 & & & & & & & & & & \\
\hline 4 & & & & & & & & & & \\
\hline 5 & & & & & & & & & & \\
\hline 6 & & & & & & & & & & \\
\hline 7 & & & & & & & & & & \\
\hline 8 & & & & & & & & & & \\
\hline 9 & & & & & & & & & & \\
\hline 10 & & & & & & & & & & \\
\hline 11 & 1.589 & & & & & & & & & \\
\hline 12 & 0.253 & 1.510 & & & & & & & & \\
\hline 13 & -0.099 & 0.044 & 1.688 & & & & & & & \\
\hline 14 & -0.331 & -0.022 & 0.262 & 2.151 & & & & & & \\
\hline 15 & 0.067 & -0.097 & -0.134 & 0.026 & 1.942 & & & & & \\
\hline 16 & 0.173 & 0.194 & -0.045 & -0.473 & -0.002 & 1.854 & & & & \\
\hline 17 & 0.139 & 0.200 & -0.115 & -0.443 & 0.080 & 0.669 & 2.625 & & & \\
\hline 18 & 0.158 & -0.074 & -0.163 & -0.277 & -0.118 & 0.031 & 0.007 & 1.559 & & \\
\hline 19 & 0.122 & 0.098 & -0.251 & -0.195 & 0.018 & 0.163 & 0.340 & 0.136 & 1.534 & \\
\hline 20 & 0.221 & 0.107 & 0.035 & -0.570 & -0.059 & 0.317 & 0.301 & 0.344 & 0.180 & 1.891 \\
\hline 21 & 0.317 & -0.128 & -0.117 & -0.579 & 0.058 & 0.420 & 0.112 & 0.319 & 0.185 & 0.563 \\
\hline 22 & -0.022 & 0.016 & 0.074 & 0.071 & -0.058 & -0.054 & 0.063 & -0.041 & -0.046 & -0.144 \\
\hline 23 & -0.084 & -0.048 & -0.001 & -0.037 & 0.087 & 0.184 & 0.138 & 0.005 & 0.084 & 0.036 \\
\hline 24 & 0.035 & 0.071 & -0.193 & -0.084 & 0.243 & 0.254 & 0.413 & -0.186 & 0.277 & 0.021 \\
\hline 25 & -0.013 & 0.005 & 0.046 & -0.016 & -0.168 & -0.093 & -0.070 & 0.122 & 0.069 & 0.091 \\
\hline 26 & 0.077 & 0.132 & 0.062 & -0.059 & 0.003 & 0.154 & 0.377 & 0.084 & -0.004 & 0.091 \\
\hline 27 & -0.029 & 0.115 & 0.065 & 0.098 & -0.076 & 0.048 & 0.074 & -0.178 & -0.014 & -0.120 \\
\hline 28 & -0.155 & 0.114 & 0.274 & 0.536 & -0.099 & -0.235 & -0.361 & 0.018 & -0.056 & -0.068 \\
\hline
\end{tabular}

Estimates in bold have more than $95 \%$ of posterior mass away from 0 .

Continued 
Table 4.4 continued

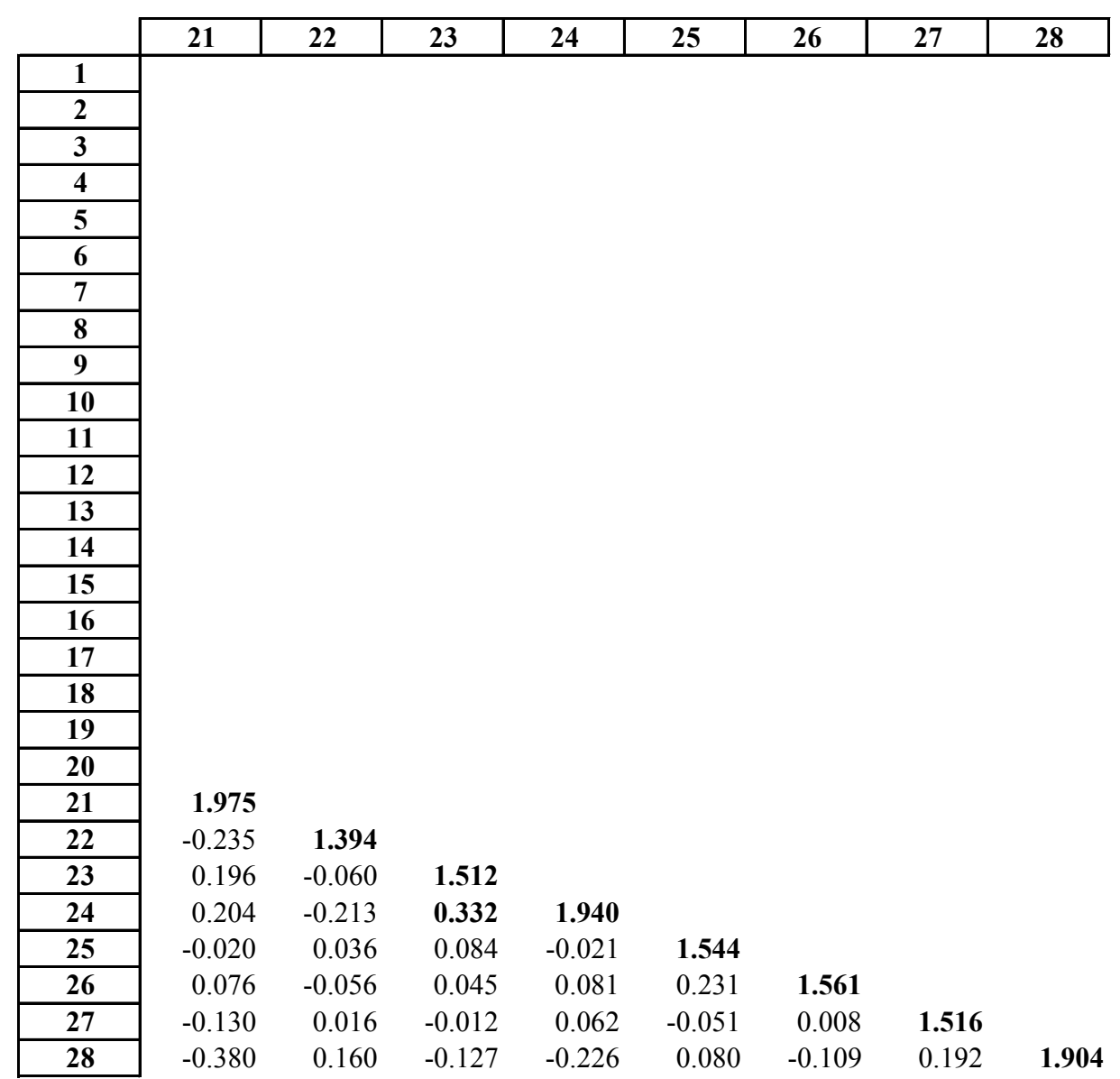

Estimates in bold have more than $95 \%$ of posterior mass away from 0 . 


\section{Comparison of Attributes Selected: Medical Device Study}

\begin{tabular}{|c|c|c|c|c|}
\hline \multirow{4}{*}{$\begin{array}{c}\text { Attribute } \\
1\end{array}$} & \multicolumn{2}{|c|}{$\begin{array}{l}\text { Heterogeneous } \\
\text { variable selection model } \\
\text { estimates of } \theta\end{array}$} & \multicolumn{2}{|c|}{$\begin{array}{l}\text { Pooled variable } \\
\text { selection model } \\
\text { estimates of } \theta\end{array}$} \\
\hline & Posterior & Posterior & Posterior & Posterior \\
\hline & Mean & Std. Dev. & Mean & Std. Dev. \\
\hline & 0.477 & (.09) & 0.611 & $(.11)$ \\
\hline 2 & 0.363 & (.13) & 0.430 & (.11) \\
\hline 3 & 0.312 & (.08) & 0.550 & (.11) \\
\hline 4 & 0.370 & (.12) & 0.407 & $(.12)$ \\
\hline 5 & 0.564 & $(.07)$ & 0.647 & $(.07)$ \\
\hline 6 & 0.226 & $(.09)$ & 0.410 & $(.12)$ \\
\hline 7 & 0.435 & $(.12)$ & 0.625 & $(.11)$ \\
\hline 8 & 0.485 & (.14) & 0.516 & $(.15)$ \\
\hline 9 & 0.468 & $(.09)$ & 0.575 & $(.09)$ \\
\hline 10 & 0.320 & (.14) & 0.379 & $(.15)$ \\
\hline 11 & 0.388 & $(.14)$ & 0.337 & (.13) \\
\hline 12 & 0.313 & (.12) & 0.463 & (.11) \\
\hline 13 & 0.212 & $(.11)$ & 0.355 & $(.15)$ \\
\hline 14 & 0.400 & (.11) & 0.433 & $(.12)$ \\
\hline 15 & 0.240 & (.09) & 0.243 & $(.11)$ \\
\hline 16 & 0.354 & $(.12)$ & 0.292 & (.09) \\
\hline
\end{tabular}

\begin{tabular}{cccccc|}
\cline { 2 - 5 } & \multicolumn{4}{c|}{ Hybrid model } \\
& estimates of $\phi$ & \multicolumn{2}{c|}{ Posterior } & Posterior \\
\cline { 2 - 6 } Attribute & Mesterior & Posterior & Std. Dev. & Mean & Std. Dev. \\
$\mathbf{1}$ & 0.605 & $(.12)$ & 0.696 & $(.11)$ \\
$\mathbf{2}$ & 0.422 & $(.14)$ & 0.447 & $(.14)$ \\
$\mathbf{3}$ & 0.409 & $(.11)$ & 0.643 & $(.12)$ \\
$\mathbf{4}$ & 0.455 & $(.15)$ & 0.590 & $(.15)$ \\
$\mathbf{5}$ & 0.653 & $(.07)$ & 0.739 & $(.08)$ \\
$\mathbf{6}$ & 0.430 & $(.17)$ & 0.495 & $(.15)$ \\
$\mathbf{7}$ & 0.520 & $(.13)$ & 0.687 & $(.11)$ \\
$\mathbf{8}$ & 0.574 & $(.15)$ & 0.579 & $(.15)$ \\
$\mathbf{9}$ & 0.563 & $(.09)$ & 0.604 & $(.09)$ \\
$\mathbf{1 0}$ & 0.387 & $(.18)$ & 0.480 & $(.18)$ \\
$\mathbf{1 1}$ & 0.553 & $(.16)$ & 0.453 & $(.15)$ \\
$\mathbf{1 2}$ & 0.506 & $(.15)$ & 0.444 & $(.13)$ \\
$\mathbf{1 3}$ & 0.323 & $(.17)$ & 0.462 & $(.18)$ \\
$\mathbf{1 4}$ & 0.510 & $(.14)$ & 0.540 & $(.14)$ \\
$\mathbf{1 5}$ & 0.351 & $(.12)$ & 0.348 & $(.15)$ \\
$\mathbf{1 6}$ & 0.413 & $(.15)$ & 0.289 & $(.11)$
\end{tabular}

Table 4.5 Comparison of attributes selected: Medical Device Study 
Medical Device Study

Comparison of Attribute Importance

Baseline and Pooled Variable Selection Models

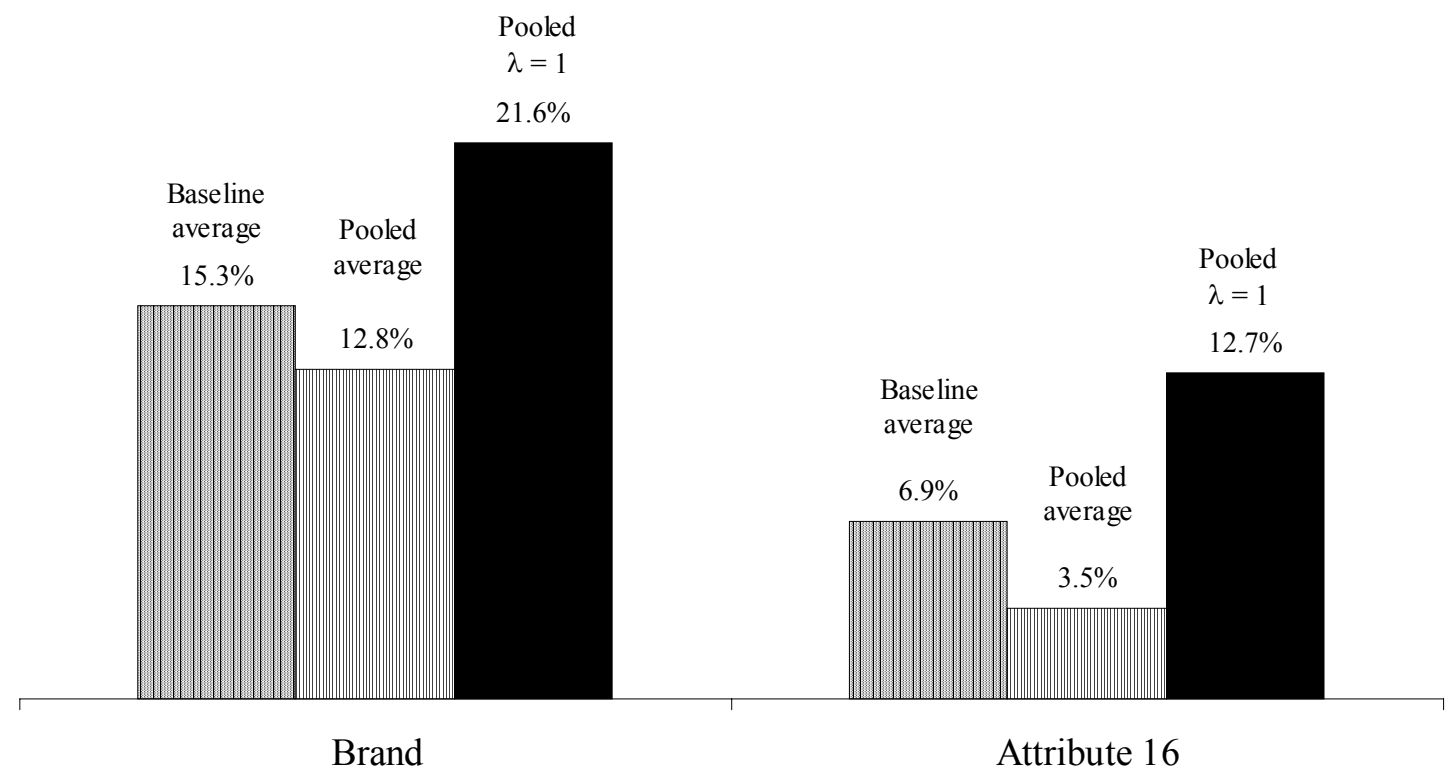

Figure 4.2 Comparison of selected attribute importance: Medical Device Study. 


\title{
Toothpast Study: Model Fit Comparison
}

\author{
Model $\underline{\text { LMD }}$ \\ Baseline model -- all variables $\quad-10,195.8$ \\ Heterogeneous variable selection $\quad-10,549.7$ \\ Pooled variable selection $\quad-10,075.3$ \\ Threshold models - with homogeneous scale use \\ Diagonal: one-to-one mapping $\quad-10,139.0$ \\ Row: one-to-many mapping $\quad-10,086.9$ \\ Column: many-to-one mapping $\quad-9,988.6$ \\ Threshold models - with heterogeneous scale use \\ $\begin{array}{lr}\text { Diagonal: one-to-one mapping } & -10,051.6 \\ \text { Row: one-to-many mapping } & -9,991.0 \\ \text { Column: many-to-one mapping } & -9,989.0\end{array}$
}

LMD is log marginal density of the data calculated using the importance sampling method of Newton and Raftery (1994, p. 121)

Table 4.6 Comparison of model fit for the Toothpaste Study. 


\begin{tabular}{|c|c|c|c|c|c|c|c|}
\hline \multirow[b]{2}{*}{ Attribute/benefit } & \multirow[b]{2}{*}{ Beta } & \multicolumn{2}{|c|}{ Baseline model - all variables } & \multicolumn{2}{|c|}{$\begin{array}{l}\text { Pooled variable } \\
\text { selection model }\end{array}$} & \multicolumn{2}{|c|}{$\begin{array}{l}\text { Threshold model } \\
\text { - with homo. scale use } \\
\text { Column: many-to-one mapping }\end{array}$} \\
\hline & & $\begin{array}{l}\text { Posterior } \\
\text { Mean }\end{array}$ & $\begin{array}{l}\text { Posterior } \\
\text { Std. Dev. }\end{array}$ & $\begin{array}{l}\text { Posterior } \\
\text { Mean }\end{array}$ & $\begin{array}{l}\text { Posterior } \\
\text { Std. Dev. }\end{array}$ & $\begin{array}{c}\text { Posterior } \\
\text { Mean }\end{array}$ & $\begin{array}{l}\text { Posterior } \\
\text { Std. Dev. }\end{array}$ \\
\hline Helps prevent cavities & 1 & 0.607 & $(.13)$ & 1.847 & $(.41)$ & 1.598 & $(.26)$ \\
\hline Delivers protection in hard to reach places & 2 & 0.254 & $(.14)$ & 1.490 & $(.43)$ & 1.216 & $(.27)$ \\
\hline Helps remove tartar \& plaque & 3 & 0.831 & $(.15)$ & 2.196 & $(.42)$ & 2.016 & $(.23)$ \\
\hline Helps promote healthy gums & 4 & 0.356 & $(.11)$ & 1.589 & $(.41)$ & 1.387 & $(.24)$ \\
\hline Penetrates to strengthen your teeth against & 5 & 0.902 & $(.13)$ & 2.132 & (.44) & 1.918 & $(.23)$ \\
\hline Helps fight germs \& infections in your mouth & 6 & 0.190 & $(.14)$ & 1.420 & $(.43)$ & 1.220 & $(.24)$ \\
\hline Mild tasting & 7 & 0.452 & $(.12)$ & 1.111 & $(.19)$ & 1.018 & $(.24)$ \\
\hline Fresh tasting & 8 & 1.417 & $(.11)$ & 2.137 & $(.19)$ & 2.016 & $(.25)$ \\
\hline Gives your mouth a tingle & 9 & 0.682 & $(.12)$ & 1.385 & $(.21)$ & 1.319 & $(.24)$ \\
\hline \begin{tabular}{|l|} 
A taste kid's love \\
\end{tabular} & 10 & -0.464 & $(.14)$ & 0.099 & $(.22)$ & 0.056 & $(.28)$ \\
\hline Great bubbling action & 11 & -0.229 & $(.13)$ & 0.490 & $(.21)$ & 0.443 & $(.32)$ \\
\hline \begin{tabular}{|l|} 
Doesn't irritate your mouth \\
\end{tabular} & 12 & 0.791 & $(.19)$ & 0.859 & $(.26)$ & 2.265 & $(.26)$ \\
\hline For sensitive teeth & 13 & 0.326 & $(.17)$ & 0.333 & $(.22)$ & 1.793 & $(.23)$ \\
\hline Safe for tooth enamel (non-scratching) & 14 & 0.912 & $(.19)$ & 0.874 & $(.23)$ & 2.294 & $(.26)$ \\
\hline Helps clean teeth. & 15 & 0.409 & $(.15)$ & 0.542 & $(.15)$ & 0.269 & $(.13)$ \\
\hline Helps remove stains & 16 & 0.245 & $(.13)$ & 0.401 & $(.16)$ & 0.203 & $(.18)$ \\
\hline \begin{tabular}{|l|} 
Whitens your teeth \\
\end{tabular} & 17 & 0.394 & $(.12)$ & 0.513 & $(.15)$ & 0.302 & $(.13)$ \\
\hline Makes your teeth gleam like pearls & 18 & -0.639 & $(.13)$ & -0.521 & $(.15)$ & -0.682 & $(.16)$ \\
\hline Fights bad breath & 19 & -0.567 & $(.21)$ & 0.051 & $(.18)$ & -0.290 & $(.21)$ \\
\hline Freshens breath for 12 hours & 20 & -0.336 & $(.24)$ & 0.299 & $(.18)$ & -0.076 & $(.24)$ \\
\hline Helps take away morning breath & 21 & -0.513 & $(.22)$ & 0.131 & $(.22)$ & -0.258 & $(.23)$ \\
\hline $20 \%$ less than regular price & 22 & 0.875 & $(.11)$ & 0.893 & $(.09)$ & 0.844 & $(.09)$ \\
\hline $100 \%$ natural ingredients & 23 & 0.746 & $(.11)$ & 0.753 & $(.11)$ & 0.764 & $(.11)$ \\
\hline $100 \%$ recyclable packaging & 24 & 0.506 & $(.09)$ & 0.509 & $(.08)$ & 0.449 & $(.11)$ \\
\hline For routine maintenance & 25 & 2.202 & $(.24)$ & 1.914 & $(.34)$ & 2.808 & $(.22)$ \\
\hline Provides a change of pace & 26 & -1.315 & $(.13)$ & -2.223 & $(.35)$ & -1.087 & $(.19)$ \\
\hline Helps you feel good about yourself for & 27 & 1.952 & $(.16)$ & 2.786 & $(.34)$ & 2.080 & $(.29)$ \\
\hline An interesting way to clean teeth & 28 & -1.091 & $(.12)$ & -1.968 & $(.34)$ & -0.854 & $(.16)$ \\
\hline Shows others you care about your teeth & 29 & 0.654 & $(.16)$ & 1.484 & $(.35)$ & 0.835 & $(.28)$ \\
\hline For everyday brushing & 30 & 2.217 & $(.25)$ & 1.927 & $(.36)$ & 2.868 & $(.22)$ \\
\hline
\end{tabular}

Table 4.7 Comparison of part-worth estimates for the Toothpaste Study. 
Comparison of Poseterior Means of Part-worth estimates - Sorted by relative size

\begin{tabular}{|c|c|c|c|c|c|c|c|c|}
\hline & \multicolumn{2}{|c|}{$\begin{array}{l}\text { Baseline model } \\
\text { - all variables }\end{array}$} & \multicolumn{3}{|c|}{$\begin{array}{l}\text { Pooled variable } \\
\text { selection model }\end{array}$} & \multicolumn{3}{|c|}{$\begin{array}{l}\text { - with homo. scale use } \\
\text { Column: many-to-one mapping }\end{array}$} \\
\hline & Posterior & Posterior & & Posterior & Posterior & & Posterior & Posterior \\
\hline Beta & Mean & Std. Dev. & Beta & Mean & Std. Dev. & Beta & Mean & Std. Dev. \\
\hline 30 & 2.217 & $(.25)$ & 27 & 2.786 & $(.34)$ & 30 & 2.868 & $(.22)$ \\
\hline 25 & 2.202 & $(.24)$ & 3 & 2.196 & $(.42)$ & 25 & 2.808 & $(.22)$ \\
\hline 27 & 1.952 & $(.16)$ & 8 & 2.137 & (.19) & 14 & 2.294 & $(.26)$ \\
\hline 8 & 1.417 & $(.11)$ & 5 & 2.132 & (.44) & 12 & 2.265 & $(.26)$ \\
\hline 14 & 0.912 & (.19) & 30 & 1.927 & $(.36)$ & 27 & 2.080 & $(.29)$ \\
\hline 5 & 0.902 & (.13) & 25 & 1.914 & $(.34)$ & 3 & 2.016 & $(.23)$ \\
\hline 22 & 0.875 & $(.11)$ & 1 & 1.847 & $(.41)$ & 8 & 2.016 & $(.25)$ \\
\hline 3 & 0.831 & $(.15)$ & 4 & 1.589 & (.41) & 5 & 1.918 & $(.23)$ \\
\hline 12 & 0.791 & (.19) & 2 & 1.490 & (.43) & 13 & 1.793 & $(.23)$ \\
\hline 23 & 0.746 & (.11) & 29 & 1.484 & (.35) & 1 & 1.598 & $(.26)$ \\
\hline 9 & 0.682 & (.12) & 6 & 1.420 & (.43) & 4 & 1.387 & $(.24)$ \\
\hline 29 & 0.654 & (.16) & 9 & 1.385 & $(.21)$ & 9 & 1.319 & $(.24)$ \\
\hline 1 & 0.607 & (.13) & 7 & 1.111 & (.19) & 6 & 1.220 & $(.24)$ \\
\hline 24 & 0.506 & (.09) & 22 & 0.893 & (.09) & 2 & 1.216 & $(.27)$ \\
\hline 7 & 0.452 & (.12) & 14 & 0.874 & (.23) & 7 & 1.018 & $(.24)$ \\
\hline 15 & 0.409 & (.15) & 12 & 0.859 & $(.26)$ & 22 & 0.844 & $(.09)$ \\
\hline 17 & 0.394 & (.12) & 23 & 0.753 & (.11) & 29 & 0.835 & $(.28)$ \\
\hline 4 & 0.356 & (.11) & 15 & 0.542 & (.15) & 23 & 0.764 & $(.11)$ \\
\hline 13 & 0.326 & (.17) & 17 & 0.513 & (.15) & 24 & 0.449 & $(.11)$ \\
\hline 2 & 0.254 & (.14) & 24 & 0.509 & (.08) & 11 & 0.443 & $(.32)$ \\
\hline 16 & 0.245 & (.13) & 11 & 0.490 & $(.21)$ & 17 & 0.302 & $(.13)$ \\
\hline 6 & 0.190 & (.14) & 16 & 0.401 & (.16) & 15 & 0.269 & $(.13)$ \\
\hline 11 & -0.229 & (.13) & 13 & 0.333 & $(.22)$ & 16 & 0.203 & $(.18)$ \\
\hline 20 & -0.336 & (.24) & 20 & 0.299 & (.18) & 10 & 0.056 & $(.28)$ \\
\hline 10 & -0.464 & (.14) & 21 & 0.131 & $(.22)$ & 20 & -0.076 & $(.24)$ \\
\hline 21 & -0.513 & $(.22)$ & 10 & 0.099 & $(.22)$ & 21 & -0.258 & $(.23)$ \\
\hline 19 & -0.567 & $(.21)$ & 19 & 0.051 & (.18) & 19 & -0.290 & $(.21)$ \\
\hline 18 & -0.639 & (.13) & 18 & -0.521 & (.15) & 18 & -0.682 & $(.16)$ \\
\hline 28 & -1.091 & $(.12)$ & 28 & -1.968 & (.34) & 28 & -0.854 & $(.16)$ \\
\hline 26 & -1.315 & (.13) & 26 & -2.223 & (.35) & 26 & -1.087 & (.19) \\
\hline
\end{tabular}

Table 4.8 Comparison of part-worth estimates for the Toothpaste Study, sorted by relative size. 
Threshold model - with homogeneous scale use Column: many-to-one mapping

Unobserved heterogeneity - Posterior estimate of covariance matrix $V_{\beta}$

\begin{tabular}{|c|c|c|c|c|c|c|c|c|c|c|}
\hline & 1 & 2 & 3 & 4 & 5 & 6 & 7 & 8 & 9 & 10 \\
\hline 1 & 2.260 & & & & & & & & & \\
\hline 2 & 0.811 & 1.821 & & & & & & & & \\
\hline 3 & 0.370 & 0.486 & 2.106 & & & & & & & \\
\hline 4 & 0.376 & 0.325 & 0.049 & 1.492 & & & & & & \\
\hline 5 & 0.411 & 0.134 & 0.390 & 0.213 & 1.282 & & & & & \\
\hline 6 & -0.758 & -0.475 & -0.074 & 0.377 & 0.199 & 2.205 & & & & \\
\hline 7 & 0.542 & 0.419 & 0.066 & 0.453 & 0.181 & -0.208 & 1.789 & & & \\
\hline 8 & 0.331 & 0.462 & 0.050 & 0.501 & -0.050 & -0.089 & 0.941 & 1.736 & & \\
\hline 9 & -0.269 & -0.349 & 0.154 & -0.128 & 0.113 & 0.327 & 0.007 & -0.087 & 1.405 & \\
\hline 10 & -0.469 & -0.162 & -1.183 & -0.337 & -0.390 & 0.099 & 0.070 & 0.106 & -0.054 & 4.148 \\
\hline 11 & -0.451 & -0.231 & -0.286 & -0.209 & -0.527 & 0.171 & -0.279 & 0.132 & 0.190 & 1.038 \\
\hline 12 & -0.485 & -0.049 & -0.186 & -0.357 & -0.464 & 0.112 & -0.397 & -0.134 & -0.040 & 0.732 \\
\hline 13 & -0.457 & -0.320 & -0.796 & -0.141 & -0.559 & -0.095 & 0.056 & 0.206 & -0.275 & 1.454 \\
\hline 14 & -0.184 & -0.261 & -0.382 & -0.207 & 0.027 & 0.078 & -0.082 & -0.324 & 0.175 & 0.348 \\
\hline 15 & 0.711 & 0.602 & 0.183 & 0.545 & 0.187 & -0.305 & 0.489 & 0.594 & -0.352 & -0.806 \\
\hline 16 & -0.064 & 0.162 & 0.083 & 0.173 & -0.253 & 0.044 & 0.080 & 0.396 & -0.313 & -0.177 \\
\hline 17 & -0.055 & -0.149 & 0.245 & -0.170 & 0.301 & 0.065 & -0.284 & -0.479 & 0.297 & -0.449 \\
\hline 18 & -0.470 & -0.406 & 0.046 & -0.398 & 0.049 & 0.365 & -0.445 & -0.686 & 0.258 & 0.259 \\
\hline 19 & 0.359 & 0.234 & -0.237 & -0.135 & -0.057 & -0.248 & 0.122 & 0.007 & -0.034 & 0.658 \\
\hline 20 & -0.001 & 0.028 & -0.103 & -0.126 & 0.278 & -0.279 & 0.095 & -0.100 & 0.042 & 0.257 \\
\hline 21 & 0.623 & 0.656 & -0.040 & 0.031 & -0.145 & -0.498 & 0.305 & 0.309 & -0.183 & 0.340 \\
\hline 22 & 0.274 & 0.108 & 0.091 & 0.065 & 0.167 & -0.145 & 0.160 & 0.234 & -0.142 & -0.054 \\
\hline 23 & -0.060 & -0.434 & 0.250 & 0.265 & 0.291 & 0.380 & 0.197 & 0.138 & 0.082 & -0.328 \\
\hline 24 & 0.609 & 0.233 & 0.198 & 0.084 & 0.113 & -0.361 & 0.157 & 0.113 & 0.052 & -0.270 \\
\hline 25 & 0.227 & 0.443 & 0.107 & 0.075 & -0.178 & -0.368 & 0.183 & 0.407 & -0.313 & -0.036 \\
\hline 26 & -0.131 & -0.454 & 0.086 & -0.262 & 0.255 & 0.151 & -0.350 & -0.648 & 0.228 & -0.346 \\
\hline 27 & 0.317 & 0.258 & 0.376 & 0.244 & 0.190 & -0.120 & 0.139 & 0.227 & -0.006 & -0.670 \\
\hline 28 & -0.423 & -0.210 & -0.107 & -0.186 & -0.202 & 0.176 & -0.411 & -0.259 & -0.151 & 0.162 \\
\hline 29 & -0.375 & -0.308 & -0.405 & -0.094 & -0.270 & 0.189 & -0.201 & -0.150 & -0.063 & 0.409 \\
\hline 30 & -0.274 & -0.350 & -0.143 & -0.282 & 0.014 & 0.159 & -0.205 & -0.295 & 0.224 & 0.237 \\
\hline
\end{tabular}

Estimates in bold have more than $95 \%$ of posterior mass away from 0 .

Continued

Table 4.9 Posterior estimate of the covariance matrix for threshold variable selection model, column: many-to-one mapping with homogeneous scale use. 
Table 4.9 continued

\begin{tabular}{|c|c|c|c|c|c|c|c|c|c|c|}
\hline & 11 & 12 & 13 & 14 & 15 & 16 & 17 & 18 & 19 & 20 \\
\hline 1 & & & & & & & & & & \\
\hline 2 & & & & & & & & & & \\
\hline 3 & & & & & & & & & & \\
\hline 4 & & & & & & & & & & \\
\hline 5 & & & & & & & & & & \\
\hline 6 & & & & & & & & & & \\
\hline 7 & & & & & & & & & & \\
\hline 8 & & & & & & & & & & \\
\hline 9 & & & & & & & & & & \\
\hline 10 & & & & & & & & & & \\
\hline 11 & 2.484 & & & & & & & & & \\
\hline 12 & 0.628 & 1.880 & & & & & & & & \\
\hline 13 & 0.840 & 0.892 & 3.105 & & & & & & & \\
\hline 14 & -0.047 & 0.338 & 0.265 & 1.676 & & & & & & \\
\hline 15 & -0.343 & -0.517 & -0.914 & -0.217 & 2.380 & & & & & \\
\hline 16 & 0.523 & 0.032 & 0.173 & -0.391 & 0.765 & 1.664 & & & & \\
\hline 17 & -0.443 & -0.234 & -0.818 & 0.152 & 0.317 & -0.071 & 1.387 & & & \\
\hline 18 & -0.146 & 0.109 & -0.200 & 0.217 & -0.609 & -0.182 & 0.741 & 1.964 & & \\
\hline 19 & 0.060 & 0.056 & -0.036 & 0.316 & 0.120 & -0.251 & 0.058 & -0.085 & 1.785 & \\
\hline 20 & -0.322 & -0.378 & -0.113 & 0.251 & -0.081 & -0.228 & 0.129 & 0.094 & 0.385 & 1.936 \\
\hline 21 & 0.005 & 0.143 & 0.049 & -0.033 & 0.445 & -0.029 & -0.271 & -0.398 & 0.984 & 0.251 \\
\hline 22 & -0.088 & -0.260 & -0.313 & 0.199 & 0.355 & -0.142 & 0.129 & -0.184 & 0.408 & -0.010 \\
\hline 23 & -0.447 & -0.389 & -0.217 & -0.124 & -0.184 & 0.099 & 0.016 & 0.237 & -0.382 & 0.025 \\
\hline 24 & -0.452 & -0.179 & -0.322 & -0.097 & 0.154 & -0.358 & 0.161 & 0.032 & 0.261 & -0.051 \\
\hline 25 & 0.480 & 0.225 & 0.253 & -0.235 & 0.259 & 0.437 & -0.471 & -0.464 & -0.250 & -0.166 \\
\hline 26 & -0.143 & -0.145 & -0.359 & 0.155 & -0.270 & -0.269 & 0.377 & 0.495 & -0.099 & 0.223 \\
\hline 27 & -0.395 & -0.384 & -0.560 & -0.227 & 0.698 & 0.054 & 0.113 & -0.384 & -0.059 & -0.134 \\
\hline 28 & 0.328 & 0.205 & 0.162 & 0.008 & -0.263 & 0.147 & 0.009 & 0.199 & -0.077 & 0.035 \\
\hline 29 & 0.519 & 0.144 & 0.568 & -0.112 & -0.344 & 0.296 & -0.218 & 0.071 & -0.225 & -0.111 \\
\hline 30 & 0.200 & 0.127 & 0.088 & 0.256 & -0.396 & -0.302 & 0.165 & 0.209 & 0.088 & -0.131 \\
\hline
\end{tabular}

Estimates in bold have more than $95 \%$ of posterior mass away from 0 .

Continued 
Table 4.9 continued

\begin{tabular}{|c|c|c|c|c|c|c|c|c|c|c|}
\hline & 21 & 22 & 23 & 24 & 25 & 26 & 27 & 28 & 29 & 30 \\
\hline 1 & & & & & & & & & & \\
\hline 2 & & & & & & & & & & \\
\hline 3 & & & & & & & & & & \\
\hline 4 & & & & & & & & & & \\
\hline 5 & & & & & & & & & & \\
\hline 6 & & & & & & & & & & \\
\hline 7 & & & & & & & & & & \\
\hline 8 & & & & & & & & & & \\
\hline 9 & & & & & & & & & & \\
\hline 10 & & & & & & & & & & \\
\hline 11 & & & & & & & & & & \\
\hline 12 & & & & & & & & & & \\
\hline 13 & & & & & & & & & & \\
\hline 14 & & & & & & & & & & \\
\hline 15 & & & & & & & & & & \\
\hline 16 & & & & & & & & & & \\
\hline 17 & & & & & & & & & & \\
\hline 18 & & & & & & & & & & \\
\hline 19 & & & & & & & & & & \\
\hline 20 & & & & & & & & & & \\
\hline 21 & 1.927 & & & & & & & & & \\
\hline 22 & 0.218 & 2.048 & & & & & & & & \\
\hline 23 & -0.592 & 0.072 & 2.816 & & & & & & & \\
\hline 24 & 0.270 & 0.491 & 0.493 & 1.451 & & & & & & \\
\hline 25 & 0.113 & 0.116 & -0.292 & -0.099 & 2.102 & & & & & \\
\hline 26 & -0.267 & -0.179 & 0.155 & -0.078 & -0.275 & 1.784 & & & & \\
\hline 27 & 0.133 & 0.127 & 0.038 & 0.235 & 0.116 & -0.030 & 1.809 & & & \\
\hline 28 & -0.157 & -0.214 & -0.146 & -0.269 & -0.015 & 0.879 & -0.218 & 1.605 & & \\
\hline 29 & -0.258 & -0.340 & -0.144 & -0.422 & 0.045 & 0.030 & 0.043 & 0.331 & 1.701 & \\
\hline 30 & -0.259 & 0.032 & -0.145 & -0.110 & 0.478 & 0.167 & -0.233 & 0.169 & 0.213 & 1.819 \\
\hline
\end{tabular}

Estimates in bold have more than $95 \%$ of posterior mass away from 0 . 
Threshold model - with homogeneous scale use

Column: many-to-one mapping

Estimates of $\theta$ : Average mapping between Concerns/Interests and Attributes/Benefits

\begin{tabular}{|c|c|c|c|c|c|}
\hline \multirow{2}{*}{\multicolumn{2}{|c|}{ Concern/Interest }} & \multicolumn{2}{|c|}{ Diagonal elements } & \multicolumn{2}{|c|}{ Off-Diagonal elements } \\
\hline & & \multirow{2}{*}{$\begin{array}{c}\text { Posterior } \\
\text { Mean } \\
0.340\end{array}$} & \multirow{2}{*}{$\begin{array}{c}\text { Posterior } \\
\text { Std. Dev. } \\
(.14)\end{array}$} & \multirow{2}{*}{$\begin{array}{c}\text { Posterior } \\
\text { Mean }\end{array}$} & \multirow{2}{*}{$\begin{array}{c}\text { Posterior } \\
\text { Std. Dev. } \\
(.14)\end{array}$} \\
\hline 1 & I am predisposed to having cavities & & & & \\
\hline 2 & I am concerned about not getting to hard to reach places & 0.275 & $(.14)$ & 0.378 & $(.16)$ \\
\hline 3 & I am concerned about tartar and plaque build-up on my teeth & 0.402 & $(.12)$ & 0.319 & $(.14)$ \\
\hline 4 & I am concerned about the condition of my gums & 0.307 & $(.14)$ & 0.363 & $(.15)$ \\
\hline 5 & I am concerned there are cavity prone places on my teeth & 0.272 & $(.12)$ & 0.385 & $(.15)$ \\
\hline 6 & I am concerned about germs and mouth infections & 0.350 & $(.15)$ & 0.317 & $(.14)$ \\
\hline 7 & Toothpastes are too strong tasting & 0.437 & $(.16)$ & 0.285 & $(.14)$ \\
\hline 8 & I enjoy the fresh taste I get from brushing & 0.329 & $(.11)$ & 0.400 & $(.14)$ \\
\hline 9 & I like the tingle I feel in my mouth after I brush & 0.144 & $(.07)$ & 0.520 & $(.16)$ \\
\hline 10 & I have trouble getting my kids to brush & 0.325 & $(.15)$ & 0.371 & $(.16)$ \\
\hline 11 & Bubbling action adds to the sensory pleasure of brushing & 0.243 & $(.11)$ & 0.365 & $(.15)$ \\
\hline 12 & Toothpastes irritate my mouth & 0.320 & $(.15)$ & 0.347 & $(.15)$ \\
\hline 13 & I am predisposed to having sensitive teeth & 0.395 & $(.15)$ & 0.297 & $(.14)$ \\
\hline 14 & Toothpastes scratch the enamel on my teeth & 0.348 & $(.15)$ & 0.324 & $(.14)$ \\
\hline 15 & Toothpastes aren't strong enough to prevent cavities & 0.297 & $(.15)$ & 0.369 & $(.16)$ \\
\hline 16 & My teeth stain easily & 0.354 & $(.15)$ & 0.316 & $(.14)$ \\
\hline 17 & My teeth are dull/not white enough & 0.300 & $(.14)$ & 0.365 & $(.15)$ \\
\hline 18 & I love to see my teeth gleaming like pearls & 0.318 & $(.15)$ & 0.382 & $(.16)$ \\
\hline 19 & I am concerned about bad breath & 0.246 & $(.12)$ & 0.418 & $(.16)$ \\
\hline & Toothpaste breath-freshening doesn't last long enough & 0.274 & $(.14)$ & 0.386 & $(.16)$ \\
\hline & I wake up with bad taste/feeling in my mouth & 0.297 & $(.14)$ & 0.372 & $(.16)$ \\
\hline & Toothpastes cost too much & 0.448 & $(.15)$ & 0.283 & $(.14)$ \\
\hline & Toothpastes contain artificial ingredients & 0.307 & $(.14)$ & 0.361 & $(.15)$ \\
\hline 24 & Toothpaste packaging can be harmful to the environment & 0.358 & $(.14)$ & 0.342 & $(.15)$ \\
\hline & $\begin{array}{l}\text { I don't have problems, worries or interests regarding my teeth. I just } \\
\text { brush my teeth regularly }\end{array}$ & 0.350 & $(.13)$ & 0.351 & $(.14)$ \\
\hline & $\begin{array}{l}\text { I like to try different teeth brushing techniques/routines just for a } \\
\text { change of pace }\end{array}$ & 0.321 & $(.14)$ & 0.328 & $(.15)$ \\
\hline & I would feel I'm letting myself down if I didn't brush regularly & 0.496 & $(.14)$ & 0.267 & $(.13)$ \\
\hline & $\begin{array}{l}\text { I'm interested in knowing about the science of oral hygiene - including } \\
\text { different kinds of brushes and toothpastes }\end{array}$ & 0.294 & $(.13)$ & 0.356 & $(.14)$ \\
\hline 29 & I believe that people expect me to brush regularly & 0.282 & $(.13)$ & 0.404 & $(.16)$ \\
\hline & $\begin{array}{l}\text { For me, brushing my teeth is just something I do with little thought or } \\
\text { interest. }\end{array}$ & 0.437 & $(.15)$ & 0.317 & $(.15)$ \\
\hline & Toothpastes claim more than they can deliver & 0.007 & $()$. & 0.375 & $(.15)$ \\
\hline
\end{tabular}

Table 4.10 Estimates of $\theta$ : Average mapping between concerns/interests and attributes/benefits for threshold selection model, column: many-to-one mapping, with homogeneous scale use. 


\section{Threshold model - with homogeneous scale use \\ Column: many-to-one mapping}

\section{Summary of attributes used: sorted by posterior mean}

\begin{tabular}{|clcc|}
\multicolumn{1}{l}{ Attribute/Benefit } & $\begin{array}{c}\text { Posterior } \\
\text { Mean }\end{array}$ & $\begin{array}{c}\text { Posterior } \\
\text { Std. Dev }\end{array}$ \\
\hline 27 & Helps you feel good about yourself for brushing regularly & 0.411 & $(.08)$ \\
\hline 8 & Fresh tasting & 0.348 & $(.08)$ \\
\hline 3 & Helps remove tartar \& plaque & 0.317 & $(.06)$ \\
\hline 18 & Makes your teeth gleam like pearls & 0.294 & $(.08)$ \\
\hline 29 & Shows others you care about your teeth & 0.269 & $(.07)$ \\
\hline 19 & Fights bad breath & 0.256 & $(.07)$ \\
\hline 30 & For everyday brushing & 0.246 & $(.04)$ \\
\hline 4 & Helps promote healthy gums & 0.241 & $(.06)$ \\
\hline 20 & Freshens breath for 12 hours & 0.217 & $(.05)$ \\
\hline 6 & Helps fight germs \& infections in your mouth & 0.215 & $(.04)$ \\
\hline 17 & Whitens your teeth & 0.215 & $(.05)$ \\
\hline 25 & For routine maintenance & 0.212 & $(.04)$ \\
\hline 21 & Helps take away morning breath & 0.209 & $(.04)$ \\
\hline 13 & For sensitive teeth & 0.205 & $(.04)$ \\
\hline 2 & Delivers protection in hard to reach places & 0.205 & $(.05)$ \\
\hline 22 & $20 \%$ less than regular price & 0.204 & $(.03)$ \\
\hline 1 & Helps prevent cavities & 0.198 & $(.04)$ \\
\hline 28 & An interesting way to clean teeth & 0.198 & $(.04)$ \\
\hline 11 & Great bubbling action & 0.193 & $(.04)$ \\
\hline 5 & Penetrates to strengthen your teeth against cavities & 0.185 & $(.03)$ \\
\hline 9 & Gives your mouth a tingle & 0.182 & $(.04)$ \\
\hline 15 & Helps clean teeth. & 0.169 & $(.03)$ \\
\hline 24 & $100 \%$ recyclable packaging & 0.167 & $(.03)$ \\
\hline 16 & Helps remove stains & 0.167 & $(.03)$ \\
\hline 7 & Mild tasting & 0.164 & $(.02)$ \\
\hline 26 & Provides a change of pace & 0.162 & $(.02)$ \\
\hline 23 & $100 \%$ natural ingredients & 0.161 & $(.03)$ \\
\hline 10 & A taste kid's love & 0.151 & $(.02)$ \\
\hline 14 & Safe for tooth enamel (non-scratching) & 0.133 & $(.02)$ \\
\hline 12 & Doesn't irritate your mouth & 0.133 & $(.02)$ \\
\hline & & & \\
\hline
\end{tabular}

Table 4.11 Summary of attributes used, sorted by posterior mean for the threshold variable selection model, column: many-to-one mapping, with homogeneous scale use. 
Threshold model - with homogeneous scale use

Column: many-to-one mapping

\section{Summary of unmet concerns/interests: sorted by posterior mean}

Concern/Interest

\begin{tabular}{|c|c|c|c|}
\hline 31 & Toothpastes claim more than they can deliver & 0.261 & $(.06)$ \\
\hline 19 & I am concerned about bad breath & 0.222 & $(.09)$ \\
\hline 8 & I enjoy the fresh taste I get from brushing & 0.217 & $(.11)$ \\
\hline 9 & I like the tingle I feel in my mouth after I brush & 0.207 & $(.09)$ \\
\hline 29 & I believe that people expect me to brush regularly & 0.196 & $(.09)$ \\
\hline 18 & I love to see my teeth gleaming like pearls & 0.193 & $(.09)$ \\
\hline 3 & I am concerned about tartar and plaque build-up on my teeth & 0.158 & $(.07)$ \\
\hline 27 & I would feel I'm letting myself down if I didn't brush regularly & 0.158 & $(.08)$ \\
\hline 4 & I am concerned about the condition of my gums & 0.151 & $(.07)$ \\
\hline 20 & Toothpaste breath-freshening doesn't last long enough & 0.145 & $(.06)$ \\
\hline 11 & Bubbling action adds to the sensory pleasure of brushing & 0.142 & $(.06)$ \\
\hline 2 & I am concerned about not getting to hard to reach places & 0.129 & $(.06)$ \\
\hline 17 & My teeth are dull/not white enough & 0.126 & $(.06)$ \\
\hline 21 & I wake up with bad taste/feeling in my mouth & 0.116 & $(.06)$ \\
\hline 6 & I am concerned about germs and mouth infections & 0.108 & $(.05)$ \\
\hline 28 & $\begin{array}{l}\text { I'm interested in knowing about the science of oral hygiene - including } \\
\text { different kinds of brushes and toothpastes }\end{array}$ & 0.107 & $(.05)$ \\
\hline 5 & I am concerned there are cavity prone places on my teeth & 0.094 & $(.04)$ \\
\hline 25 & $\begin{array}{l}\text { I don't have problems, worries or interests regarding my teeth. I just } \\
\text { brush my teeth regularly }\end{array}$ & 0.083 & $(.04)$ \\
\hline 1 & I am predisposed to having cavities & 0.077 & $(.04)$ \\
\hline 13 & I am predisposed to having sensitive teeth & 0.076 & $(.03)$ \\
\hline 30 & $\begin{array}{l}\text { For me, brushing my teeth is just something I do with little thought or } \\
\text { interest. }\end{array}$ & 0.072 & $(.04)$ \\
\hline 15 & Toothpastes aren't strong enough to prevent cavities & 0.063 & $(.03)$ \\
\hline 26 & $\begin{array}{l}\text { I like to try different teeth brushing techniques/routines just for a } \\
\text { change of pace }\end{array}$ & 0.053 & $(.02)$ \\
\hline 22 & Toothpastes cost too much & 0.052 & $(.03)$ \\
\hline 23 & Toothpastes contain artificial ingredients & 0.050 & $(.02)$ \\
\hline 16 & My teeth stain easily & 0.049 & $(.02)$ \\
\hline 24 & Toothpaste packaging can be harmful to the environment & 0.044 & $(.02)$ \\
\hline 10 & I have trouble getting my kids to brush & 0.035 & $(.02)$ \\
\hline & Toothpastes are too strong tasting & 0.025 & $(.01)$ \\
\hline & Toothpastes irritate my mouth & 0.011 & $(.01)$ \\
\hline 14 & Toothpastes scratch the enamel on my teeth & 0.009 & $(.01)$ \\
\hline
\end{tabular}

Table 4.12 Summary of unmet concerns/interests, sorted by posterior mean for the threshold variable selection model, column: many-to-one mapping, with homogeneous scale use. 


\section{CHAPTER 5}

\section{CONCLUSIONS}

As Allenby and Rossi (p. 57, 1999) state, "The purpose of marketing is to understand consumer preferences and to help design and deliver appropriate goods and services." By understanding the diversity in consumer preferences marketers can offer differentiated products that better serve a segment of the market. This thesis provides improved methods for measuring and accounting for the diversity in consumer preferences.

Marketing researchers have procedural and statistical methods of obtaining measures of attribute importance for each individual on each attribute. In laboratory or experimental choice settings, studies can be designed to help focus respondents' attention and processing of the attributes. Statistical methods of modeling heterogeneity shrink poorly measured individual level parameters to the overall or group level mean.

However, it is erroneous to assume that consumers use all the product attributes in all brand choice situations. This thesis demonstrates that improved inference and predictive accuracy can be obtained by modeling which attributes are actually being used by consumers in different discrete choice situations.

This thesis contributes new models for determining at the individual level which product attributes are being used by a consumer in a brand choice decision. The 
heterogeneous variable selection model extends current aggregate level models of Bayesian variable selection. This model assumes a distribution of heterogeneity with mass concentrated at 0 and away from 0 . The pooled variable selection model allows the set of variables used by an individual to vary by choice context. Examples of separate contexts include partial and full profile choice experiments or choice experiments and actual market place transactions. A hybrid model combines the heterogeneous and pooled variable selection models. The threshold variable selection model incorporates insights from an extended model of choice and provides a behavioral explanation of why certain product attributes are used.

Tractable algorithms are introduced for estimating the proposed variable selection models. MCMC methods explore the posterior space of included variables and parameter estimates. Estimating variable selection models at the individual level presents challenges not encountered in the aggregate level models discussed in the statistics literature. Specifically, the proposed algorithms obtain meaningful distributions of heterogeneity and avoid the evaluation of complex marginal posterior distributions. Simulation and empirical applications demonstrate the efficacy of the models and the algorithms.

Two empirical studies are presented. In the Medical Device Study, the heterogeneous variable selection model, pooled variable selection model, and the hybrid model are fit to the data. In the Toothpaste Study, the heterogeneous, pooled, and threshold variable selection models are estimated. In both studies, a variable selection model fits the data better than baseline models with no variable selection and conventional distributions of heterogeneity. The Medical Device Study has data 
available for hold-out prediction and the variable selection models offer a 7 to $16 \%$ improvement in predictive accuracy. In the Toothpaste Study, the threshold variable selection model fit the data the best. Incorporating a behavioral explanation by including concerns and interests and a mapping between these and the product attributes improved the explanatory power of the model. In both studies, models that incorporate a qualitative shift between separate choice tasks did better than the heterogeneous variable selection model that assumes consumers use the same subset of attributes across tasks.

The variable selection models provide improved managerial insights. The Medical Device Study shows that ignoring variable selection leads to biased parameter estimates and different conclusions about the importance of individual product attributes. These differences would result in different optimal product designs. The Toothpaste Study highlights the usefulness of using an extended model of choice. The threshold variable selection model identifies specific concerns and interests among consumers that are not being met by current product offerings. These unmet concerns and interests represent opportunities to reposition specific brands and/or develop new product offerings.

There are several opportunities for extending the empirical applications. First, each of these studies included a change in context moving from partial to full profile choice situations. The heterogeneous variable selection model may perform better in data sets consisting of only full profile choice tasks. The heterogeneous or pooled variable selection models can be extended by adding another layer to the hierarchical model and estimating a statistical relationship between the variables selected and explanatory variables such as demographics, situation specific variables, etc. Third, since only one 
actual brand choice with measured concerns and interests was available in the Toothpaste Study, the full threshold variable selection model could not be estimated. A longitudinal study that records concerns and interests and brand choices across multiple purchase or choice occasions would provide the opportunity to estimate the full model. Fourth, it would be interesting to consider policy experiments that might result in changes to $\Lambda_{\mathrm{h}}$, the mapping between concerns and interests and product attributes. So, for instance, can promotions or advertising campaigns connect product attributes to previously unmet concerns and interests.

There are also opportunities to extend and/or refine technical aspects of the models. First, when studies rely on perceptual data this may introduce collinearity and complicate the variable selection problem. For the threshold variable selection model, correlated perceptions of product attributes may favor the column restricted, one-to-many mapping. Conversely, correlated measures of concerns and interests may favor the row restricted, many-to-one mapping. Second, although the discrepancy between the best fitting model and the best predicting model in the Medical Device Study was due to model averaging, it raises interesting questions about what exactly constitutes the "model" and how to choose the best "model." On theoretical grounds it should be resolved by the loss function. In some situations it may be "more costly" to retain or to continue to get measurements on all the variables of interest; in this case one would be led to choose a single representation of all possible combinations of variables from the posterior distribution. In other cases, all variables may be included in the final analysis by construction, but a flexible model for yielding the best predictive results may be optimal. In the Medical Device Study, all 16 attributes are going to be included in the 
final product design, the managerial question is what levels to choose for the attributes. This situation favors model averaging in simulation and predictive policy analysis.

There will always be opportunities for improving the algorithms proposed in this thesis. As more and more researchers adopt and use Bayesian and MCMC methods, improvements in general algorithms and methods will be discovered and documented. This progress will undoubtedly increase the accuracy and efficiency of existing models and expand the class of models that can be estimated. 
APPENDIX A

\section{DERIVATION OF CONDITION PROBABILITY} FOR THE HYBRID MODEL 
This appendix derives the conditional probability of $\tau_{\mathrm{hj}} \mid \lambda_{\mathrm{hj}}$ used in section 3.3.2, step 1 of the estimation algorithm for the hybrid model. Without loss of generality, the dependence on $\mathrm{h}$ and $\mathrm{j}$ will be dropped.

\section{Model Assumptions}

$$
\begin{aligned}
& \mathrm{p}(\tau=1)=\theta \\
& \mathrm{p}(\tau=0)=1-\theta \\
& \mathrm{p}(\lambda=1 \mid \tau=1)=\phi \\
& \mathrm{p}(\lambda=0 \mid \tau=1)=1-\phi \\
& \mathrm{p}(\lambda=1 \mid \tau=0)=0 \\
& \mathrm{p}(\lambda=0 \mid \tau=0)=1
\end{aligned}
$$

\section{Intermediate Quantities}

$$
\begin{array}{lll}
\mathrm{p}(\lambda=1) \quad \mathrm{p}(\lambda=1 \mid \tau=0) \times \mathrm{p}(\tau=0)+\mathrm{p}(\lambda=1 \mid \tau=1) \times \mathrm{p}(\tau=1) \\
\Rightarrow \quad(0 \times(1-\theta))+(\phi \times \theta)=(\phi \times \theta)
\end{array}
$$

by (A5), (A2), (A3), (A1), and the laws of conditional and total probability.

$$
\begin{array}{lll}
\mathrm{p}(\lambda=0) \quad & \mathrm{p}(\lambda=0 \mid \tau=0) \times \mathrm{p}(\tau=0)+\mathrm{p}(\lambda=0 \mid \tau=1) \times \mathrm{p}(\tau=1) \\
& \Rightarrow \quad(1 \times(1-\theta))+((1-\phi) \times \theta)=1-(\phi \times \theta)
\end{array}
$$

by (A6), (A2), (A4), (A1), and the laws of conditional and total probability. 


$$
\begin{aligned}
\mathrm{p}(\lambda=1 \cap \tau=1): & \mathrm{p}(\lambda=1 \mid \tau=1)=\frac{p(\lambda=1 \cap \tau=1)}{p(\tau=1)} \\
\Rightarrow & \phi=\frac{p(\lambda=1 \cap \tau=1)}{\theta} \\
\Rightarrow \quad & \mathrm{p}(\lambda=1 \cap \tau=1)=(\phi \times \theta)
\end{aligned}
$$

by law of conditional probability, (A3), and (A1).

$$
\begin{aligned}
& \mathrm{p}(\lambda=0 \cap \tau=1): \mathrm{p}(\lambda=0 \mid \tau=1)=\frac{p(\lambda=0 \cap \tau=1)}{p(\tau=1)} \\
& \Rightarrow \quad 1-\phi=\frac{p(\lambda=0 \cap \tau=1)}{\theta} \\
& \Rightarrow \quad \mathrm{p}(\lambda=0 \cap \tau=1)=((1-\phi) \times \theta)
\end{aligned}
$$

by law of conditional probability, (A4), and (A1).

\section{Quantities of Interest}

$$
\begin{aligned}
\mathrm{p}(\tau=1 \mid \lambda=1) & \Rightarrow \quad \frac{p(\lambda=1 \cap \tau=1)}{p(\lambda=1)} \\
& \Rightarrow \quad \frac{(\phi \times \theta)}{(\phi \times \theta)}=1
\end{aligned}
$$

by law of conditional probability, (A9), and (A7). 


$$
\begin{aligned}
\mathrm{p}(\tau=1 \mid \lambda=0) & \Rightarrow \quad \frac{p(\lambda=0 \cap \tau=1)}{p(\lambda=0)} \\
& \Rightarrow \frac{((1-\phi) \times \theta)}{(1-(\phi \times \theta))}
\end{aligned}
$$

by law of conditional probability, (A10), and (A8). 


\section{List of References}

Allenby, Greg M. and James L. Ginter (1995), "Using Extremes to Design Products and Segment Markets," Journal of Marketing Research, 32, 392-403.

Allenby, Greg M. and Peter E. Rossi (1999), "Marketing Models of Consumer Heterogeneity," Journal of Econometrics, 89, 57-78.

Arrora, Neeraj, Greg M. Allenby, and James L. Ginter (1998), "A Hierarchical Bayes Model of Primary and Secondary Demand," Marketing Science, Vol. 17, No. 1, 29-44.

Bagozzi, Richard P. (2000), "The Poverty of Economic Explanations of Consumption and an Action Theory Alternative," Managerial and Decision Economics, 21, 95109.

Bagozzi, Richard P. and Utpal Dholakia (1999), "Goal Setting and Goal Striving in Consumer Behavior," Journal of Marketing, 63, 19-32.

Bagozzi, Richard P., Zeynep Gurhan-Canli, and Joseph R. Priester (2002), The Social Psychology of Consumer Behavior. Philadelphia: Open University Press.

Ben-Akiva, Moshe, Daniel McFadden, Kenneth Train, Joan Walker, Chandra Bhat, Michel Bierlaire, Denis Bolduc, Axel Borsch-Supan, David Brownstone, David S. Bunch, Andrew Daly, Andre De Palma, Dinesh Gopinath, Anders Karlstrom, and Marcela A. Munizaga (2002) "Hybrid Choice Models: Progress and Challenges," Marketing Letters, 13, 163-175.

Ben-Akiva, Moshe, Daniel McFadden, Tommy Garling, Dinesh Gopinath, Joan Walker, Denis Bolduc, Axel Borsch-Supan, Philippe Delquie, Oleg Larichev, Taka Morikawa, Amalia Polydoropoulou, and Vithala Rao (1999) "Extended Framework for Modeling Choice Behavior," Marketing Letters, 10, 187-203.

Bettman, James (1979), An Information Processing Theory of Consumer Choice. Reading, MA: Addison-Wesley. 
Bhat, Chandra R., and Saul Castelar (2002), "A Unified Mixed Logit Framework for Modeling Revealed and Stated Preferences: Formulation and Application to Congestion Pricing Analysis in the San Francisco Bay Area," Transportation Research Part B, 36, 593-616.

Bickel, Peter J. and Kjell A. Doksum (1977), Mathematical Statistics. Oakland, CA: Holden-Day, Inc.

Brownstone, David, David S. Bunch, and Kenneth Train (2000), "Joint Mixed Logit Models of Stated and Revealed Preferences for Alternative-Fueled Vehicles," Transportation Research Part B, 34, 315-338.

Casella, George and Edward George (1992), "Explaining the Gibbs Sampler," The American Statistician, Vol. 46, No. 3, 167-174.

Chapman, Randall G. and Richard Staelin (1982), "Exploiting Rand Ordered Choice Set Data Within the Stochastic Utility Model," Journal of Marketing Research, 29, 288-301.

Chib, Siddhartha and Edward Greenberg (1995), "Understanding the Metropolis-Hastings Algorithm," The American Statistician, Vol. 49, No. 4, 327-335.

Dholakia, Uptal M., and Richard P. Bagozzi (2002), "Mustering Motivation to Enact Decisions: How Decision Process Characteristics Influence Goal Realization," Journal of Behavioral Decision Making, 15, 167-188.

Edwards, Yancy D. (2002), "Identifying Poorly Met Demand: The Impact of Product Beliefs on Attribute Importance," Unpublished Dissertation, Ohio State University.

Edwards, Yancy D. and Greg M. Allenby (2003), "Multivariate Analysis of Multiple Response Data," Journal of Marketing Research, 40, 321-334.

Engel, James F., David T. Kollat, and Roger D. Blackwell (1968). Consumer Behavior. New York: Holt, Rinehart and Winston.

Fennell, Geraldine (1978), "Consumers' Perceptions of the Product-Use Situation," Journal of Marketing, (April), 38-47.

Fennell, Geraldine (1980), "The Situation," Motivation \& Emotion, 4, (December), 299322.

Fennell, Geraldine (1985), "Persuasion: Marketing as Behavioral Science in Profit and Nonprofit Contexts," in R. Belk (Ed.). Advances in Nonprofit Marketing. Greenwich, CT: JAI. 
Fennell, Geraldine (1988), "Action as Counterchange: Identifying the Motivatings of the Domain and Goal of Action," in L. Alwitt (Ed.), Proceedings, Division 23, 95 Annual Convention of the American Psychological Association.

Fennell, Geraldine and Greg M. Allenby (2003), "Conceptualizing and Measuring User Wants: Understanding the Source of Brand Preference," Working Paper, Ohio State University.

Fishbein, M. and I. Ajzen (1975). Belief, Attitude, Intention, and Behavior: An Introduction to Theory and Research. Reading, MA: Addison-Wesley.

George, Edward I. (2000), "The Variable Selection Problem," Journal of the American Statistical Association, Vol. 95, No. 452, 1304 - 1308.

George, Edward I. and Robert E. McCulloch (1993), "Variable Selection Via Gibbs Sampling," Journal of the American Statistical Association, Vol. 88, No. 423, 881-889.

George, Edward I. and Robert E. McCulloch (1997), "Approaches for Bayesian Variable Selection," Statistica Sinica, 7, 339-373.

Geweke, J. (1996). "Variable Selection and Model Comparison in Regression," in Bayesian Statistics 5 (Edited by J. M. Bernardo, J. O. Berger, A. P. Dawid, and A. F. M. Smith), 609-620. Oxford Press.

Gilbride, Timothy J. and Greg M. Allenby (2003), "A Choice Model with Conjunctive, Disjunctive, and Compensatory Screening Rules", Marketing Science, forthcoming.

Green, P. J. (1995), "Reversible Jump Markov Chain Monte Carlo Computation and Bayesian Model Determination," Biometrika, 82, 711-732.

Hoeting, Jennifer A., David Madigan, Adrian E. Raftery, and Chris T. Volinsky (1999), "Bayesian Model Averaging: A Tutorial," (with discussion) Statistical Science, Vol. 14, No. 4, 382-417.

Howard, John A. and Jagdish N. Sheth (1969). The Theory of Buyer Behaivor. New York: Wiley.

Madigan, D. and A. E. Raftery (1994), "Model Selection and Accounting for Model Uncertainty in Graphical Methods Using Occam's Window," Journal of the American Statistical Association, Vol. 89, No. 428, 1535-1546.

McFadden, Daniel (1986), "The Choice Theory Approach to Market Research," Marketing Science, Vol. 5, No. 4, 275-297. 
McFadden, Daniel (1999), "Rationality for Economists?" Journal of Risk and Uncertainty, 19:1-3, 73-105.

McFadden, Daniel (2001), "Economic Choices," The American Economic Review, Vol. 91, No. 3, 351-378.

Morikawa, Taka, Moshe Ben-Akiva, and Daniel McFadden (2002), "Discrete Choice Models Incorporating Revealed Preferences and Psychometric Data," in Econometric Models in Marketing, Vol. 16, 29-55.

Newton, Michael A. and Adrian E. Raftery (1994), "Approximating Bayesian Inference with the Weighted Likelihood Bootstrap," Journal of the Royal Statistical Society (B), 56, 3-48.

Nicosia, F. (1966). Consumer Decision Processes: Marketing and Advertising Implications. Englewood Cliffs, NJ: Prentice-Hall.

Peruginin, Marco and Richard P. Bagozzi (2001), "The Role of Desires and Anticipated Emotions in Goal-Directed Behaviours: Broadening and Deepening the Theory of Planned Behaviour," British Journal of Social Psychology, 40, 79-98.

Perugini, Marco and Mark Conner (2000) "Predicting and Understanding Behavioral Volitions: The Interplay Between Goals and Behaviors," European Journal of Social Psychology, 30, 705-731.

Phillips, David B. and Adrian F. M. Smith (1995). "Bayesian Model Comparison Via Jump Diffusions," in Markov Chain Monte Carlo in Practice (Editors W. R. Gilks, S. Richardson, and D. J. Spiegelhalter), 215 - 239. London: Chapman \& Hall.

Raftery, Adrian E., David Madigan, and Jennifer A. Hoeting (1997), "Bayesian Model Averaging for Linear Regression Model," Journal of the American Statistical Association, Vol. 92, No. 437, 179 - 191.

Rossi, Peter E. and Greg M. Allenby (2003), "Bayesian Statistics and Marketing," Marketing Science, Vol. 22, No. 3, 304-328.

Tanner, Martin A. (1993), Tools for Statistical Inference. New York:Springer-Verlag.

Tranel, Daniel, Antione Bechara, and Antonio R. Damasio (2002), "Decision Making and the Somatic Marker Hypothesis," in Michael S. Gazzaniga (Ed.), The New Cognitive Neurosciences. Cambridge, MA: The MIT Press. 
Walker, Joan and Moshe Ben-Akiva (2002), "Generalized Random Utility Model," Working Paper, MIT.

Yang, Sha, Greg M. Allenby, and Geraldine Fennell (2002), "Modeling Variation in Brand Preference: The Roles of Objective Environment and Motivating Conditions," Marketing Science, Vol. 21, No. 1, 14-31.

Yang, Sha and Greg M. Allenby (2000) "A Model for Observational, Structural, and Household Heterogeneity in Panel Data," Marketing Letters, 11, 137-149. 